\title{
AN ANALYSIS OF THE ARC AND SPARK SPECTRA OF YTTRIUM (Yt I AND Yt II)
}

\author{
By William F. Meggers and Henry Norris Russell
}

\begin{abstract}
All the available data (wave-length measurements and intensity estimates, temperature classes, Zeeman effects) on yttrium lines have been correlated and interpretated in an analysis of the arc and spark spectra. The total number of lines classified is 448 in the Yt I spectrum, 223 in the Yt II spectrum, and 10 in the Yt III spectrum.

Series-forming terms have been identified in each of the spectra, and from these the ionization potentials of 6.5 volts for the neutral Yt atom, 12.3 volts for the $\mathrm{Yt}^{+}$atom, and 20.6 volts for the $\mathrm{Yt}^{++}$atom have been deduced.

Yttrium is a chemical analogue of scandium, and the corresponding spectra are now seen to be strikingly similar. A ${ }^{2} \mathrm{D}$ term (from a $d$ electron) represents the lowest energy (normal state) of both Sc III and Yt III, and another ${ }^{2} \mathrm{D}$ (from the $s^{2} d$ configuration) describes the normal state of Sc I and of Yt I. The configurations which do not contain a $d$-electron are much lower in Yt II than in Sc II; a consequence of the fact that the $s$ and $p$ states in Yt III are lower compared with the $d$ state than in Sc III. This results in ${ }^{1} \mathrm{~S}_{\odot}\left(s^{2}\right)$ as the normal state for Yt II, while it is ${ }^{3} \mathrm{D}(s d)$ for Sc II.

The analyses of Yt I and Yt II spectra are supported by measurements of Zeeman effects, which are interpreted with the aid of Landé's theory of Zeeman effects and multiplet structure. All details of the three spectra, Yt I, Yt II, and Yt III, are in accord with Hund's correlation of spectral terms with electron configurations.
\end{abstract}

\section{CONTENTS}

I. Introduction

II. The spectrum of doubly ionized yttrium (Yt III)

III. The spectrum of singly ionized yttrium (Yt II) _.

IV. The spectrum of neutral yttrium (Yt I)

V. Theoretical interpretation of the spark spectra and comparison with scandium

VI. A note on Sc II

VII. Interpretation of the terms of Yt I _

VIII. Ionization potentials of yttrium atoms

IX. Yttrium in the sun .

\section{INTRODUCTION}

Last year the authors ${ }^{1}$ published an analysis of the arc and spark spectra of scandium ( $\mathrm{Sc}, \mathrm{Z}=21$ ), the lightest element exhibiting a spectrum of the type characteristic of the heavier metals in which

1 Russell and Meggers, B. S. Sci. Paper, No. 558, 22, p. 329; 1927.

$28183^{\circ}-29-1$ 
the lines are very numerous, multiplets are abundant, and series are inconspicuous. Yttrium $(\mathrm{Yt}, \mathrm{Z}=39$ ) is a chemical analogue of scandium; it occupies a corresponding position in the second long period of elements, and, since it has the same number and type of outer electrons, its spectral structures may be expected to resemble those of scandium. Such resemblances between homologous spectra are often very close (as for $\mathrm{Ca}$ and $\mathrm{Sr}$, which immediately precede $\mathrm{Sc}$ and $\mathrm{Yt}$ ). In other instances, however, there are minor but significant differences (mainly in the relative levels of the terms) which may prove of importance in the refinement of present atomic theories.

In the present paper the authors give the results of an extensive analysis of yttrium spectra in much the same manner as their results for scandium; the summary of recent theory and, except for a slight variation, the notation given in the earlier publication apply also to the present case.

The first regularities in yttrium spectra were found in 1914 by Popow, ${ }^{2}$ who measured the Zeeman effects of certain lines and recognized two "compound triplets" of six lines each. These cover the intervals $3179-3242 \mathrm{~A}$ and $4199-4423 \mathrm{~A}$; both are of the type ${ }^{3} \mathrm{D}-{ }^{3} \mathrm{P}$ and arise from ionized yttrium atoms. A larger number of lines with wave-number differences of 205,405 , and $1,847 \mathrm{~cm}^{-1}$ was listed. by Paulson ${ }^{3}$ in 1915 . These differences express the separations of four low-energy levels in the $\mathrm{Yt}_{\mathrm{t}}$ II spectrum now identified as $a^{3} \mathrm{D}_{1}, a^{3} \mathrm{D}_{2}, a^{3} \mathrm{D}_{3}, a^{1} \mathrm{D}_{2}$. Additional multiplets belonging to the Yt II spectrum, and the first regularities among arc lines (compound doublets in the Yt I spectrum) were published by Meggers ${ }^{4}$ in 1924, and new observations of Zeeman effects enabled Meggers and Moore ${ }^{5}$ to find the principal quartet-system multiplets in Yt I. Further extensions of the analyses of both spectra were published in 1926 by Meggers and Kiess, ${ }^{6}$ who found singlet terms in Yt II and also intercombinations between the singlet and triplet terms of Yt II and between the doublet and quartet terms of Yt I.

The lowest terms then found were a doublet-D in $\mathrm{Yt} \mathrm{I}$ and a triplet-D term in Yt II (resembling the normal terms of scandium). The complete analysis shows that the former corresponds to the normal state of neutral yttrium, while the normal state of the ionized atom is given by a singlet-S term about 0.1 volt lower in the energy diagram than ${ }^{3} \mathrm{D}$. For the higher homologue, lanthanum, the normal states appear to correspond to ${ }^{2} \mathrm{D}$ for La $\mathrm{I}$ and to ${ }^{2} \mathrm{~F}^{\prime}$ for $\mathrm{La}$ II. These differences find a simple explanation on Hund's theory ${ }^{7}$ of spectral structure.

Popow, Ann. d. Physik, 45, p. 163; 1914.

${ }^{3}$ Paulson, Astrophys. J., 41, p. 72; 1915.

1 Meggers, J., Wash. Acad. Sci., 14, p. 427; 1924.

8 Meggers and Moore, J., Wash. Acad. Sci., 15, p. 207; 1925.

6 Meggers and Kiess, J., Opt. Soc. Am., 12, p. 417; 1926.

; Hund, Linienspektren und periodisches system der Elemente, Julius Springer, Berlin; 1927. 
The preliminary classifications of yttrium lines were seriously handicapped by the lack of descriptive data. Although tables of wave lengths and intensity estimates in arc and spark spectra have been published by many observers, the lists, individually and collectively, still left much to be desired in the way of completeness and consistency. For these reasons the arc and spark spectra of yttrium were recently remeasured by one of us. ${ }^{8}$ Many new lines were recorded in the infra-red where an interval of nearly $2000 \mathrm{~A}$ was added to the arc spectrum, and a critical differentiation of lines belonging, respectively, to the Yt I, Yt II, and Yt III spectra resulted from a comparison of line intensities in arc and spark sources. In addition to these new wave-length determinations the Zeeman effects for 220 lines were measured by one of us, ${ }^{9}$ and the excellent separation of 451 yttrium lines into classes corresponding to their development with furnace temperatures has been recently published by King and Carter. ${ }^{10}$ These new data have made it relatively easy to classify almost all the lines ascribed to yttrium atoms, to specify without ambiguity practically all of the spectral terms, and correlate them with electron configurations. It has been possible to establish and identify many high (third set) terms in both the Yt I and the Yt II spectra. In both spectra certain of these higher terms have been identified as the second members of series, the extrapolation of which lead to ionization potentials of 6.5 volts for neutral $\mathrm{Yt}$ atoms and 12.3 volts for $\mathrm{Yt}^{+}$atoms. The total number of classified lines in the various spectra is as follows: 448 in the $\mathrm{Yt} \mathrm{I} \mathrm{spectrum,} 223$ in the $\mathrm{Yt}$ II spectrum, and 10 in the Yt III spectrum.

The detailed results will be presented, first, for $\mathrm{Yt}$ III, then for Yt II, and finally for Yt I, thus proceeding from the relatively simple (alkali) case of 1 valence electron to the 2 -electron spectrum and lastly to the complex spectrum arising from the increased transition possibilities with the full complement of 3 electrons.

\section{THE SPECTRUM OF DOUBLY IONIZED YTTRIUM (Yt III)}

Yttrium belongs to the second long period in which electron orbits of the types $5 s, 5 p$, and $4 d$ are successively added to the completed krypton shell. In doubly ionized yttrium only one valency electron remains; in the normal state this electron is in a $4 d$ orbit, which produces a ${ }^{2} \mathrm{D}$ term. The next lowest state corresponds to the $5 s$ orbit, and higher states arise from $5 p, 6 s, 5 d$, and $4 f$ orbits. These terms and their combinations were published in 1926 by Bowen and Millikan, ${ }^{11}$ who gave 165,289 as the term value for ${ }^{2} D_{2}$. The terms

\footnotetext{
8 Meggers, B. S. Jour. Research, 1 (R P12), p. 319; September, 1928.

9 Meggers, B. S. Jour. Research, 1 (RP12), p. 319; September, 1928.

10 King and Carter, Astrophys. J., 65, p. 86; 1927.

11 Bowen and Millikan, Phys. Rev., 28, p. 923; 1926.
} 
measured upward from the lowest energy level are shown in Table 1 and the combinations in Table 2.

The lines of doubly ionized yttrium are characterized by an enormous intensity difference in spark and arc. Whereas the estimated intensities of these lines in the spark spectrum range from 20 to 200 only the four strongest lines involving the low ${ }^{2} \mathrm{D}$ and ${ }^{2} \mathrm{~S}$ terms have been observed in the 220-volt arc spectrum; these are 2367.25 (3), 2414.68 (5), 2817.03 (5), and 2945.92 (4).

The series limit 165,289 in the Yt III spectrum corresponds to an ionization potential of 20.4 volts for the $\mathrm{Yt}^{++}$atom.

TABLE 1.-Terms in the Yt III spectrum

\begin{tabular}{|c|c|c|c|}
\hline $\begin{array}{c}\text { Configu- } \\
\text { ration }\end{array}$ & Terms & \multicolumn{2}{|c|}{ Levels } \\
\hline $4 d$ & $\left\{\begin{array}{l}4^{2} \mathrm{D}_{2} \\
4^{2} \mathrm{D}_{3}\end{array}\right.$ & $\begin{array}{r}0.0 \\
7248\end{array}$ & 724.8 \\
\hline $5 s$ & $5^{2} \mathrm{~S}_{1}$ & $7,466.2$ & \\
\hline $5 p$ & $\begin{array}{l}5^{2} \mathrm{P}_{1} \\
5^{2} \mathrm{P}_{2}\end{array}$ & $\begin{array}{l}41,401.2 \\
42,954.7\end{array}$ & 1553.5 \\
\hline $6 s$ & $6{ }^{2} \mathrm{~S}_{1}$ & $86,713.9$ & \\
\hline $5 d$ & $5^{2} \mathrm{D}_{2}$ & $88,378.8$ & 198.3 \\
\hline $4 f$ & $4^{2} \mathrm{~F}_{3}$ & $101,090.0$ & \\
\hline
\end{tabular}

Table 2 gives the known lines of this spectrum. No additions have been made in the present paper, but the wave-length measurements and intensity estimates are new except for the first two lines.

TABLE 2.-Classified lines of Yt III

\begin{tabular}{|c|r|r|l|}
\hline$\lambda$ & Intensity & \multicolumn{1}{c|}{$\nu$ (vac) } & Terms \\
\cline { 1 - 3 } 1989.21 & 1 & $101,090.8$ & $4^{2} \mathrm{D}_{2}-4^{2} \mathrm{~F}_{3}$ \\
1996.37 & 2 & $100,364.3$ & $4^{2} \mathrm{D}_{3}-4^{2} \mathrm{~F}_{3}$ \\
$2,127.99$ & 100 & $46,977.8$ & $5^{2} \mathrm{P}_{1}-5^{2} \mathrm{D}_{2}$ \\
$2,191.22$ & 200 & $45,622.4$ & $5^{2} \mathrm{P}_{2}-5^{2} \mathrm{D}_{3}$ \\
$2,200.80$ & 50 & $45,423.8$ & $5^{2} \mathrm{P}_{2}-5^{2} \mathrm{D}_{2}$ \\
$2,206.22$ & 30 & $45,312.2$ & $5^{2} \mathrm{P}_{1}-6^{2} \mathrm{~S}_{1}$ \\
$2,284.5$ & 100 & $43,759.7$ & $5^{2} \mathrm{P}_{2}-6^{2} \mathrm{~S}_{1}$ \\
$2,327.30$ & 20 & $42,955.0$ & $4^{2} \mathrm{D}_{2}-5^{2} \mathrm{P}_{2}$ \\
$2,367.25$ & 200 & $42,230.2$ & $4^{2} \mathrm{D}_{3}-5^{2} \mathrm{P}_{2}$ \\
$2,414.68$ & 100 & $41,400.8$ & $4^{2} \mathrm{D}_{2}-52 \mathrm{P}_{1}$ \\
$2,817.03$ & 200 & $35,488.2$ & $5^{2} \mathrm{~S}_{1}-5^{2} \mathrm{P}_{2}$ \\
$2,945.92$ & 150 & $33,935.4$ & $5^{2} \mathrm{~S}_{1}-5^{2} \mathrm{P}_{1}$ \\
\hline
\end{tabular}

1 Quoted from Bowen and Millikan, Phys. Rev., 28, p. 923; 1926.

Of the remaining groups which might be anticipated in this spectrum 4D-6S should lie near $1000 \mathrm{~A}, 6 \mathrm{~S}-6 \mathrm{P}$ and $5 \mathrm{D}-4 \mathrm{~F}$ near $8000 \mathrm{~A}$, and $5 \mathrm{D}-6 \mathrm{P}$ beyond $9000 \mathrm{~A}$. In the visible region the combinations $6 \mathrm{P}-6 \mathrm{D}, 6 \mathrm{P}-7 \mathrm{~S}$ and $5 \mathrm{D}-5 \mathrm{~F}$ may be anticipated-the first two a little short of $5600 \mathrm{~A}$ and the last near $3800 \mathrm{~A}$-but all of these are probably very faint. Further extension of the spectrum is therefore likely to be difficult. 


\section{THE SPECTRUM OF SINGLY IONIZED YTTRIUM}

In addition to the relatively small number of lines characteristic of $\mathrm{Yt}^{++}$the spark spectrum of yttrium shows about 230 lines which are associated with $\mathrm{Yt}^{+}$. Comparison of arc and spark spectrograms readily identifies most of these lines and divides them roughly into two classes. The strongest lines arise from combinations of low energy states with the next higher or middle set; they are generated with almost equal intensity in a 6 -ampere, 220 -volt arc and in a highvoltage condensed discharge and have nearly the same appearance in both sources. The second class of lines is very much enhanced upon passing from the arc to the spark. In the latter source, they are often decidedly hazy and shaded toward longer wave lengths. In the arc they are faint, and many do not appear at all in this source. The majority of these lines lie in the ultra-violet region; practically all of them have been identified as combinations between the second or middle set of terms and a higher or third set. Their hazy and unsymmetrical character suggests that, as in other spectra, these high terms correspond to larger electron "orbits" and are thus especially susceptible to Stark- and "pressure" effects. In scandium such lines were recognized by their strong enchancement at the negative electrode of the arc. ${ }^{12}$

In general, the structure of the Yt II spectrum parallels that of Sc II. Most of the strong lines represent combinations between ${ }^{3} \mathrm{P}^{\prime},{ }^{3} \mathrm{D},{ }^{3} \mathrm{~F}^{\prime}$ terms of low energy and a triad ${ }^{3} \mathrm{P},{ }^{3} \mathrm{D}^{\prime},{ }^{3} \mathrm{~F}$ lying between 24,000 and $30,000 \mathrm{~cm}^{-1}$ higher. Two outstanding lines, 3327.89 (150 III E) and 4374.94 (300 III E), were described as singlet combinations by Meggers and Kiess, ${ }^{13}$ who also called attention to two prominent unclassified lines, 3496.08 (150 III E) and 3633.13 (300 III E), suspected of involving singlet terms. The first two of these arise from a ${ }^{1} \mathrm{D}$ term, analogous to one in Sc II. The other two were at first puzzling, but it was later found that they arise from a ${ }^{1} \mathrm{~S}$ term which lies $840.18 \mathrm{~cm}^{-1}$ below $a^{3} \mathrm{D}$ and defines the normal state of the ionized yttrium atom.

The singlet combination $a^{1} \mathrm{D}_{2}-z^{1} \mathrm{P}_{1}, 4127.57$ (2), is abnormally faint in Yt II. In Sc II it is considerably stronger, though still the faintest in the triad to which it belongs.

The terms which have been identified in the Yt II spectrum are listed in Table 3 . The term values are measured upward from the lowest known energy level, $a^{1} \mathrm{~S}_{0}$, which is undoubtedly the lowest in the-ionized atom. Term symbols, relative values of the levels, separations of the sublevels of polyfold terms, and combinations with other terms are given in successive columns.

12 Russell and Meggers, B. S. Sci. Papers No. 558, 22, p. 331; 1927.

${ }_{13}$ Meggers and Kiess, J. Opt. Soc. Am., 12, p. 425; 1926. 
All the terms are confirmed by combinations with two or more levels, except $b^{1} \mathrm{~S}_{0}$ and $b^{1} \mathrm{G}_{4}$, which depend on only a single line in each case. For the second of these the identification of the line, which is the strongest member of a "supermultiplet," is practically certain; for the first, it is probable, but not entirely assured.

TABLE 3.-Relative terms in the Yt II spectrum

\begin{tabular}{|c|c|c|c|c|c|c|c|}
\hline Term & Level & $\Delta \nu$ & Combinations & Term & Level & $\Delta \nu$ & Combinations \\
\hline$a^{1} \mathrm{~S}_{0}$ & 0.00 & & $z^{1} \mathrm{P}, y^{1} \mathrm{P}, z^{3} \mathrm{P}, y^{3} \mathrm{P}, z^{3} \mathrm{D}^{\prime}$ & $y^{1} \mathrm{P}_{1}$ & $44,568.2$ & & $a^{1} \mathrm{~S}, a^{1} \mathrm{D}, b^{1} \mathrm{D}, f^{1} \mathrm{D}, a^{3} \mathrm{P}^{\prime}$. \\
\hline$a^{5} \mathrm{D}_{1}$ & 840.18 & & & $b^{3} \mathrm{D}_{1}$ & $54,955.6$ & & \\
\hline$a^{3} \mathrm{D}_{2}$ & $1,045.09$ & $\begin{array}{l}204.91 \\
404.79\end{array}$ & 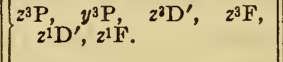 & $b^{3} \mathrm{D}_{2}$ & $55,032.0$ & $\begin{array}{r}76.4 \\
613.4\end{array}$ & $\begin{array}{l}z^{3} \mathrm{P}, y^{3} \mathrm{P}, z^{3} \mathrm{D}^{\prime}, z^{3} \mathrm{~F}, z^{1} \mathrm{P} \\
z^{1} \mathrm{D}^{\prime}, z^{1} \mathrm{~F} ?\end{array}$ \\
\hline$a^{3} \mathrm{D}_{3}$ & $1,449.88$ & & & $b^{3} \mathrm{D}_{3}$ & $55,645.4$ & & \\
\hline$a^{1} \mathrm{D}_{2}$ & $3,296.27$ & & $\begin{array}{l}z^{1} \mathrm{P}, y^{1} \mathrm{P}, z^{1} \mathrm{D}^{\prime}, z^{1} \mathrm{~F}, z^{3} \mathrm{P} \\
y^{3} \mathrm{P}, z^{3} \mathrm{D}^{\prime}, z^{3} \mathrm{~F}\end{array}$ & $c^{1} \mathrm{D}_{2}$ & $55,724.9$ & & ${ }^{11} \mathrm{P}, \quad z^{3} \mathrm{D}^{\prime}, \quad z^{1} \mathrm{~F}, \quad y^{3} \mathrm{P}$, \\
\hline$a^{3} \mathrm{~F}_{2}^{\prime}$ & $8,003.12$ & & & $a^{3} \mathrm{~S}_{1}$ & $58,261.7$ & & $z^{3} \mathrm{P}, y^{3} \mathrm{P}$ \\
\hline$a^{3} F_{3}^{\prime}$ & $8,328.01$ & $\begin{array}{l}324.89 \\
415.38\end{array}$ & $\left\{\begin{array}{l}y^{3} \mathrm{P}, z^{3} \mathrm{D}^{\prime}, z^{3} \mathrm{~F}, z^{1} \mathrm{P}, z^{1} \mathrm{D}^{\prime}, \\
z^{1} \mathrm{~F}\end{array}\right.$ & $a^{1} \mathrm{~F}_{3}^{\prime}$ & $58,533.0$ & & 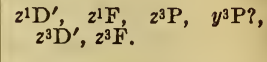 \\
\hline$a^{3} F_{1}^{\prime}$ & $8,743.39$ & & & $b^{3} P^{\prime} 0$ & $58,775.7$ & 371.5 & \\
\hline$a^{3} \mathrm{P}_{0}^{\prime}$ & $13,883.47$ & 134.86 & & & & 522.4 & $\begin{array}{l}z^{3} \mathrm{P}, \mathrm{y}, y^{3} \mathrm{P}, z^{3} \mathrm{D}^{\prime}, z^{3} \mathrm{~F} ?, \\
z^{1} \mathrm{D}^{\prime} .\end{array}$ \\
\hline$a^{3} \mathrm{P}_{1}^{\prime}$ & $14,018.33$ & 79.84 & zif. & $b^{3} \mathrm{P}_{2}^{\prime}$ & $59,069.6$ & & \\
\hline$a^{3} P_{2}^{\prime}$ & $14,098.17$ & & & $a^{3} \mathrm{G}_{3}$ & $59,178.9$ & 292.9 & \\
\hline$b^{1} D_{2}$ & $14,832.91$ & & $\begin{array}{l}z^{1} \mathrm{P}, y^{1} \mathrm{P}, z^{1} \mathrm{D}^{\prime}, z^{1} \mathrm{~F}, y^{3} \mathrm{P}, \\
z^{3} \mathrm{D}^{\prime}, z^{3} \mathrm{~F} .\end{array}$ & $\begin{array}{l}a^{3} \mathrm{G}_{4} \\
a^{3} \mathrm{G}_{5}\end{array}$ & $\begin{array}{l}59,471.8 \\
59,900.4\end{array}$ & 428.6 & $y^{3} \mathrm{P}, z^{3} \mathrm{D}^{\prime}, z^{3} \mathrm{~F}, z^{1} \mathrm{D}^{\prime}$ \\
\hline$a^{1} \mathrm{G}_{4}$ & $15,683.0$ & & $z^{1} \mathrm{~F}, z^{3} \mathrm{D}^{\prime}$ & $c^{3} \mathrm{D}_{1}$ & $58,719.7$ & & s \\
\hline $\begin{array}{l}2^{2} P_{0} \\
2^{3} P_{1}\end{array}$ & $\begin{array}{l}23,445.10 \\
23,776.26\end{array}$ & $\begin{array}{l}331.16 \\
870.92\end{array}$ & $\left\{\begin{array}{l}a^{3} \mathrm{~S}, b^{3} \mathrm{~S}, \quad b^{3} \mathrm{P}^{\prime}, \quad a^{3} \mathrm{D} \\
b^{3} \mathrm{D}, c^{3} \mathrm{D}, d^{3} \mathrm{D}, \quad a^{1} \mathrm{~S} \\
a^{1} \mathrm{D}, d^{1} \mathrm{D}, a^{1} \mathrm{~F}^{\prime} .\end{array}\right.$ & $\begin{array}{l}c^{3} \mathrm{D}_{2} \\
c^{3} \mathrm{D}_{3}\end{array}$ & $\begin{array}{l}58,946.6 \\
59,327.1\end{array}$ & $\begin{array}{l}226.9 \\
380.5\end{array}$ & $z^{3} \mathrm{P}, \quad z^{1} \mathrm{D}^{\prime}, z^{1} \mathrm{P}, \quad z^{3} \mathrm{D}^{\prime}, \quad z^{3} \mathrm{~F}$, \\
\hline $2^{3} \mathrm{P}_{3}$ & $24,647.18$ & & & & & & \\
\hline $2 ! D_{2}^{\prime}$ & $26,147.21$ & & $\begin{array}{r}a^{1} \mathrm{P}^{\prime}, a^{1} \mathrm{D}, \quad b^{1} \mathrm{D}, \quad c^{1} \mathrm{D} \\
d^{1} \mathrm{D}, e^{1} \mathrm{D}, f^{1} \mathrm{D}, a^{1} \mathrm{~F}^{\prime} \\
b^{3} \mathrm{P}^{\prime}, a^{3} \mathrm{D}, b^{3} \mathrm{D}, c^{3} \mathrm{D} \\
d^{3} \mathrm{D}^{\prime}, a^{3} \mathrm{~F}^{\prime}, b^{3} \mathrm{~F}^{\prime}, a^{3} \mathrm{G}\end{array}$ & $\begin{array}{l}a^{1} \mathrm{P}_{1}^{\prime} \\
d^{1} \mathrm{D}_{2}\end{array}$ & $\begin{array}{l}59,715.4 \\
60,535.1\end{array}$ & & $\begin{array}{l}z^{1} \mathrm{P}, z^{1} \mathrm{D}^{\prime}, z^{3} \mathrm{D}^{\prime}, z^{3} \mathrm{~F} . \\
z^{1} \mathrm{P}, z^{1} \mathrm{D}^{\prime}, z^{1} \mathrm{~F}, z^{3} \mathrm{P}, z^{3} \mathrm{~F}\end{array}$ \\
\hline${ }_{21} \mathrm{P}_{1}$ & $27,516.71$ & & $\begin{array}{l}a^{1 \mathrm{~S}}, b^{1} \mathrm{~S} ?, c^{1} \mathrm{~S}, a^{1} \mathrm{P}^{\prime}, a^{1} \mathrm{D}, \\
b^{1} \mathrm{D}, c^{1} \mathrm{D}, d^{1} \mathrm{D}, e^{1 \mathrm{D}}, \\
f^{1} \mathrm{D}, a^{3} \mathrm{P}^{\prime}, b^{3} \mathrm{P}^{\prime}, a^{3} \mathrm{D} \\
b^{3} \mathrm{D}, a^{3} \mathrm{~F}^{\prime},\left(b^{3} \mathrm{~F}^{\prime} ?\right) .\end{array}$ & $\begin{array}{l}b^{3} \mathrm{~S}_{1} \\
b^{3} \mathrm{~F}_{2}^{\prime} \\
b^{3} \mathrm{~F}_{3}^{\prime}\end{array}$ & $\begin{array}{l}61,200.2 \\
61,336.7 \\
61,650.5\end{array}$ & 313.8 & $\begin{array}{l}2^{3} \mathrm{P}, y^{3} \mathrm{P} \\
z^{3} \mathrm{D}^{\prime}, z^{3} \mathrm{~F},\left(z^{1} \mathrm{P} ? ?\right), z^{1} \mathrm{D}^{\prime} .\end{array}$ \\
\hline $2^{2} \mathrm{~F}_{2}$ & $27,226.97$ & 305.25 & 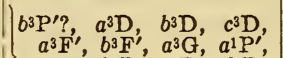 & $b^{3} \mathrm{~F}_{4}^{\prime}$ & $61,934.1$ & 283.6 & \\
\hline $2^{3} F_{4}$ & $\begin{array}{l}27,532.22 \\
28,394.31\end{array}$ & 862.09 & $\begin{array}{l}a^{1 \mathrm{D}}, b^{1} \mathrm{D}, c^{1} \mathrm{D}, d^{1} \mathrm{D} \\
e^{1} \mathrm{D}, a^{1} \mathrm{~F}^{\prime}\end{array}$ & $\begin{array}{l}c^{1} S_{0} \\
e^{1} D_{2}\end{array}$ & $\begin{array}{l}61,367.3 \\
62,495.2\end{array}$ & & $\begin{array}{l}z^{1} \mathrm{P}, z^{3} \mathrm{D}^{\prime} \\
z^{1} \mathrm{P}, z^{1} \mathrm{D}^{\prime}, z^{2} \mathrm{D}^{\prime}, z^{3} \mathrm{~F}\end{array}$ \\
\hline$z^{3} \mathrm{D}_{1}^{\prime}$ & $28,595.25$ & 134.80 & 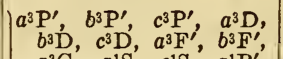 & $b^{1} G_{4}$ & $63,350.0$ & & $2^{1} \mathrm{~F}$ \\
\hline$z^{3} \mathrm{D}_{2}^{\prime}$ & $28,730.05$ & 484. 02 & 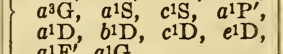 & $c^{3} P_{0}^{\prime}$ & $64,102.9$ & 159.8 & \\
\hline $2^{3} \mathrm{D}_{3}^{\prime}$ & $29,214.07$ & & $a^{1} \mathrm{~F}^{\prime}, a^{1} \mathrm{G}$ & $c^{3} \mathrm{P}_{1}^{\prime}$ & $64,262.7$ & & $y^{3} \mathrm{P}, 2^{3} \mathrm{D}^{\prime}$ \\
\hline$y^{2} \mathrm{P}_{0}$ & $32,048.82$ & 75.31 & $a^{3} \mathrm{~S}, b^{3} \mathrm{~S}, a^{3} \mathrm{P}^{\prime}, b^{3} \mathrm{P}^{\prime}, c^{3} \mathrm{P}^{\prime}$, & $c^{3} P_{2}^{\prime}$ & $64 ; 597.1$ & & \\
\hline$y^{3} \mathrm{P}_{1}$ & $32,124.13$ & 159.45 & a $a^{3} \mathrm{D}, \mathrm{F}^{3} \mathrm{D}, a^{3} \mathrm{G}, a^{1} \mathrm{~S}, a^{1} \mathrm{D}$, & $d^{3} \mathrm{D}_{1}$ & $65,131.5$ & 57.2 & \\
\hline$y^{3} \mathrm{P}_{2}$ & $32,283.58$ & & $b^{1} \mathrm{D}, c^{1} \mathrm{D}, a^{1} \mathrm{~F}^{\prime \prime} ?$ & $d^{3} \mathrm{D}^{2}$ & $65,188.7$ & & $z^{3} \mathrm{P}, y^{3} \mathrm{P}, 2^{1} \mathrm{D}^{\prime}$ \\
\hline $2^{1} \mathrm{~F}_{3}$ & $33,336.74$ & & $\begin{array}{l}a^{1} \mathrm{D}, b 1 \mathrm{D}, c^{1} \mathrm{D}, d^{1} \mathrm{D}, a^{1} \mathrm{~F}^{\prime} \\
a^{1} \mathrm{G}, b^{1} \mathrm{G}, a^{3} \mathrm{P}^{\prime}, a^{3} \mathrm{D} \\
b^{3} \mathrm{D} ?, c^{3} \mathrm{D}, a^{3} \mathrm{~F}^{\prime}\end{array}$ & $\begin{array}{l}d^{3} D_{3} \\
f^{1} D_{2}\end{array}$ & $\begin{array}{l}65,275.0 \\
70,223.2\end{array}$ & & $z^{1} \mathrm{P}, y^{1} \mathrm{P}, z^{1} \mathrm{D}^{\prime}$ \\
\hline
\end{tabular}

Diagrams of the terms and combinations are reproduced in Figures 1 and 2 , in which black dots represent singlet terms and circles triplet terms. Full lines represent singlet-singlet combinations, and 
triplet-triplet, and broken lines singlet-triplet combinations. The resemblance to the corresponding diagram ${ }^{14}$ for $\mathrm{Sc}$ II is striking.

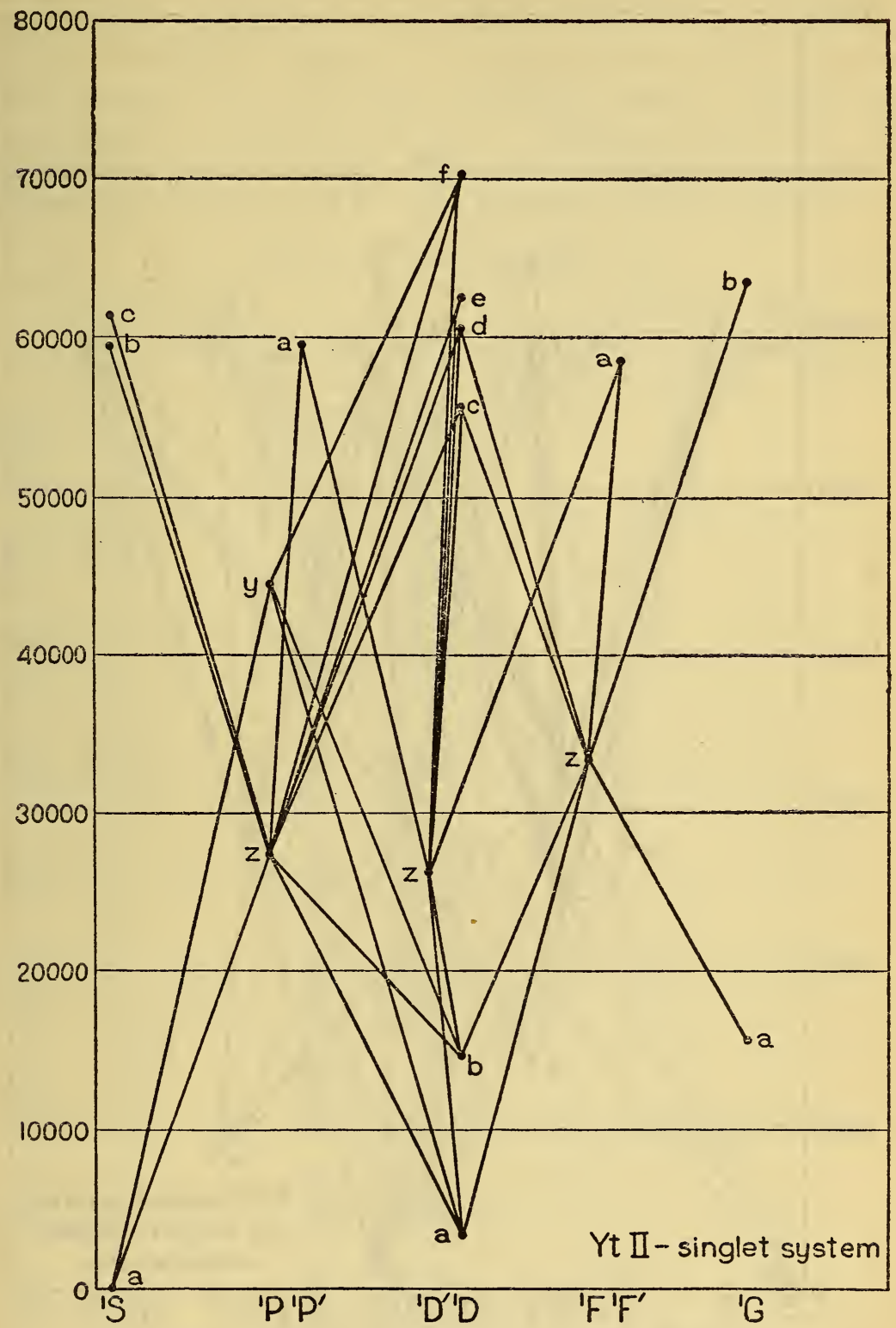

FIG. 1.-Energy diagram for the Yt II spectrum singlet system only See Figure 2 for triplet system and singlet-triplet combinations

The complete data for all classified lines of ionized yttrium are given in Table 4, successive columns of which contain the wave

14 Russell and Meggers, B. S. Sci. Papers No. 553, 3R, p. 334; 1927. 
lengths, arc and spark intensities, arc intensities and furnace classes by King and Carter (loc. cit.), the vacuum wave numbers, the ob-

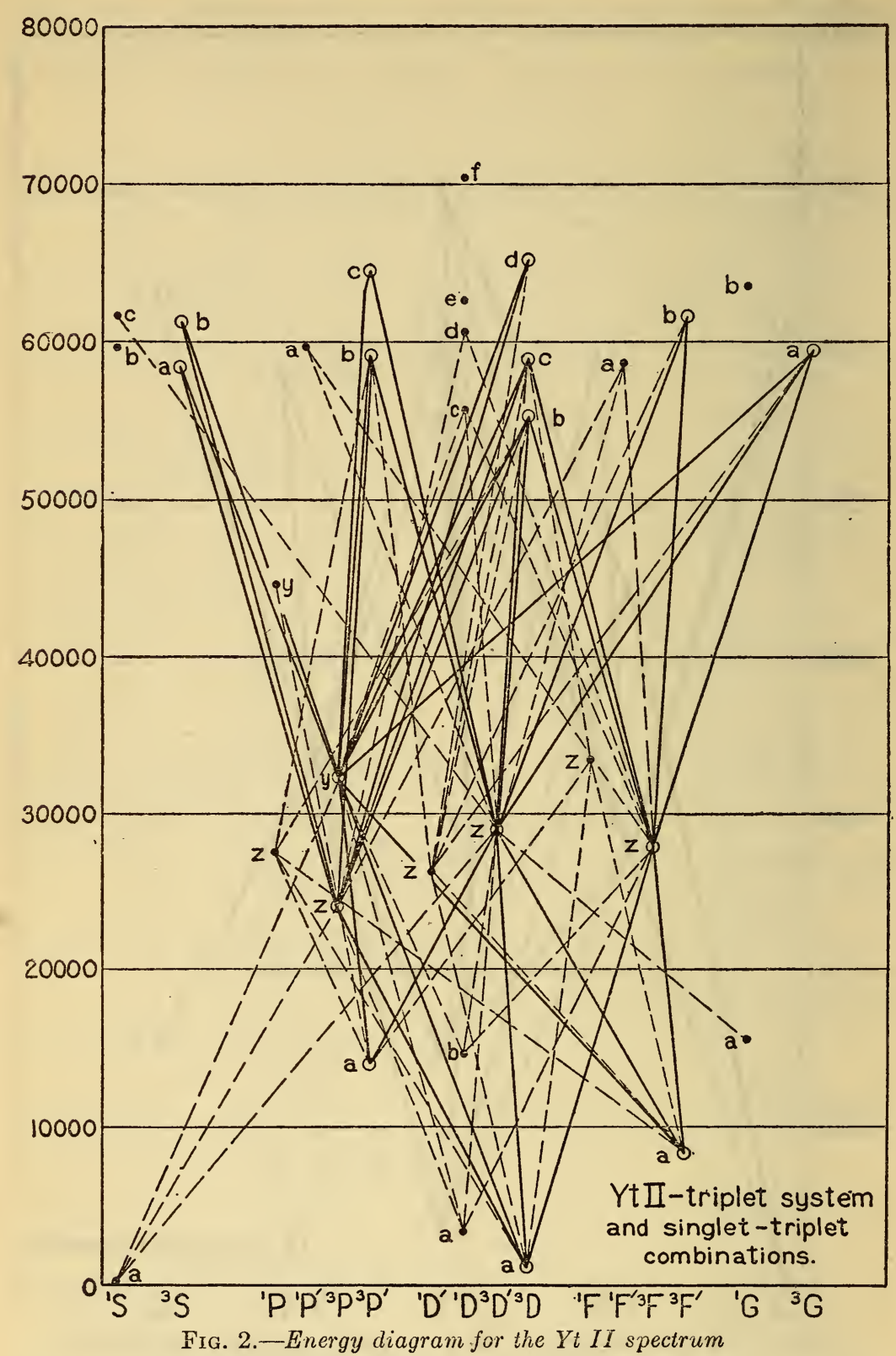

Black dots represent singlet terms, circles triplet terms. Full lines represent triplet-triplet combinations, and broken lines singlet-triplet combinations. See Figure 1 for singlet system

served and theoretical Zeeman effects, and finally the intensities on Rowland's scale of those lines which appear in the spectra of the 
sun's disk and of sun spots. The intensities and identifications of solar lines are taken from the Revision of Rowland's Table. ${ }^{15}$ Those in the sun-spot spectrum have been newly assigned by one of us (HNR) from examination of the Mount Wilson map of the spot spectra. The letter $\mathrm{b}$ following the intensity denotes that the yttrium line is blended in the sun with a fainter line, while $\mathrm{m}$ signifies that it is masked by a stronger solar line. $\mathrm{N}$ denotes a hazy line; ? that the identification is doubtful. The spot spectrum is unobservable to the violet of $3900 \mathrm{~A}$ and the solar spectrum ceases at $2975 \mathrm{~A}$.

TABLE 4.-Classified lines of Yt II

\begin{tabular}{|c|c|c|c|c|c|c|c|c|c|}
\hline \multirow{2}{*}{$\lambda$} & \multicolumn{2}{|c|}{$\begin{array}{c}\text { Intensity } \\
\mathbf{M}\end{array}$} & \multirow{2}{*}{$\begin{array}{c}\text { Intensity } \\
\text { Class K }\end{array}$} & \multirow{2}{*}{$\nu$} & \multirow{2}{*}{ Terms } & \multicolumn{2}{|c|}{ Zeeman effect } & \multicolumn{2}{|c|}{ Solar intensity } \\
\hline & Arc & Spark & & & & Observed & Calculated & Disk & Spot \\
\hline $\begin{array}{l}2,243.06 \\
2,268.14 \\
2,340.8 \\
2,398.14 \\
2,413.92\end{array}$ & $\begin{array}{l}\frac{25}{1} \\
1 \\
-\end{array}$ & $\begin{array}{l}50 \\
2 \mathrm{~h} \\
10 \mathrm{~h} \\
10 \mathrm{hl} \\
3 \mathrm{~h}\end{array} \mid$ & & $\begin{array}{l}44,568.1 \\
44,075.3 \\
42,707.3 \\
41,686.3 \\
41,413.8\end{array}$ & $\begin{array}{l}a^{1} \mathrm{~S}_{0}-y^{1} \mathrm{P}_{1} \\
z^{1} \mathrm{D}_{2}^{\prime}-f^{1} \mathrm{D}_{2} \\
z^{1} \mathrm{P}_{1}-f^{1} \mathrm{D}_{2} \\
z^{3} \mathrm{P}_{0}-d^{3} \mathrm{D}_{1} \\
z^{3} \mathrm{P}_{1}-d^{3} \mathrm{D}_{2}\end{array}$ & & -1 & & \\
\hline $\begin{array}{r}17.4 \\
22.22 \\
60.62 \\
2,465.90 \\
2,564.3\end{array}$ & $\begin{array}{r}1 \\
20 \\
2 \\
-\end{array}$ & $\begin{array}{l}5 \mathrm{~h} \\
50 \\
20 \\
5 \mathrm{~h} \\
1\end{array}$ & & $\begin{array}{r}354.2 \\
41,271.9 \\
40,627.9 \\
40,540.9 \\
38,985.3\end{array}$ & $\begin{array}{l}z^{3} \mathrm{P}_{1}-d^{3} \mathrm{D}_{1} \\
a^{1} \mathrm{D}_{2}-y^{1} \mathrm{P}_{1} \\
z^{3} \mathrm{P}_{2}-d^{3} \mathrm{D}_{3} \\
z^{3} \mathrm{P}_{2}-d^{3} \mathrm{D}_{2} \\
z^{1} \mathrm{D}_{2}^{\prime}-d^{3} \mathrm{D}_{1}\end{array}$ & & & & \\
\hline $\begin{array}{r}2,734.98 \\
50.40 \\
85.23 \\
2,785.60 \\
2,800.11\end{array}$ & $\begin{array}{l}z \\
z \\
z\end{array}$ & $\begin{array}{l}4 \mathrm{~h} \\
3 \mathrm{~h} \\
3 \\
2 \\
4\end{array}$ & & $\begin{array}{r}36,552.5 \\
36,347.6 \\
35,893.1 \\
888.3 \\
702.4\end{array}$ & $\begin{array}{l}z^{3} \mathrm{P}_{2}-b^{3} \mathrm{~S}_{1} \\
z^{1} \mathrm{D}_{2}^{\prime}-e^{1} \mathrm{D}_{2} \\
z^{3} \mathrm{P}_{1}-b^{3} \mathrm{P}^{\prime}{ }_{2} \\
z^{3} \mathrm{P}_{2}-d^{1} \mathrm{D}_{2} \\
z^{3} \mathrm{P}_{0}-b^{3} \mathrm{P}_{1}^{\prime}{ }_{1}\end{array}$ & & & & \\
\hline $\begin{array}{l}13.61 \\
25.37 \\
26.38 \\
34.57 \\
40.98\end{array}$ & $\frac{-}{\frac{1}{1}}$ & $\begin{array}{l}4 \mathrm{~h} \\
3 \mathrm{~h} \\
5 \\
5 \mathrm{~h} \\
5 \mathrm{~h}\end{array}$ & & $\begin{array}{l}531.1 \\
383.2 \\
370.6 \\
268.4 \\
188.8\end{array}$ & 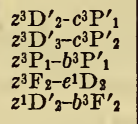 & & & & \\
\hline $\begin{array}{l}42.5 \\
54.45 \\
56.32 \\
58.06 \\
71.4\end{array}$ & $\begin{array}{l}-2 \\
1 \\
1 \\
-\end{array}$ & $\begin{array}{l}1 \\
15 \\
6 \\
4 \mathrm{~h} \\
1 \mathrm{~h}\end{array}$ & $\begin{array}{l}3 \mathrm{~V} \mathrm{E} \\
1 \mathrm{~V}\end{array}$ & $\begin{array}{r}170.0 \\
35,022.8 \\
34,999.8 \\
978.5 \\
816.0\end{array}$ & $\begin{array}{l}z^{3} \mathrm{P}_{1}-c^{3} \mathrm{D}_{2} \\
z^{3} \mathrm{P}_{2}-b^{3} \mathrm{P}^{\prime}{ }_{2} \\
z^{3} \mathrm{P}_{1}-b^{3} \mathrm{P}^{\prime}{ }_{0} \\
z^{1} \mathrm{P}_{1}-e^{1} \mathrm{D}_{2} \\
z^{3} \mathrm{P}_{0}-a^{3} \mathrm{~S}_{1}\end{array}$ & 1 & & & \\
\hline $\begin{array}{r}97.70 \\
2,898.93 \\
2,907.18 \\
30.15 \\
30.8\end{array}$ & $\frac{1}{1}$ & $\begin{array}{l}5 \\
3 \\
2 \\
6 \mathrm{~h} \\
2 \mathrm{~h}\end{array}$ & $\underset{\operatorname{tr} V}{1 \mathrm{~V}}$ & $\begin{array}{r}500.0 \\
485.4 \\
387.5 \\
118.0 \\
34,110.4\end{array}$ & $\begin{array}{l}z^{3} \mathrm{P}_{2}-b^{3} \mathrm{P}^{\prime}{ }_{1} \\
z^{3} \mathrm{P}_{1}-a^{3} \mathrm{~S}_{1} \\
z^{1} \mathrm{D}^{\prime}{ }_{2}-d^{1} \mathrm{D}_{2} \\
z^{3} \mathrm{~F}_{3}-b^{3} \mathrm{~F}^{\prime}{ }^{2} \\
z^{3} \mathrm{~F}_{2}-b^{3} \mathrm{~F}^{\prime}{ }_{2}\end{array}$ & & & f & \\
\hline $\begin{array}{l}48.98 \\
50.33 \\
53.28 \\
56.04 \\
57.39\end{array}$ & $\begin{array}{l}\bar{z} \\
\overline{1}\end{array}$ & $\begin{array}{l}3 \mathrm{~h} \\
1 \mathrm{~h} \\
3 \mathrm{~h} \\
5 \mathrm{~h} \\
2 \mathrm{~h}\end{array}$ & & $\begin{array}{r}33,900.1 \\
884.6 \\
850.8 \\
819.2 \\
803.8\end{array}$ & $\begin{array}{l}z^{3} \mathrm{D}_{1}^{\prime}{ }_{1}-e^{1} \mathrm{D}_{2} \\
z^{3} \mathrm{P}_{2}-a^{1} \mathrm{~F}^{\prime}{ }_{3} \\
z^{1} \mathrm{P}_{1}-c^{1} \mathrm{~S}_{0} \\
z^{1} \mathrm{P}_{1}-b^{3} \mathrm{~F}^{\prime}{ }^{2} \\
z^{3} \mathrm{~F}_{3}-b^{3} \mathrm{~F}^{\prime}{ }_{2}\end{array}$ & & 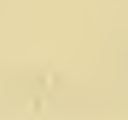 & . & \\
\hline $\begin{array}{r}74.02 \\
78.18 \\
80.7 \\
2,982.20 \\
3,001.42\end{array}$ & $\begin{array}{l}\frac{1}{2} \\
-\end{array}$ & $\begin{array}{l}5 \mathrm{~h} \\
3 \mathrm{~h} \\
20 \mathrm{hl} \\
2 \\
2\end{array}$ & $2 \mathrm{VE}$ & $\begin{array}{l}614.7 \\
567.8 \\
539.4 \\
522.5 \\
307.9\end{array}$ & $\begin{array}{l}z^{3} \mathrm{P}_{2}-a^{3} \mathrm{~S}_{1} \\
z^{1} \mathrm{D}_{2}^{\prime}{ }_{2}-a^{1} \mathrm{P}^{\prime}{ }_{1} \\
z^{3} \mathrm{~F}_{4}-b^{3} \bar{F}_{4}^{\prime} \\
z^{1} \mathrm{D}_{2}^{\prime}{ }_{2}-b^{3} \mathrm{P}^{\prime}{ }_{2} \\
z^{3} \mathrm{~F}_{2}-d^{1} \mathrm{D}_{2}\end{array}$ & & & & \\
\hline $\begin{array}{l}06.0 \\
23.50 \\
26.5 \\
27.75 \\
30.2\end{array}$ & $\frac{\frac{1}{2}}{\frac{1}{1}}$ & $\begin{array}{l}2 \mathrm{hl} \\
2 \mathrm{~h} \\
10 \mathrm{hl} \\
3 \\
4 \mathrm{~h}\end{array}$ & & $\begin{array}{r}257.1 \\
064.7 \\
031.9 \\
33,018.3 \\
32,991.5\end{array}$ & $\begin{array}{l}z^{3} \mathrm{~F}_{4}-b^{3} \mathrm{~F}^{\prime}{ }_{3} \\
y^{3} \mathrm{P}_{1}-d^{3} \mathrm{D}_{2} \\
z^{1} \mathrm{D}^{\prime}{ }_{2}-d^{3} \mathrm{G}_{3} \\
z^{1} \mathrm{P}_{1}-d^{1} \mathrm{D}_{2} \\
y^{3} \mathrm{P}_{2}-d^{3} \mathrm{D}_{3}\end{array}$ & & & & \\
\hline $\begin{array}{c}36.7 \\
50.5 \\
53.3 \\
55.3 \\
3,066.02\end{array}$ & $\begin{array}{r}3 \\
2 \\
4 \\
\end{array}$ & $\left|\begin{array}{l}25 \mathrm{hl} \\
1 \mathrm{~h} \\
15 \mathrm{hl} \\
50 \mathrm{hl} \\
4 \mathrm{~h}\end{array}\right|$ & & $\begin{array}{r}920.9 \\
772.0 \\
742.0 \\
720.5 \\
32,606.1\end{array}$ & $\begin{array}{l}z^{3} D^{\prime}{ }_{2}-b^{3} F^{\prime}{ }_{3} \\
z^{3} D_{1}^{\prime}-c^{1} S_{0} \\
z^{3} D_{1}^{\prime}-b^{3} F^{\prime}{ }_{2} \\
z^{3} D^{\prime}{ }_{3}-b^{3} F^{\prime}{ }_{4} \\
z^{3} D^{\prime}{ }_{2}-b^{3} F^{\prime}{ }_{2}\end{array}$ & & & & \\
\hline
\end{tabular}

is Papers of the Mount Wilson Observatory, 3, Washington; 1928. 
TABLE 4.-Classified lines of $Y t I I$-Continued

\begin{tabular}{|c|c|c|c|c|c|c|c|c|c|}
\hline \multirow{2}{*}{$\lambda$} & \multicolumn{2}{|c|}{$\begin{array}{c}\text { Intensity } \\
\mathrm{M}\end{array}$} & \multirow{2}{*}{$\begin{array}{c}\text { Intensity } \\
\text { Class K }\end{array}$} & \multirow{2}{*}{$\nu$} & \multirow{2}{*}{ Terms } & \multicolumn{2}{|c|}{ Zeeman effect } & \multicolumn{2}{|c|}{ Solar intensity } \\
\hline & Arc & Spark & & & & Observed & Calculated & Disk & Spot \\
\hline $\begin{array}{c}3,059.26 \\
77.14 \\
78.64 \\
81.6 \\
82.16\end{array}$ & $\begin{array}{l}\bar{z} \\
\bar{z}\end{array}$ & $\begin{array}{l}5 \mathrm{~h} \\
4 \mathrm{~h} \\
4 \mathrm{~h} \\
2 \mathrm{~h} \\
3 \mathrm{~h}\end{array}$ & $2 \mathrm{~V} \mathrm{E}$ & $\begin{array}{r}32,571.7 \\
488.3 \\
472.5 \\
441.3 \\
435.4\end{array}$ & $\begin{array}{l}z^{1} \mathrm{D}^{\prime}{ }_{2}-c^{3} \mathrm{D}_{1} \\
z^{3} \mathrm{~F}_{2}-a^{1} \mathrm{P}^{\prime}{ }_{1} \\
y^{3} \mathrm{P}_{1}-c^{3} \mathrm{P}_{1}^{\prime}{ }_{2} \\
2^{3} \mathrm{~F}_{2}-b^{3} \mathrm{P}^{\prime}{ }_{2} \\
z^{3} \mathrm{D}^{\prime}{ }_{3}-b^{3} \mathrm{~F}^{\prime}{ }_{3}\end{array}$ & & & & \\
\hline $\begin{array}{r}86.9 \\
93.76 \\
3,095.88 \\
3,103.3 \\
04.82\end{array}$ & $\begin{array}{l}5 \\
1 \\
3 \\
-\end{array}$ & $\begin{array}{l}30 \mathrm{hl} \\
10 \mathrm{~h} \\
5 \\
2 \mathrm{~h} \\
4 \mathrm{~h}\end{array}$ & $\begin{array}{l}7 \mathrm{~V} \\
25 \mathrm{VE}\end{array}$ & $\begin{array}{l}385.6 \\
313.8 \\
291.6 \\
214.4 \\
198.7\end{array}$ & $\begin{array}{l}2^{1} \mathrm{D}^{\prime}{ }_{2}-a^{1} \mathrm{~F}^{\prime} 3 \\
y^{3} \mathrm{P}_{2}-c^{3} \mathrm{P}^{\prime}{ }_{2} \\
a^{3} \mathrm{D}_{2}-z^{1} \mathrm{~F}_{3} \\
y^{3} \mathrm{P}_{0}-c^{3} \mathrm{P}^{\prime}{ }_{1} \\
z^{1} \mathrm{P}_{1}-a^{1} \mathrm{P}_{1}^{\prime}{ }_{1}\end{array}$ & & & $0 \mathrm{~m}$ & \\
\hline 09.3 & - & 1 & & 152.3 & $2^{1} P_{1}-b^{3} P^{\prime}{ }_{2}$ & & & & \\
\hline 10.65 & - & $2 \mathrm{~h}$ & & 138.3 & $\left\{\begin{array}{l}2^{3} \mathrm{~F}_{3}-b^{3} \mathrm{P}^{\prime} \\
y^{3} \mathrm{P}_{1}-c^{3} \mathrm{P}_{1}^{\prime}\end{array}\right\}$ & & & & \\
\hline 12.05 & 2 & 4 & $18 \mathrm{~V} \mathrm{E}$ & 123.9 & $a^{1} \mathrm{~S}_{0}-y^{3} \mathrm{P}_{1}$ & & & $\mathrm{~m}$ & \\
\hline 14.45 & - & $10 \mathrm{~h}$ & & $32,099.1$ & $\left\{\begin{array}{l}z^{3} \mathrm{~F}_{2}-\mathrm{c}^{3} \mathrm{D}_{3} ? \\
z^{1} \mathrm{P}_{1}-b^{1} \mathrm{~S}_{0}\end{array}\right.$ & 7 & & & \\
\hline 26.16 & - & $4 \mathrm{~h}$ & & $31,978.9$ & $\left\{\begin{array}{l}y^{3} \mathrm{P}_{2}-c^{3} \mathrm{P}^{\prime}{ }^{3} \mathrm{P}_{1}-c^{3} \mathrm{P}_{0}^{\prime} \\
\mathrm{x}^{2}\end{array}\right.$ & & & & \\
\hline $\begin{array}{l}28.8 \\
30.0 \\
35.17 \\
44.37 \\
60.60\end{array}$ & $\begin{array}{r}3 \\
5 \\
4 \\
- \\
-\end{array}$ & $\begin{array}{l}20 \mathrm{hl} \\
40 \mathrm{hl} \\
5 \\
2 \mathrm{~h} \\
1 \mathrm{~h}\end{array}$ & $\begin{array}{l}5 \mathrm{~V} \mathrm{E} \\
10 \mathrm{VE} \\
25 \mathrm{VEE}\end{array}$ & $\begin{array}{l}951.9 \\
939.7 \\
887.0 \\
793.7 \\
630.4\end{array}$ & $\begin{array}{l}2^{3} \mathrm{~F}_{2}-a^{3} \mathrm{G}_{3} \\
z^{3} \mathrm{~F}_{3}-a^{3} \mathrm{G}_{4} \\
a^{3} \mathrm{D}_{3}-2^{2} \mathrm{~F}_{3} \\
2^{3} \mathrm{~F}_{3}-c^{3} \mathrm{D}_{3} \\
2^{1} \mathrm{P}_{1}-b^{3} \mathrm{P}_{1}^{\prime}\end{array}$ & & & $\begin{array}{l}-1 \mathrm{Nm} \\
-2\end{array}$ & \\
\hline 73.07 & 6 & $100 \mathrm{hl}$ & $10 \mathrm{VE}$ & 506.1 & $2^{3} \mathrm{~F}_{4}-a^{3} \mathrm{G}_{5}$ & $(0.00)-$ & & & \\
\hline 79.42 & 5 & 10 & $40 \mathrm{VE}$ & 443. 2 & $a^{3} \mathrm{D}_{1}-y^{3} \mathrm{P}_{2}$ & $(0,00)-$ & $\left\{\begin{array}{l}(0.00,1.00) \\
0.50,1.50,2.50\end{array}\right.$ & in & \\
\hline $\begin{array}{l}82.42 \\
93.48 \\
95.62\end{array}$ & $\frac{1}{25}$ & $\begin{array}{l}3 \mathrm{hl} \\
2 \mathrm{hl} \\
50\end{array}$ & 100 III E & $\begin{array}{l}413.6 \\
304.8 \\
283.8\end{array}$ & $\begin{array}{l}z^{3} \mathrm{~F}_{3}-c^{3} \mathrm{D}_{2} \\
z^{3} \mathrm{~F}_{2}-a^{1} \mathrm{~F}^{\prime}{ }_{3} \\
a^{3} \mathrm{D}_{1}-y^{3} \mathrm{P}_{1}\end{array}$ & $(0.9 \overline{5}) 1.40$ & $(1.00) 0.50,1.50$ & $2 \mathrm{~h}$ & \\
\hline $\begin{array}{r}3,198.5 \\
3,200.28 \\
03.33 \\
12.40 \\
16.70\end{array}$ & $\begin{array}{r}- \\
25 \\
30 \\
1 \\
50\end{array}$ & $\begin{array}{l}2 \mathrm{~h} \\
50 \\
60 \\
5 \mathrm{hl} \\
100\end{array}$ & $\begin{array}{l}100 \text { III E } \\
100 \text { III E } \\
150 \text { III E }\end{array}$ & $\begin{array}{r}255.6 \\
238.3 \\
208.5 \\
120.4 \\
31,078.8\end{array}$ & $\begin{array}{l}z^{3} \mathrm{P}_{1}-b^{3} \mathrm{D}_{2} \\
a^{3} \mathrm{D}_{2}-y^{3} \mathrm{P}_{2} \\
a^{3} \mathrm{D}_{1}-y^{3} \mathrm{P}_{0} \\
z^{3} \mathrm{D}_{1}^{\prime}-a^{1} \mathrm{P}^{\prime}{ }_{1} \\
a^{3} \mathrm{D}_{2}-y^{3} \mathrm{P}_{1}\end{array}$ & $\begin{array}{l}\text { (w) } 1.26 \mathrm{~B} \\
(0.00) 0.46 \\
(0.00) 0.98\end{array}$ & $\begin{array}{l}(0.50) 1.33 \mathrm{~B} \text { ur } \\
(0.00) 0.50 \\
(0.08) 1.00 \mathrm{~A}^{1} \mathrm{ur}\end{array}$ & $\begin{array}{c}2 \mathrm{Nd} m \\
1 \\
1\end{array}$ & \\
\hline $\begin{array}{l}25.17 \\
31.20 \\
32.00 \\
42.30 \\
80.91\end{array}$ & $\frac{1}{60}$ & $\begin{array}{l}5 \mathrm{hl} \\
2 \mathrm{~h} \\
3 \mathrm{~h} \\
150 \\
? \mathrm{Ag}\end{array}$ & $\begin{array}{l}200 \text { III E } \\
10 \text { V E }\end{array}$ & $\begin{array}{r}30,997.2 \\
939.4 \\
931.7 \\
833.4 \\
470.6\end{array}$ & $\begin{array}{l}z^{3} \mathrm{P}_{2}-b^{3} \mathrm{D}_{3} \\
z^{3} \mathrm{D}_{2}^{\prime}{ }_{2}-b^{3} \mathrm{P}^{\prime}{ }_{2} \\
2^{3} \mathrm{~F}_{4}-c^{3} \mathrm{D}_{3} \\
a^{3} \mathrm{D}_{3}-y^{3} \mathrm{P}_{2} \\
a^{3} \mathrm{P}_{2}^{\prime}{ }_{2}-y^{1} \mathrm{P}_{1}\end{array}$ & $(0.00) 1.18$ & $(0.08) 1.17 \Lambda^{1} u r$ & $1 \mathrm{~b}$ & \\
\hline $\begin{array}{l}82.51 \\
86.71\end{array}$ & - & $\begin{array}{l}2 \\
3 \mathrm{~h}\end{array}$ & & $\begin{array}{l}455.7 \\
416.8\end{array}$ & $\begin{array}{l}z^{3} \mathrm{D}_{{ }_{3}-b^{3} \mathrm{P}_{2}^{\prime}}^{\prime} \\
2^{3} \mathrm{D}_{2}^{\prime}{ }_{2}-b^{3} \mathrm{P}_{1}^{\prime}{ }_{1}\end{array}$ & & & & \\
\hline $\begin{array}{r}3,293.9 \\
3,304.0 \\
08.4\end{array}$ & $\bar{z}$ & $\begin{array}{l}3 \mathrm{~h} \\
2 \mathrm{~h} \\
20 \mathrm{hl}\end{array}$ & & $\begin{array}{l}350.4 \\
257.7 \\
217.4\end{array}$ & $\begin{array}{l}z^{3} D^{\prime}{ }_{1}-c^{3} D_{2} ? \\
z^{3} D^{\prime}{ }_{3}-a^{3} G_{4} \\
z^{3} D^{\prime}{ }_{2}-c^{3} D_{2}\end{array}$ & & & & \\
\hline $\begin{array}{l}12.5 \\
18.6 \\
19.8 \\
27.89 \\
30.9\end{array}$ & $\begin{array}{r}-1 \\
1 \\
50 \\
.2\end{array}$ & $\begin{array}{l}4 \mathrm{hl} \\
4 \mathrm{hl} \\
15 \mathrm{hl} \\
100 \\
20 \mathrm{hl}\end{array}$ & $\begin{array}{l}150 \text { III E } \\
3 \mathrm{VE}\end{array}$ & $\begin{array}{r}180.0 \\
124.6 \\
113.7 \\
040.5 \\
30,013.3\end{array}$ & $\begin{array}{l}z^{3} \mathrm{D}^{\prime}{ }^{\prime}-b^{3} \mathrm{P}^{\prime}{ }_{0} \\
z^{3} \mathrm{D}^{\prime}{ }_{3}-c^{3} \mathrm{D}_{1} \\
z^{3} \mathrm{D}^{\prime}{ }_{3}-c^{3} \mathrm{D}_{3} \\
a^{1} \mathrm{D}_{2}-z^{\mathrm{i}} \mathrm{F}_{3} \\
z^{1} \mathrm{~F}_{3}-b^{1} \mathrm{G}_{4}\end{array}$ & $(0.00) 1.00$ & $(0.00) 1.00$ & 2 & \\
\hline $\begin{array}{c}33.6 \\
35.3 \\
62.00 \\
3,330.1 \\
3,409.9\end{array}$ & $\begin{array}{l}\overline{1} \\
4 \\
1\end{array}$ & $\begin{array}{l}2 \mathrm{~h} \\
4 \mathrm{hl} \\
30 \mathrm{hl} \\
5 \mathrm{hl} \\
4 \mathrm{hl}\end{array}$ & $20 \mathrm{VE}$ & $\begin{array}{r}29,989.0 \\
964.7 \\
735.7 \\
576.5 \\
318.0\end{array}$ & $\begin{array}{l}z^{5} \mathrm{D}^{\prime}{ }_{2}-c^{3} \mathrm{D}_{1} \\
z^{3} \mathrm{D}_{3}^{\prime}-a^{3} \mathrm{G}_{3} \\
b^{1} \mathrm{D}_{2}-y^{1} \mathrm{P}_{1} \\
z^{1} \mathrm{D}^{\prime}{ }_{2}-c^{1} \mathrm{D}_{2} \\
z^{3} \mathrm{D}_{3}^{\prime}-a^{1} \mathrm{~F}^{\prime}{ }_{3}\end{array}$ & $(0.00) 1.10$ & $(0.00) 1.00$ & & \\
\hline $\begin{array}{l}29.4 \\
48.82 \\
57.1 \\
61.0 \\
67.88\end{array}$ & $\begin{array}{l}-\overline{8} \\
1 \\
2 \\
4\end{array}$ & $\begin{array}{l}3 \mathrm{~h} \\
10 \\
4 \mathrm{hl} \\
20 \mathrm{hl} \\
5\end{array}$ & $\begin{array}{l}35 \mathrm{VE} \\
4 \mathrm{~V} ? \\
20 \mathrm{~V} \mathrm{E}\end{array}$ & \begin{tabular}{r|}
$29,151.3$ \\
$28,987.1$ \\
917.7 \\
885.1 \\
827.8
\end{tabular} & $\begin{array}{l}y^{3} \mathrm{P}_{0}-b^{3} \mathrm{~S}_{1} \\
a^{1} \mathrm{D}_{2}-y^{3} \mathrm{P}_{2} \\
y^{3} \mathrm{P}_{2}-b^{3} \mathrm{~S}_{1} \\
2^{1} \mathrm{D}^{\prime}{ }_{2}-b^{3} \mathrm{D}_{2} \\
a^{1} \mathrm{D}_{2}-y^{3} \mathrm{P}_{1}\end{array}$ & (0.72) $1.20 \mathrm{Bw}$ & $(0.75) 1.25 \mathrm{~B}$ ur & $\begin{array}{l}0 \mathrm{~m} \\
-2 ?\end{array}$ & \\
\hline $\begin{array}{c}70.3 \\
3,496.08 \\
3,508.0 \\
44.0 \\
3,549.02\end{array}$ & \begin{tabular}{r|}
40 \\
2 \\
1 \\
50
\end{tabular} & \begin{tabular}{|l}
$5 \mathrm{hl}$ \\
80 \\
$8 \mathrm{hl}$ \\
$3 \mathrm{hl}$ \\
100
\end{tabular} & $\begin{array}{l}150 \text { III E } \\
150 \text { III E }\end{array}$ & $\begin{array}{r}807.7 \\
595.0 \\
498.2 \\
208.7 \\
28,168.8\end{array}$ & $\begin{array}{l}z^{1} \mathrm{D}_{2}^{\prime}-b^{3} \mathrm{D}_{1} \\
a^{1} \mathrm{~S}_{0}-z^{3} \mathrm{D}_{1}^{\prime} \\
2^{3} \mathrm{~F}_{2}-c^{1} \mathrm{D}_{2} \\
2^{1} \mathrm{P}_{1}-c^{1} \mathrm{D}_{2} \\
a^{3} \mathrm{D}_{2}-z^{3} \mathrm{D}_{3}^{\prime}\end{array}$ & $\begin{array}{l}(0.00) 0.62 \\
(0.00) 1.52 \Lambda^{2}\end{array}$ & $\begin{array}{l}(0.00) 0.50 \\
(0.08) 1.50 \mathrm{~A}^{2} \mathrm{ur}\end{array}$ & $\begin{array}{l}0 \mathrm{~m} \\
2\end{array}$ & \\
\hline
\end{tabular}


TABLE 4.-Classified lines of $Y t I I$-Continued

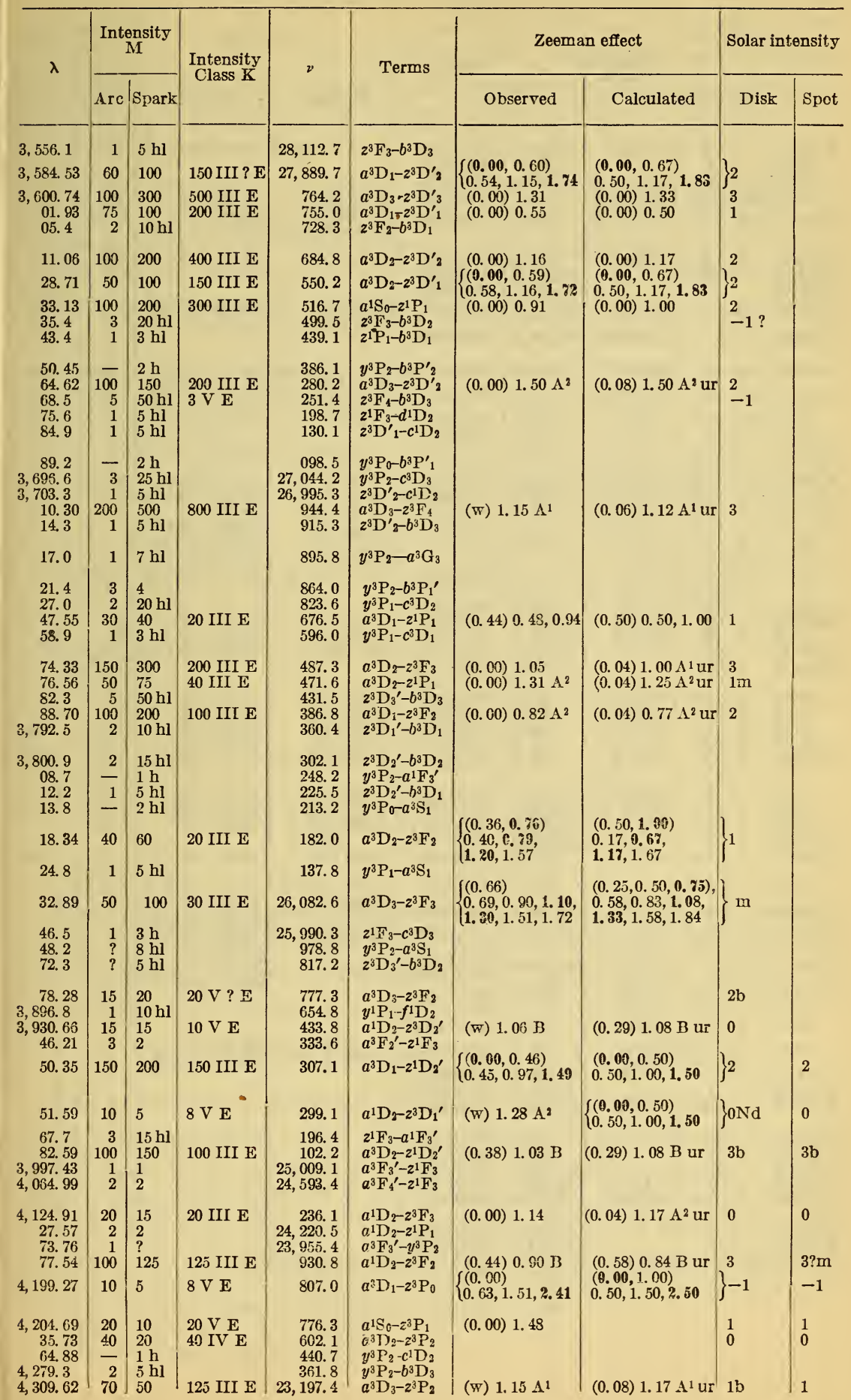


TABLE 4.-Classified lines of $Y t I I$-Continued

\begin{tabular}{|c|c|c|c|c|c|c|c|c|c|}
\hline \multirow{2}{*}{$\lambda$} & \multicolumn{2}{|c|}{$\begin{array}{l}\text { Intensity } \\
\mathbf{M}\end{array}$} & \multirow{2}{*}{$\begin{array}{l}\text { Intensity } \\
\text { Class K }\end{array}$} & \multirow{2}{*}{$\nu$} & \multirow{2}{*}{ Terms } & \multicolumn{2}{|c|}{ Zeeman effect } & \multicolumn{2}{|c|}{ Solar intensity } \\
\hline & Arc & Spark & & & & Observed & Calculated & Disk & Spot \\
\hline $\begin{array}{r}4,358.73 \\
64.01 \\
64.17 \\
74.94\end{array}$ & $\begin{array}{r}40 \\
2 \\
1 \\
200\end{array}$ & $\begin{array}{l}30 \\
? \\
? \\
300\end{array}$ & 300 III E & $\begin{array}{r}22,936.0 \\
908.3 \\
907.4 \\
851.1\end{array}$ & $\begin{array}{l}a^{3} \mathrm{D}_{1}-z^{3} \mathrm{P}_{1} \\
y^{3} \mathrm{P}_{1}-b^{3} \mathrm{D}_{2} \\
y^{3} \mathrm{P}_{0}-b^{3} \mathrm{D}_{1} \\
a^{1} \mathrm{D}_{2}-z^{1} \mathrm{D}_{2}^{\prime}\end{array}$ & $(0.15) 0.95$ & $(0.00) 1.00$ & $2 \mathrm{~m}$ & 2 \\
\hline $4,398.02$ & 75 & 50 & 100 III E & 731.1 & $a^{3} \mathrm{D}_{2}-z^{3} \mathrm{P}_{1}$ & $\left\{\begin{array}{l}(0.00,0.32) \\
0.79,1.10,1.41\end{array}\right.$ & $\begin{array}{l}(0.00,0.33) \\
0.83,1.17,1.50\end{array}$ & \} 1 & 1 \\
\hline $\begin{array}{l}4,422.59 \\
4,465.4 \\
4,607.94\end{array}$ & $\frac{50}{-}$ & $\begin{array}{l}40 \\
10 \mathrm{hl}\end{array}$ & 80 III E & $\begin{array}{r}604.9 \\
22,388.1 \\
21,695.6\end{array}$ & $\begin{array}{l}a^{3} \mathrm{D}_{1}-z^{3} \mathrm{P}_{0} \\
z^{1} \mathrm{~F}_{3}-c^{1} \mathrm{D}_{2} \\
z^{1} \mathrm{~F}_{3}-b^{3} \mathrm{D}_{2} ?\end{array}$ & $(0.00) 0.51$ & $(0.00) 0.50$ & $3 \mathrm{~m}$ & $\mathrm{~m}$ \\
\hline $\begin{array}{l}4,682.32 \\
4,713.26\end{array}$ & $\begin{array}{l}30 \\
-\end{array}$ & $\frac{20}{-}$ & $\begin{array}{l}20 \mathrm{~V} \mathrm{E} \\
\text { (1) H N R }\end{array}$ & $\begin{array}{l}21,351.0 \\
21,210.8\end{array}$ & $\begin{array}{l}a^{1} \mathrm{D}_{2}-z^{3} \mathrm{P}_{2} \\
a^{3} \mathrm{~F}_{2}^{\prime}-z^{3} \mathrm{D}_{3}^{\prime}\end{array}$ & $\left\{\begin{array}{l}(0.89) 0.52, \\
0.95,1.40,1.84\end{array}\right.$ & $\begin{array}{r}(0.50,1.00) 0.50 \\
1.00,1.50,2.00\end{array}$ & $\{1 \mathrm{~b}$ & $1 \mathrm{~b}$ \\
\hline $4,786.58$ & 30 & 20 & 10 IV E & $20,885.9$ & $a^{3} F_{3^{\prime}}-z^{3} D_{3}^{\prime}$ & $(0.65 \mathrm{w}) 1.20 \mathrm{Bw}$ & (0.63) $1.20 \mathrm{~B} \mathrm{ur}$ & $3 \mathrm{~m}$ & $4 \mathrm{~m}$ \\
\hline $4,823.31$ & 40 & 30 & $12 \mathrm{VE}$ & 726.9 & $a^{3} \mathrm{~F}_{2}^{\prime}-z^{3} \mathrm{D}_{2}^{\prime}$ & $\mid\left\{\begin{array}{l}(0.46,0.99) 0.17 \\
0.64,1.13,1.62\end{array} \mid\right.$ & $\begin{array}{l}(0.50,1.00) 0.17 \\
0.67,1.17,1.67\end{array}$ & \}-2 & -3 \\
\hline $\begin{array}{r}54.87 \\
81.44 \\
4,883.69\end{array}$ & $\begin{array}{r}100 \\
2 \\
150\end{array}$ & $\begin{array}{l}150 \\
2 \\
200\end{array}$ & $\begin{array}{l}70 \mathrm{VE} \\
80 \mathrm{VE}\end{array}$ & $\begin{array}{l}592.1 \\
480.0 \\
470.6\end{array}$ & $\begin{array}{l}a^{3} \mathrm{~F}_{2}^{\prime}-z^{3} \mathrm{D}_{1}{ }^{\prime} \\
a^{1} \mathrm{D}_{2}-z^{3} \mathrm{P}_{1} \\
a^{3} \mathrm{~F}_{4}^{\prime}-z^{3} \mathrm{D}_{3}{ }^{\prime}\end{array}$ & $\begin{array}{l}(0.00) 0.72 \\
(0.00) 1.11 \mathrm{~A}^{1}\end{array}$ & $(0.06) 1.12 \mathrm{~A}^{1} \mathrm{ur}$ & 2 & $\begin{array}{l}1 \mathrm{~b} \\
1\end{array}$ \\
\hline $\begin{array}{l}4,900.13 \\
4,982.13 \\
5,087.42\end{array}$ & $\begin{array}{r}125 \\
15 \\
50\end{array}$ & $\begin{array}{l}150 \\
151 \\
1001\end{array}$ & $\begin{array}{l}80 \text { III E } \\
8 \mathrm{~V} \mathrm{E} \\
150 \mathrm{~V} \mathrm{E}\end{array}$ & $\begin{array}{r}401.9 \\
20,066.2 \\
19.650 .9\end{array}$ & $\begin{array}{l}a^{3} \mathrm{~F}_{3}^{\prime}-z^{3} \mathrm{D}_{2}^{\prime} \\
a^{3} \mathrm{~F}_{3}^{\prime}-z^{3} \mathrm{~F}_{4} \\
a^{3} \mathrm{~F}_{4}^{\prime}-z^{3} \mathrm{~F}_{4}\end{array}$ & $\begin{array}{l}(0.00) 0.97 \mathrm{~A}^{1} \\
(\mathrm{w}) 1.56 \mathrm{~A}^{2} \\
(0.00) 1.22\end{array}$ & $\begin{array}{l}(0.04) 1.00 \mathrm{~A}^{1} \mathrm{ur} \\
(0.12) 1.50 \mathrm{~A}^{2} \mathrm{ur} \\
(0.00) 1.25\end{array}$ & $\frac{2}{1}-2$ & $\begin{array}{l}1 \\
-1\end{array}$ \\
\hline $5,119.12$ & 10 & 201 & $15 \mathrm{~V} \mathrm{E}$ & 529.2 & $a^{3} \mathrm{~F}_{2}^{\prime}-z^{3} \mathrm{~F}_{3}$ & $\left\{\begin{array}{l}(0.00,0.40) \\
1.39,1.82\end{array}\right.$ & $\left\{\begin{array}{l}(0.00,0.42,0.83) \\
0.25,0.67,1.08, \\
1.50,1.92\end{array}\right.$ & \}-1 & \\
\hline 23. 21 & 30 & 501 & $40 \mathrm{VE}$ & 513.6 & $a^{3} \mathrm{~F}_{2}^{\prime}-z^{1} \mathrm{P}_{1}$ & $(0.00) 0.52 \mathrm{~A}^{1}$ & $(0.08) 0.50 \Lambda^{1} \mathrm{ur}$ & 0 & -1 \\
\hline $\begin{array}{r}5,196.43 \\
5,200.42 \\
05.73 \\
5,289.82 \\
5,320.78\end{array}$ & $\begin{array}{r}5 \\
40 \\
50 \\
4 \\
2\end{array}$ & $\begin{array}{l}101 \\
60 \\
80 \\
51 \\
41\end{array}$ & $\begin{array}{l}2 \mathrm{VE} \\
60 \mathrm{VE} \\
80 \mathrm{VE} \\
4 \mathrm{VE}\end{array}$ & $\begin{array}{r}238.6 \\
223.9 \\
19,204.3 \\
18,899.0 \\
789.0\end{array}$ & $\begin{array}{l}a^{3} \mathrm{P}_{2^{\prime}-z^{1} \mathrm{~F}_{3}} \\
a^{3} \mathrm{~F}_{2^{\prime}-z^{3} \mathrm{~F}_{2}} \\
a^{3} \mathrm{~F}_{3}^{\prime}-z^{3} \mathrm{~F}_{3} \\
a^{3} \mathrm{~F}_{3}^{\prime}-z^{3} \mathrm{~F}_{2} \\
a^{3} \mathrm{~F}_{4}^{\prime}-z^{3} \mathrm{~F}_{3}\end{array}$ & $\begin{array}{l}(0.00) 0.72 \\
(0.00) 1.07 \\
\text { (w) } 1.73 \mathrm{~A}^{2}\end{array}$ & $\begin{array}{l}(0.00) 0.67 \\
(0.00) 1.08 \\
(0.21) 1.50 \mathrm{~A}^{2} \mathrm{ur}\end{array}$ & $\begin{array}{l}0 \\
0 \\
-2 \\
-2 \mathrm{~N} ?\end{array}$ & $\begin{array}{l}0 \\
0\end{array}$ \\
\hline $\begin{array}{r}5,402.78 \\
73.40 \\
80.75 \\
5,497.42 \\
5,509.91\end{array}$ & $\begin{array}{l}20 \\
10 \\
10 \\
20 \\
30\end{array}$ & $\begin{array}{l}501 \\
201 \\
151 \\
50 \\
301\end{array}$ & $\begin{array}{l}20 \mathrm{VE} \\
10 \mathrm{VE} \\
8 \mathrm{VE} \\
25 \mathrm{VE} \\
40 \mathrm{VE}\end{array}$ & $\begin{array}{l}503.9 \\
265.1 \\
240.6 \\
185.3 \\
144.1\end{array}$ & $\begin{array}{l}b^{1} \mathrm{D}_{2}-z^{1} \mathrm{~F}_{3} \\
a^{3} \mathrm{P}_{1}^{\prime}-y^{3} \mathrm{P}_{2} \\
a^{3} \mathrm{P}_{0}^{\prime}-y^{3} \mathrm{P}_{1} \\
a^{3} \mathrm{P}_{2}^{\prime}-y^{3} \mathrm{P}_{2} \\
a^{3} \mathrm{~F}_{2}^{\prime}-z^{1} \mathrm{D}_{2}^{\prime}\end{array}$ & \begin{tabular}{ll|}
$(0.00)$ & 0.89 \\
$(0.00)$ & 1.48 \\
$(0.00)$ & 1.48 \\
$(0.00)$ & 1.43 \\
$(0.45)$ & $0.76 \mathrm{Bw}$
\end{tabular} & $\begin{array}{ll}(0.00) & 1.00 \\
(0.00) & 1.50 \\
(0.00) & 1.50 \\
(0.00) & 1.50 \\
(0.58) & 0.84 \mathrm{~B} \mathrm{ur}\end{array}$ & $\begin{array}{l}0 \\
-2 \\
-2 \\
-3 \\
0 \mathrm{~m}\end{array}$ & $\underset{\mathrm{m}}{-1}$ \\
\hline $\begin{array}{r}21.70 \\
44.61 \\
5,546.02 \\
5,610.36 \\
5,662.95\end{array}$ & $\begin{array}{r}2 \\
15 \\
8 \\
1 \\
50\end{array}$ & $\begin{array}{l}20 \\
101 \\
101 \\
2 \\
200\end{array}$ & $\begin{array}{l}30 \mathrm{II}+\mathrm{E} \\
25 \mathrm{III}+\mathrm{E} \\
3 \mathrm{~V} \mathrm{E} \\
50 \mathrm{~V} \mathrm{E}\end{array}$ & \begin{tabular}{r|}
105.3 \\
030.5 \\
$18,026.0$ \\
$17,819.2$ \\
653.8
\end{tabular} & $\begin{array}{l}a^{3} \mathrm{P}_{1}^{\prime}-y^{3} \mathrm{P}_{1} \\
a^{3} \mathrm{P}_{1}^{\prime}-y^{3} \mathrm{P}_{0} \\
a^{3} \mathrm{P}_{2^{\prime}-y^{3} \mathrm{P}_{1}} \\
a^{3} \mathrm{~F}_{3}^{\prime}-z^{1} \mathrm{D}_{2}^{\prime} \\
a^{1} \mathrm{G}_{4}-z^{1} \mathrm{~F}_{3}\end{array}$ & $\begin{array}{l}(0.00) 1.46 \\
(0.00) 1.50 \\
(0.00) 1.46 \\
(0.00) 0.98\end{array}$ & $\begin{array}{l}(0.00) 1.50 \\
(0.00) 1.50 \\
(0.00) 1.50 \\
(0.00) 1.00\end{array}$ & $\begin{array}{l}-1 \mathrm{~m} \\
-2 \mathrm{~m} \\
-2 \\
1 \mathrm{~m}\end{array}$ & $\begin{array}{l}-1 \mathrm{~m} \\
2 \mathrm{~m}\end{array}$ \\
\hline $5,728.91$ & 10 & 101 & & 450.5 & $b^{1} \mathrm{D}_{2}-y^{3} \mathrm{P}_{2}$ & $(0.80) 1.27 \mathrm{Bw}$ & $(0.75) 1.25 \mathrm{~B}$ ur & $-1 \mathrm{dm}$ & \\
\hline $5,781.69$ & 5 & 51 & $2 \mathrm{VE}$ & $17,291.2$ & $b^{1} \mathrm{D}_{2-y^{3}} \mathrm{P}_{1}$ & $\left\{\begin{array}{l}(0.00,0.41) \\
0.73 \mathrm{~A}^{1}\end{array}\right.$ & $\begin{array}{l}(0.00,0.50) \\
0.50,1.00,1.50\end{array}$ & & \\
\hline $6,613.74$ & 25 & 20 & $15 \mathrm{~V} \mathrm{E}$ & $15,115.9$ & $a^{3} \mathrm{P}_{2}^{\prime}-z^{3} \mathrm{D}_{3}^{\prime}$ & $(0.00) 1.24 \mathrm{~A}^{1}$ & $(0.08) 1.17 \mathrm{~A}^{1} \mathrm{ur}$ & & \\
\hline $6,795.41$ & 20 & 30 & $15 \mathrm{~V} \mathrm{E}$ & $14,711.8$ & $\left\{\begin{array}{l}a^{3} \mathrm{P}_{1}^{\prime}-z^{3} \mathrm{D}_{2}^{\prime} \\
a^{3} \mathrm{P}_{0}^{\prime}-z^{3} \mathrm{D}_{1}^{\prime}\end{array}\right.$ & \}$(-) 0.83$ & $\left\{\begin{array}{l}(0.00,0.33) \\
0.83,1.17,1.50\end{array}\right.$ & $-2 \mathrm{~d} ?$ & \\
\hline $6,832.49$ & 3 & 4 & $1 \mathrm{VE}$ & 631.9 & $a^{3} \mathrm{P}_{2}^{\prime}-z^{3} \mathrm{D}_{2}^{\prime}$ & & & $-3 ?$ & \\
\hline 58.25 & 3 & 5 & $1 \mathrm{VE}$ & 577.0 & $a^{3} \mathrm{P}_{1}^{\prime}-z^{3} \mathrm{D}_{1}^{\prime}$ & & & & \\
\hline $6,896.00$ & 5 & 10 & $3 \mathrm{VE}$ & 497.2 & $a^{3} \mathrm{P}_{2}^{\prime}-z^{3} \mathrm{D}_{1}^{\prime}$ & (-) 2.21 & $\left\{\begin{array}{l}(0.00,1.00) \\
0.50,1.50,2.50\end{array}\right.$ & & \\
\hline $\begin{array}{l}6,951.68 \\
7,193.74 \\
7,264.19\end{array}$ & $\begin{array}{l}1 \\
1 \\
7\end{array}$ & $\begin{array}{l}3 \\
1 \\
10\end{array}$ & $\begin{array}{l}1 \mathrm{~V} \mathrm{E} \\
8 \mathrm{IV} \mathrm{E}\end{array}$ & $\begin{array}{r}14,381.1 \\
13,897.2 \\
762.5\end{array}$ & $\begin{array}{l}b^{1} \mathrm{D}_{2}-z^{3} \mathrm{D}_{3}^{\prime} \\
b^{1} \mathrm{D}_{2}-z^{3} \mathrm{D}_{2}^{\prime} \\
b^{1} \mathrm{D}_{2}-z^{3} \mathrm{D}_{1}^{\prime}\end{array}$ & & {$\left[0.00,1.00, z_{0} 00\right.$} & & \\
\hline $\begin{array}{l}7,332.97 \\
7,388.46 \\
7,406.23 \\
7,450.32 \\
7,881.90\end{array}$ & $\begin{array}{r}1 \\
1 \\
1 \\
5 \\
20\end{array}$ & $\begin{array}{l}2 \\
1 \\
2 \\
5 \\
10\end{array}$ & $\begin{array}{l}5 \text { III? E } \\
10 \mathrm{~V} E\end{array}$ & $\begin{array}{r}633.3 \\
530.9 \\
498.4 \\
13,418.6 \\
12,683.8\end{array}$ & $\begin{array}{l}a^{3} \mathrm{P}_{0^{\prime}-z^{1} \mathrm{P}_{1}} \\
a^{1} \mathrm{G}_{4}-z^{3} \mathrm{D}_{3^{\prime}} \\
a^{3} \mathrm{P}_{1}^{\prime}-z^{1} \mathrm{P}_{1} \\
a^{3} \mathrm{P}_{2^{\prime}-z^{1} \mathrm{P}_{1}} \\
b^{1} \mathrm{D}_{2}-z^{1} \mathrm{P}_{1}\end{array}$ & & & & \\
\hline $\begin{array}{l}8,066.20 \\
8,835.85\end{array}$ & $\begin{array}{l}3 \\
2\end{array}$ & $?$ & & $\begin{array}{l}12,394.0 \\
11,314.4\end{array}$ & $\begin{array}{l}b^{1} \mathrm{D}_{2}-z^{3} \mathrm{~F}_{2} \\
b^{1} \mathrm{D}_{2}-z^{1} \mathrm{D}_{2}^{\prime}\end{array}$ & & & & \\
\hline
\end{tabular}


The term notation in Table 4 is in common use except for a minor variation in the term coefficients. The even terms have been denoted by the letters $a, b, c, \ldots \ldots, a$ being at the lowest level; the odd terms by $z, y, x \ldots \ldots, z$ being the lowest. This is the only departure from previous custom; it has been adopted on account of the danger of confusion in writing or printing between such combinations as $a^{4} \mathrm{~F}^{\prime}-b^{4} \mathrm{~F}$ and $a^{4} \mathrm{~F}-b^{4} \mathrm{~F}^{\prime}$.

The symbols accompanying intensities and Zeeman effects are those generally understood; their meanings are completely defined in the paper describing the observations on yttrium spectra ${ }^{16}$ except for $u r$, which appears with some of the theoretical Zeeman effects in the last column. This symbol indicates that the magnetic components are unresolved, and the theoretical separations of such fused groups of components are assumed to be increased or decreased by one-fourth the distance from the strongest to the weakest in Back's ${ }^{17} \mathrm{~A}^{1}$ and $\mathrm{A}^{2}$ types, respectively. This rule was found by Russell ${ }^{18}$ to fit the measurements of unresolved Zeeman effects in titanium spectra; it also holds remarkably well for the yttrium spectra presented in Tables 4 and 6 . Some of the resolved patterns give evidence that there are slight deviations from the Landé $g$ values, but a detailed discussion of these should be based on measurements of higher precision. Very few enhanced lines remain unclassified. Those with intensity greater than 2 in the spark are as follows:

\begin{tabular}{|c|c|c|c|}
\hline \multirow{2}{*}{$\lambda$} & \multicolumn{2}{|c|}{ Intensity } & \multirow{2}{*}{$\nu$} \\
\hline & Arc & Spark & \\
\hline $\begin{array}{l}3,407.7 \\
4,734.52\end{array}$ & $=$ & $\begin{array}{l}3 \mathrm{~h} \\
5 \mathrm{~h}\end{array}$ & $\begin{array}{l}29,336.9 \\
21,115.6\end{array}$ \\
\hline $4,829.36$ & & $10 \mathrm{~h}$ & $20,700.9$ \\
\hline
\end{tabular}

The last two may possibly represent $6 \mathrm{P}-6 \mathrm{D}$ of Yt III, but unfortunately this can not be established at present. King and Carter (loc. cit.) published a line at $3,847.87$ (15 IV? E) which is probably an error, since this line has never been recorded by other observers.

\section{THE SPECTRUM OF NEUTRAL YTTRIUM (Yt I)}

The normal state of the neutral atom is represented by a doublet-D term with separation of 530 wave numbers. Transitions between this and higher terms account for most of the lines of Classes I and II which King and Carter (loc. cit.) have found to be strong in the furnace at low temperatures. No absorption data for these lines exist except in the solar spectrum, where many of them appear strengthened

\footnotetext{
16 Meggers, B. S. Jour. Research, 1 (RP12), p. 319; September, 1928.

${ }_{17}$ Back, Zeitschr. f. Phys., 15, p. 212; 1923.

18 Russell, Astrophys. J., 66, p. 308; 1927.
} 
in the sun spots. A low quartet $\mathrm{F}^{\prime}$ term combining with a triad ${ }^{4} \mathrm{D}^{\prime},{ }^{4} \mathrm{~F},{ }^{4} \mathrm{G}^{\prime}$ accounts for a considerable number of strong lines of Classes II and III. The companion ${ }^{4} \mathrm{P}^{\prime}$ term gives several good multiplets. The remaining terms of the low-energy set which have been identified are ${ }^{2} \mathrm{D},{ }^{2} \mathrm{~F}^{\prime}$, and ${ }^{2} \mathrm{G}$. A ${ }^{2} \mathrm{P}$ term belonging to the middle set lies lower than any of these and a triad ${ }^{4} \mathrm{P},{ }^{4} \mathrm{D}^{\prime},{ }^{4} \mathrm{~F}$ somewhat higher. These combine with many high terms of the third set and greatly extend the list of classified lines. They also combine with the fundamental ${ }^{2} \mathrm{D}$ term to give important lines in the red and infra-red. The observations of Zeeman effects, which revealed intersystem combinations, were helpful in unraveling these tangles.

Table 5, which is arranged similarly to Table 3 , gives the terms which have been identified in the arc spectrum of yttrium. A few isolated levels are denoted by $1,2,3, \ldots$; these are probably portions of terms of which the remainder could not be identified. The subscripts denote the inner quantum number; for ease of printing and reading whole numbers a half unit larger than the true $j$ values for levels of even multiplicities are used.

TABLE 5.-Relative terms in the Yt I spectrum

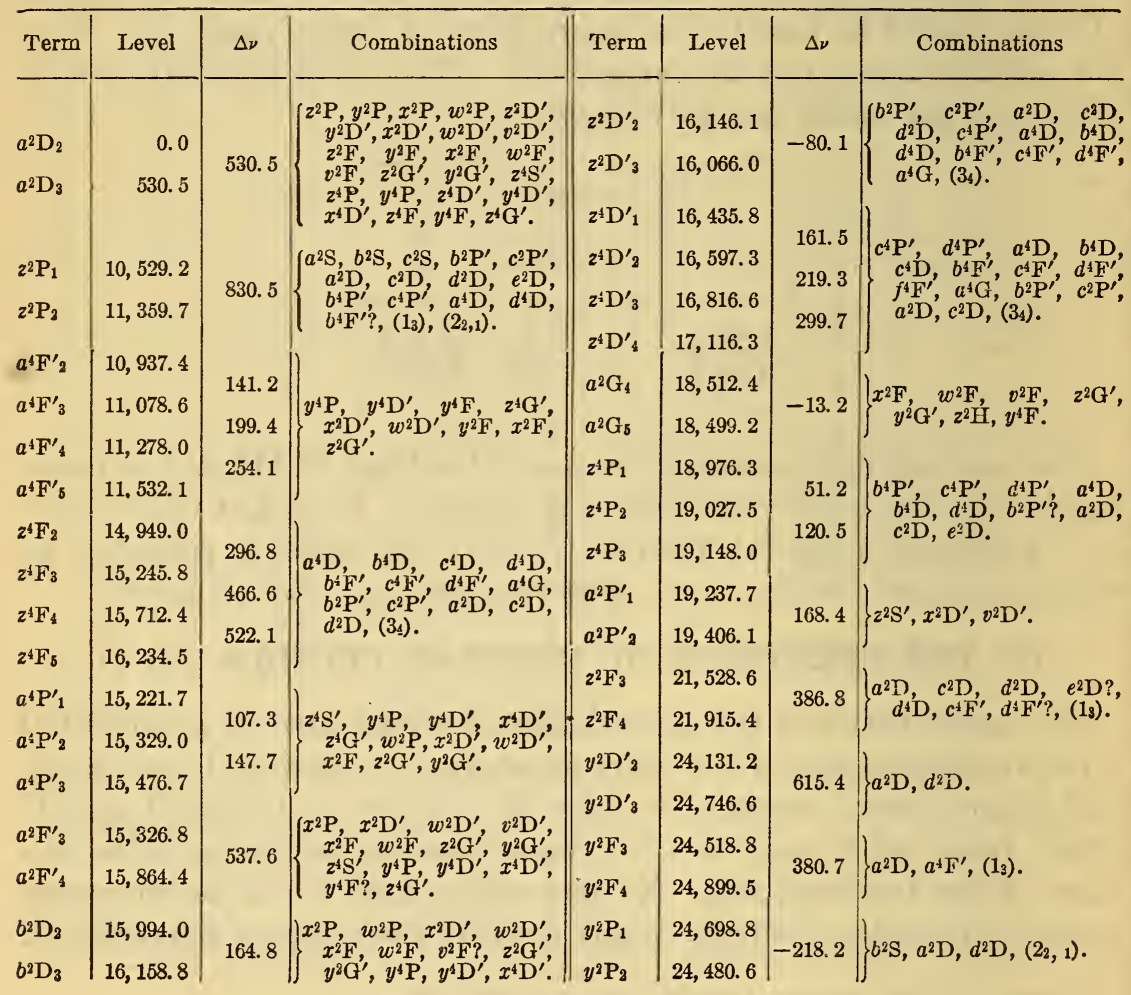


TABLE 5.-Relative terms in the $Y i I$ spectrum-Continued

\begin{tabular}{|c|c|c|c|c|c|c|c|}
\hline Term & Level & $\Delta \nu$ & Combinations & Term & Level & $\Delta \nu$ & Combinations \\
\hline$x^{2} \mathrm{P}_{1}$ & $27,824.5$ & & & $x^{4} \mathrm{D}_{1}^{\prime}$ & $35,816.7$ & & \\
\hline$x^{2} \mathrm{P}_{2}$ & $28,139.6$ & 0. 1 & & $x^{4} \mathrm{D}_{2}^{\prime}$ & $36,135.7$ & $\begin{array}{r}319.0 \\
-74.7\end{array}$ & $a^{4} \mathrm{P}^{\prime}, e^{4} \mathrm{P}^{\prime}, a^{2} \mathrm{D}, \quad b^{2} \mathrm{D}$ \\
\hline $2^{4} \mathrm{G}_{3}^{\prime}$ & $28,694.0$ & 294.9 & & $x^{1} \mathrm{D}^{\prime}{ }_{3}$ & $36,061.0$ & & \\
\hline$z^{4} G_{i}^{\prime}$ & $28,988.9$ & 375.3 & $a^{4} \mathrm{P}^{\prime}, \quad a^{4} \mathrm{~F}^{\prime}, \quad d^{4} \mathrm{~F}^{\prime}, \quad e^{4} \mathrm{~F}^{\prime}$, & $x^{4} \mathrm{D}_{4}^{\prime}$ & $36,361.3$ & & \\
\hline $2^{2} G_{5}^{\prime}$ & $29,364.2$ & 456.2 & . $a^{2} \mathrm{D}, a^{2} \mathrm{~F}^{\prime}$ & $d^{2} \mathrm{D}_{2}$ & $36,420.5$ & 10.5 & $z^{2} \mathrm{P}, \quad y^{2} \mathrm{P}, \quad z^{2} \mathrm{D}^{\prime}, y^{2} \mathrm{D}^{\prime}$, \\
\hline $2^{4} G_{6}^{\prime}$ & $29,820.4$ & & & $d^{2} \mathrm{D}_{3}$ & $36,431.0$ & & $2^{2} \mathrm{~F}, z^{2} \mathrm{~F}$ \\
\hline$b^{4} \mathrm{~F}_{2}^{\prime}$ & $29,272.0$ & 148.3 & & $w^{2} \mathrm{D}_{2}^{\prime}$ & $36,452.3$ & 166.2 & 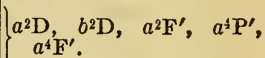 \\
\hline$b^{4} \mathrm{~F}_{3}^{\prime}$ & $29,420.3$ & 193. 3 & $z^{4} \mathrm{D}^{\prime}, z^{4} \mathrm{~F}, z^{2} \mathrm{P} ?, z^{2} \mathrm{D}^{\prime}$ & $w^{2} \mathrm{D}_{3}^{\prime}$ & $36,618.5$ & & 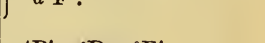 \\
\hline$b^{4} \mathrm{P}_{4}^{\prime}$ & $29,613.6$ & 229.0 & & $2^{4} S_{2}^{\prime}$ & $36,750.8$ & & $a^{4} \mathrm{P}^{\prime}, a^{2} \mathrm{D}, a^{2} \mathrm{~F}^{\prime}$ \\
\hline$b^{4} \mathrm{~F}^{\prime}{ }_{5}$ & $29,842.6$ & & & $y^{\mathbf{4}} \mathrm{P}_{1}$ & $37,039.5$ & 109.0 & 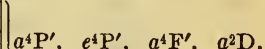 \\
\hline$y^{4} \mathrm{~F}_{2}$ & $31,508.4$ & 171.6 & & $y^{4} \mathrm{P}_{2}$ & $37,148.5$ & 327.5 & $b^{2} \mathrm{D}, a^{2} \mathrm{~F}^{\prime}$ \\
\hline$y^{4} \mathrm{~T}_{3}$ & $31,680.0$ & 229.2 & $\left\{\begin{array}{l}a^{4} \mathrm{~F}^{\prime}, d 4 \mathrm{~F}^{\prime}, e^{4} \mathrm{~F}^{\prime}, \quad a^{2} \mathrm{D}, \\
a^{2} \mathrm{~F}^{\prime} ?, a^{2} \mathrm{G} .\end{array}\right.$ & $y^{2} \mathrm{P}_{3}$ & $37,476.0$ & & \\
\hline$y^{4} \mathrm{~F}_{5}$ & $\begin{array}{l}31,909.2 \\
32,188.1\end{array}$ & 278.9 & & $w^{2} \mathrm{P}_{2}$ & $\begin{array}{l}37,279.3 \\
37,243.7\end{array}$ & -35.6 & $a^{2} \mathrm{D}, b^{2} \mathrm{D}, a^{i} \mathrm{P}^{\prime}$ \\
\hline$a^{2} \mathrm{~S}_{1}$ & $31,671.6$ & & $z^{2} \mathrm{P}$. & $w^{2} F_{3}$ & $37,412.9$ & 206.9 & $a^{2} \mathrm{D}, b^{2} \mathrm{D}, a^{2} \mathrm{~F}^{\prime}, a^{2} \mathrm{G}$ \\
\hline$b^{4} \mathrm{P}_{1}^{\prime}$ & $31,977.5$ & 113.5 & & $w^{2} \mathbf{F}_{4}$ & $37,619.8$ & & (a) \\
\hline$t^{4} \mathrm{P}_{2}^{\prime}$ & $32,091.0$ & 275.3 & $z^{4} \mathrm{P}, z^{2} \mathrm{P}$ & $2^{2} \mathrm{H}_{5}$ & $37,588.2$ & 379.0 & $a^{2} G$ \\
\hline$b^{4} \mathrm{P}^{\prime}{ }_{3}$ & $32,366.3$ & & & ${ }^{2} \mathrm{H}_{4}$ & $37,967.2$ & & \\
\hline$a^{4} \mathrm{D}_{1}$ & $33,148.3$ & 89.5 & & & $38,469.9$ & 73.8 & \\
\hline$a^{4} \mathrm{D}_{2}$ & $33,237.8$ & 173. 7 & $z^{4} \mathrm{P}, z^{4} \mathrm{D}^{\prime}, z^{4} \mathrm{~F}, z^{2} \mathrm{P}, z^{2} \mathrm{D}^{\prime}$ & & $38,543.7$ & 131.7 & $z^{4} \mathrm{P}, z^{4} \mathrm{D}^{\prime}, z^{4} \mathrm{~F}, z^{2} \mathrm{D}^{\prime}$ \\
\hline$a^{4} \mathrm{D}_{3}$ & $33,411.5$ & 341.2 & & $b^{4} \mathrm{D}_{3}$ & $38,675.4$ & 190. 2 & \\
\hline$a^{4} \mathrm{D}_{4}$ & $33,752.7$ & & & $b^{4} \mathrm{D}_{4}$ & $38,865.6$ & & \\
\hline$y^{4} \mathrm{D}_{1}^{\prime}$ & $33,215.4$ & 49.9 & & $y^{2} \mathrm{G}_{4}^{\prime}$ & $38,479.0$ & 117.7 & $\left\{\begin{array}{l}a^{2} \mathrm{D}, b^{2} \mathrm{D}, \quad a^{2} \mathrm{~F}^{\prime}, \quad a^{2} \mathrm{G}, \\
a^{4} \mathrm{P}^{\prime} .\end{array}\right.$ \\
\hline$y^{4} \mathrm{D}_{2}^{\prime}$ & $33,265.3$ & 92.3 & $\left\{\begin{array}{l}a^{4} \mathrm{P}^{\prime}, \quad a^{4} \mathrm{~F}^{\prime}, \quad a^{2} \mathrm{D}, \quad b^{2} \mathrm{D}, \\
a^{2} \mathrm{~F}^{\prime}\end{array}\right.$ & $\begin{array}{l}y^{2} G_{5}^{\prime} \\
a^{4} G_{3}\end{array}$ & $\begin{array}{l}38,596.7 \\
38,635.6\end{array}$ & & \\
\hline$y^{4} \mathrm{D}_{4}^{\prime}$ & $33,357.6$ & 256.9 & & $\begin{array}{l}a^{4} \mathrm{G}_{3} \\
a^{4} \mathrm{G}_{4}\end{array}$ & $\begin{array}{l}38,635.6 \\
38,762.0\end{array}$ & 126.4 & 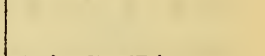 \\
\hline$z^{2} \mathrm{G}^{\prime}{ }_{4}$ & $33,432.3$ & 356.5 & $a^{2} \mathrm{D}, b^{2} \mathrm{D}, a^{2} \mathrm{~F}^{\prime}, \quad a^{2} \mathrm{G}$ & $a^{ \pm} \mathrm{G}_{5}$ & $38,949.3$ & $\begin{array}{l}187.3 \\
273.7\end{array}$ & $z^{4} \mathrm{D}^{\prime} z^{4} \mathrm{~F}, z^{2} \mathrm{D}^{\prime}$ \\
\hline $2^{2} \mathrm{G}_{5}^{\prime}$ & $33,788.8$ & & & $a^{4} \mathrm{G}_{0}$ & $39,223.0$ & & 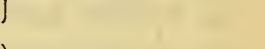 \\
\hline$x^{2} \mathrm{~F}_{3}$ & $33,608.2$ & 421.6 & $\left\{\begin{array}{l}a^{2} \mathrm{D}, \quad b^{2} \mathrm{D}, \quad a^{2} \mathrm{~F}^{\prime}, \quad a^{2} \mathrm{G}, \\
a^{4} \mathrm{P}^{\prime}, a^{4} \Gamma^{\prime} .\end{array}\right.$ & $c^{4} \mathrm{~F}_{2}^{\prime}$ & $39,446.3$ & 118.7 & 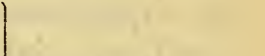 \\
\hline$x^{2} \mathrm{~F}_{4}$ & & & & & & 192.8 & $z^{4} \mathrm{D}^{\prime}, z^{4} \mathrm{~F} \quad z^{2} \mathrm{D}^{\prime}, z^{2} \mathrm{~F}$. \\
\hline$b^{2} \mathrm{P}_{2}^{\prime}$ & $33,013.2$ & 229.1 & $\left\{\begin{array}{l}z^{2} \mathrm{P}, \quad z^{2} \mathrm{D}^{\prime}, \quad z^{6} \mathrm{P} ?, \quad z^{4} \mathrm{D}^{\prime}, \\
z^{4} \mathrm{~F} .\end{array}\right.$ & $c^{4} \mathrm{~F}_{5}^{\prime}$ & $39,963.8$ & 206.0 & \\
\hline$x^{2} \mathrm{D}_{3}^{\prime}$ & $33,906.8$ & 340.9 & $a^{2} \mathrm{P}^{\prime}, a^{2} \mathrm{D}, \quad b^{2} \mathrm{D}, \quad a^{2} \mathrm{~F}^{\prime}$, & $d^{4} \mathrm{P}_{1}^{\prime}$ & & & \\
\hline$x^{2} \mathrm{D}_{3}^{\prime}$ & $34,247.7$ & & & $d^{4} \mathrm{P}_{2}^{\prime}$ & $40,455.1$ & 62.0 & $z^{4} \mathrm{P}, z^{4} \mathrm{D}^{\prime}$ \\
\hline$c^{4} \mathrm{P}_{1}^{\prime}$ & $33,911.5$ & 244.3 & & $d^{4} \mathrm{P}_{3}^{\prime}$ & $40,517.1$ & & \\
\hline$c^{4} \mathrm{P}_{2}^{\prime}$ & $34,155.8$ & 365.4 & $z^{2} \mathrm{P}, z^{4} \mathrm{D}^{\prime}, z^{2} \mathrm{P}, z^{2} \mathrm{D}^{\prime}$ & $v^{2} \mathrm{D}_{2}^{\prime}$ & $40,636.3$ & 36.1 & $a^{2} \mathrm{P}^{\prime}, a^{2} \mathrm{D}, a^{2} \mathrm{~F}^{\prime}$ \\
\hline$c^{4} \mathrm{P}^{\prime}{ }_{3}$ & $34,521.2$ & & & $v^{2} \mathrm{D}_{3}^{\prime}$ & $40,672.4$ & & \\
\hline $\begin{array}{l}c^{2} \mathrm{D}_{2} \\
c^{2} \mathrm{D}_{3}\end{array}$ & $34,231.2$ & 26.2 & $\left\{\begin{array}{l}z^{2} \mathrm{P}, \quad z^{2} \mathrm{D}^{\prime}, \quad z^{2} \mathrm{~F}, \quad z^{4} \mathrm{P}, \\
z^{4} \mathrm{D}^{\prime}, z^{4} \mathrm{~F} .\end{array}\right.$ & $\begin{array}{l}e^{2} \mathrm{D}_{2} \\
e^{2} \mathrm{D}_{3}\end{array}$ & $\begin{array}{l}42,655.8 \\
43,069.8\end{array}$ & 414.0 & $z^{2} \mathrm{P}, z^{2} \mathrm{~F} ?, z^{4} \mathrm{P}$ \\
\hline$z^{2} \mathrm{~S}_{1}^{\prime}$ & $34,438.2$ & & $a^{2} \mathrm{P}^{\prime}$ & $b^{2} \mathrm{~S}_{1}$ & $42,685.5$ & & $z^{2} \mathrm{P}, y^{2} \mathrm{P}$ \\
\hline
\end{tabular}


TABLE 5.-Relative terms in the $Y t I$ spectrum-Continued

\begin{tabular}{|c|c|c|c|c|c|c|c|}
\hline Term & Level & $\Delta \nu$ & Combinations & Term & Level & $\Delta \nu$ & Combinations \\
\hline $\begin{array}{l}v^{2} \mathrm{~F}_{3} \\
v^{2} \mathrm{~F}_{4} \\
d^{4} \mathrm{~F}_{2}^{\prime} \\
d^{4} \mathrm{~F}_{3}^{\prime} \\
d^{4} \mathrm{~F}_{4}^{\prime} \\
d^{4} \mathrm{~F}^{\prime}{ }_{5} \\
c^{2} \mathrm{~S}_{1} \\
e^{4} \mathrm{~F}_{2}^{\prime} \\
e^{4} \mathrm{~F}_{3}^{\prime} \\
e^{4} \mathrm{~F}_{4}^{\prime} \\
e^{4} \mathrm{~F}_{5}^{\prime} \\
c^{4} \mathrm{D}_{1} \\
c^{4} \mathrm{D}_{2} \\
c^{4} \mathrm{D}_{3} \\
c^{4} \mathrm{D}_{4}\end{array}$ & $\begin{array}{c}42,857.9 \\
42,994.8 \\
43,095.7 \\
43,337.5 \\
43,704.4 \\
44,150.5 \\
43,643.9 \\
44,053.0 ? \\
44,365.7 \\
44,759.6 \\
------- \\
---.--- \\
44,655.0 \\
44,922.1\end{array}$ & $\begin{array}{r}241.8 \\
366.9 \\
486.1\end{array}$ & $\begin{array}{l}a^{2} \mathrm{D}, b^{2} \mathrm{D} ?, a^{2} \mathrm{G} . \\
z^{4} \mathrm{D}^{\prime}, z^{4} \mathrm{~F}, y^{4} \mathrm{~F}, z^{4} \mathrm{G}^{\prime}, \\
z^{2}, z^{2} \mathrm{~F} ? . \\
z^{2} \mathrm{P} . \\
y^{4} \mathrm{~F}, z^{4} \mathrm{G}^{\prime} .\end{array}$ & $\begin{array}{l}d^{4} \mathrm{D}_{1} \\
d^{4} \mathrm{D}_{2} \\
d^{4} \mathrm{D}_{3} \\
d^{4} \mathrm{D}_{4} \\
f^{4} \mathrm{~F}_{2}^{\prime} \\
f^{4} \mathrm{~F}_{3}^{\prime} \\
f^{4} \mathrm{~F}_{4}^{\prime} \\
f^{4} \mathrm{~F}^{\prime}{ }_{5} \\
c^{2} \mathrm{P}_{1}^{\prime} \\
c^{2} \mathrm{P}_{2} \\
e^{4} \mathrm{P}_{1}^{\prime} \\
e^{4} \mathrm{P}_{2} \\
e^{4} \mathrm{P}_{3} \\
1_{3} \\
2_{2,1} \\
3_{4}\end{array}$ & $\begin{array}{l}44,660.2 \\
44,748.3 \\
45,008.3 \\
45,204.0 \\
44,742.6 ? \\
45,069.3 \\
45,388.5 \\
45,796.5 \\
45,947.5 \\
45,994.0 \\
-1 . .- \\
-\ldots,-254.0 \\
37,074.1 \\
44,984.5 \\
45,663.5\end{array}$ & $\begin{array}{c}88.1 \\
260.0 \\
195.7 \\
326.7 ? \\
319.2 \\
408.0 \\
46.5\end{array}$ & $\begin{array}{l}z^{4} \mathrm{D}^{\prime} . \\
z^{2} \mathrm{P}, z^{2} \mathrm{D}^{\prime}, z^{4} \mathrm{D}^{\prime}, z^{4} \mathrm{~F} . \\
y^{4} \mathrm{P}, x^{4} \mathrm{D}^{\prime} \\
z^{2} \mathrm{P}, z^{2} \mathrm{~F}, y^{2} \mathrm{~F} . \\
z^{2} \mathrm{P}, y^{2} \mathrm{P} . \\
z^{2} \mathrm{D}^{\prime}, z^{4} \mathrm{D}^{\prime}, z^{4} \mathrm{~F} .\end{array}$ \\
\hline
\end{tabular}

The terms and combinations are represented diagramatically in Figures 2 and 3, where black dots represent doublet terms and circles quartet terms. Full lines represent doublet-doublet combinations and quartet-quartet, and broken lines doublet-quartet combinations. These diagrams are again very similar to those for scandium.

In Table 6 complete details for all classified Yt arc lines are given in the same arrangement and with the same notation as in Table 4. Most of the lines are taken from Meggers's ${ }^{19}$ paper, but it appears that the 220-volt arc employed for these measurements produced a large percentage of ionized atoms and on this account failed to generate the fainter arc lines with appreciable intensity. The list is therefore supplemented by faint lines from Eder's list (labeled E), some from Exner and Haschek's table ( $\mathrm{E}$ and $\mathrm{H}$ ), and a number which were found and measured by one of us (HNR) on the spectrograms made by King and Carter (loc. cit.). All of these are in good agreement with the combinations of well-established terms and may, therefore, be regarded as true arc lines of yttrium. The line $5556.43 \mathrm{~A}$, classified as $z^{4} \mathrm{~F}_{3}-a^{4} \mathrm{D}_{2}$, is abnormally strong in the furnace spectrum and is given by King and Carter as of Class IA. The arc line agrees both in intensity and position with the assigned place in the multiplet. If the furnace line is due to yttrium, it indicates the existence of a

\footnotetext{
19 Meggers, B. S. Jour. Research, 1 (RP 12), p. 319; 1928.
} 
rather low middle level which gives no other levels and for which no theoretical place can be found. It appears probable that it is due to some impurity.

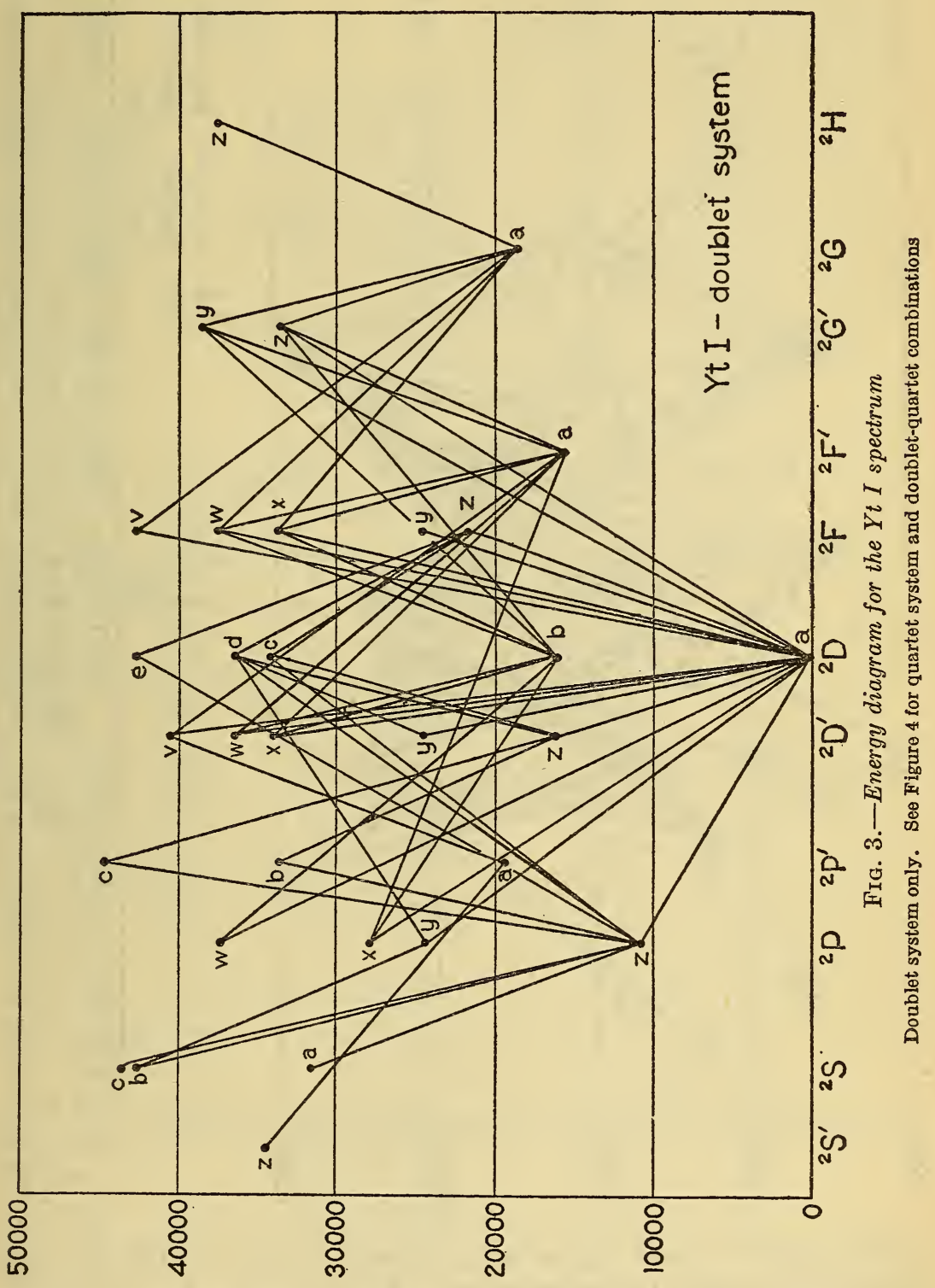

The discordance between observed and calculated Zeeman effects for 3 lines, 4484, 5424, and 5468 A, casts doubt upon the reliability of the observations in these 3 cases. 
The stronger arc lines of yttrium which remain unclassified are listed in Table 7. They are not numerous considering the complexity

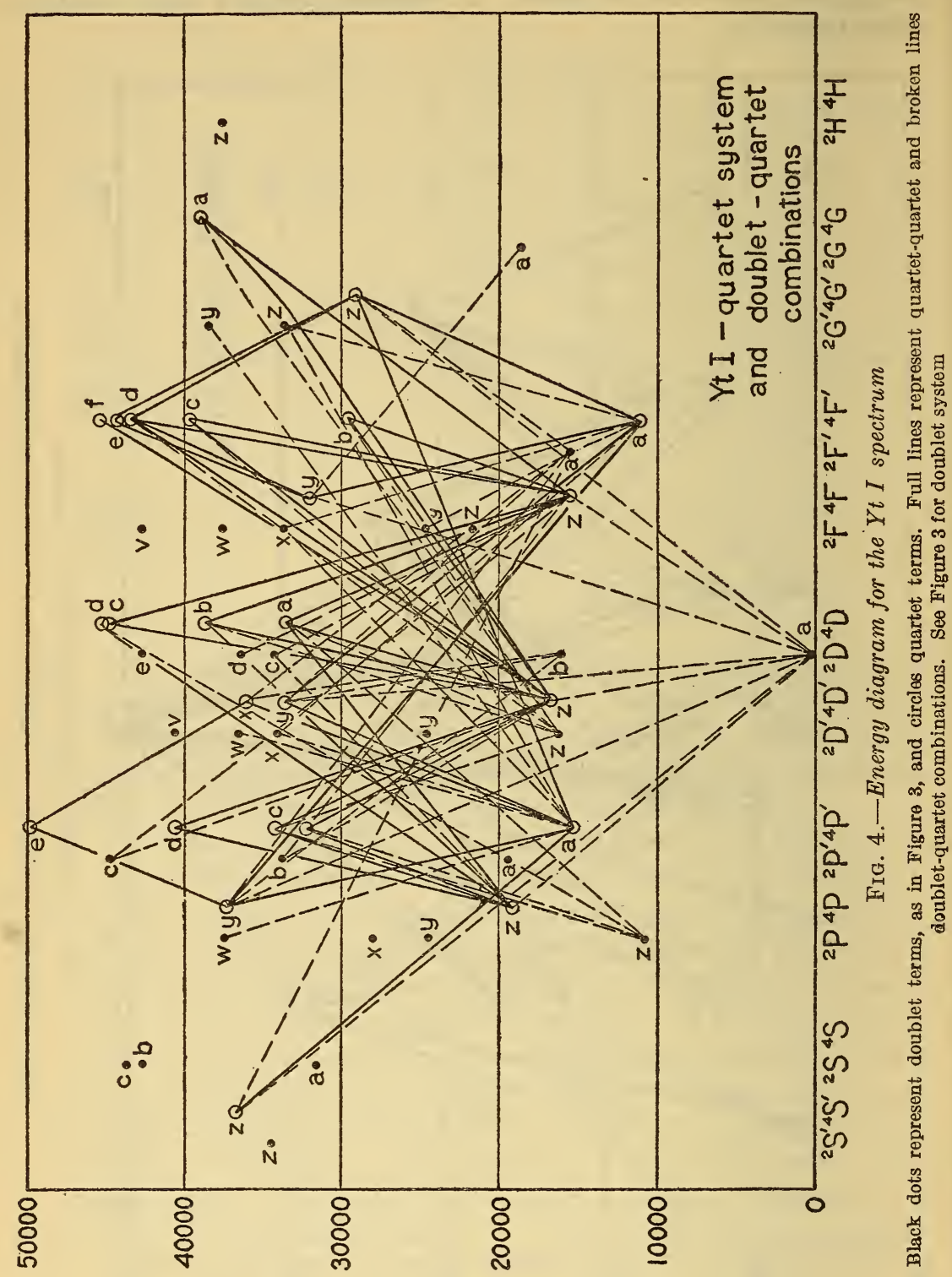

of the spectrum, and almost all of them are weak. Only one line (3587.75 A) reaches the intensity 20 on King's scale or 5 on Meggers's. 
TABLE 6.-Classified lines of $Y t I$

\begin{tabular}{|c|c|c|c|c|c|c|c|c|}
\hline \multirow{2}{*}{$\lambda$} & \multirow{2}{*}{$\begin{array}{c}\text { Intensity } \\
\mathbf{M}\end{array}$} & \multirow{2}{*}{$\begin{array}{c}\text { Intensity } \\
\text { Class K }\end{array}$} & \multirow{2}{*}{$\nu$} & \multirow{2}{*}{ Terms } & \multicolumn{2}{|c|}{ Zeeman effect } & \multicolumn{2}{|c|}{ Solar intensity } \\
\hline & & & & & Observed & Calculated & Disk & Spot \\
\hline $\begin{array}{r}2,332.58 \\
54.20 \\
2,361.81 \\
2,457.93 \\
60.11\end{array}$ & 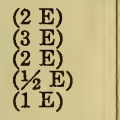 & & $\begin{array}{r}42,857.8 \\
464.3 \\
42,327.5 \\
40,672.3 \\
636.3\end{array}$ & $\begin{array}{l}a^{2} \mathrm{D}_{2}-v^{2} \mathrm{~F}_{3} \\
a^{2} \mathrm{D}_{3}-v^{2} \mathrm{~F}_{4} \\
a^{2} \mathrm{D}_{3}-v^{2} \mathrm{~F}_{3} \\
a^{2} \mathrm{D}_{2}-v^{2} \mathrm{D}_{3}^{\prime} \\
a^{2} \mathrm{D}_{2}-v^{2} \mathrm{D}_{2}^{\prime}\end{array}$ & & & & \\
\hline $\begin{array}{r}2,490.4 \\
2,634.32 \\
72.08 \\
81.65 \\
84.20\end{array}$ & $\begin{array}{l}1 \\
(1 \mathrm{E}) \\
(1 \mathrm{E}) \\
(1 \mathrm{E}) \\
(1 / 2 \mathrm{E})\end{array}$ & & $\begin{array}{r}40,142.1 \\
37,949.2 \\
412.9 \\
279.4 \\
244.0\end{array}$ & $\begin{array}{l}a^{2} \mathrm{D}_{3}-v^{2} \mathrm{D}_{3}^{\prime}{ }_{3} \\
a^{2} \mathrm{D}_{3}-y^{2} \mathrm{G}^{\prime}{ }_{4} \\
a^{2} \mathrm{D}_{2}-v^{2} \mathrm{~F}_{3} \\
a^{2} \mathrm{D}_{2}-w^{2} \mathrm{P}_{1} \\
a^{2} \mathrm{D}_{3}-y^{4} \mathrm{P}_{3}\end{array}$ & & & & \\
\hline $\begin{array}{r}2,695.40 \\
2,705.85 \\
23.00\end{array}$ & 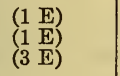 & & $\begin{array}{r}37,089.2 \\
36,946.0 \\
713.3\end{array}$ & $\begin{array}{l}a^{2} \mathrm{D}_{3}-w^{2} \mathrm{~F}_{4} \\
a^{2} \mathrm{D}_{3}-y^{4} \mathrm{P}_{3} \\
a^{2} \mathrm{D}_{3}-w^{2} \mathrm{P}_{2}\end{array}$ & & 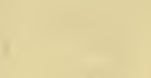 & & \\
\hline 30.06 & (1 E) & & 618.4 & $\left\{\begin{array}{l}a^{2} \mathrm{D}_{2}-w^{2} \mathrm{D}_{3}^{\prime}{ }_{3} \\
a^{2} \mathrm{D}_{3}-y^{4} \mathrm{P}^{2}\end{array}\right.$ & 1 & in & & \\
\hline 42.55 & $(3 \mathrm{E})$ & & 451.7 & $a^{2} \mathrm{D}_{2}-w^{2} \mathrm{D}_{2}^{\prime}$ & & & & \\
\hline $\begin{array}{r}60.10 \\
2,791.20 \\
2,807.66 \\
13.64 \\
18.87\end{array}$ & 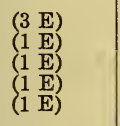 & $\begin{array}{l}1 \text { III A } \\
8 \text { III } \\
3 \text { IV }\end{array}$ & $\begin{array}{r}36,219.9 \\
35,816.3 \\
606.4 \\
531.1 \\
464.8\end{array}$ & $\begin{array}{l}a^{2} \mathrm{D}_{3}-z^{4} \mathrm{~S}^{\prime}{ }_{2} \\
a^{2} \mathrm{D}_{2}-x^{4} \mathrm{D}^{\prime}{ }^{\prime} \\
a^{2} \mathrm{D}_{3}-x^{4} \mathrm{D}^{\prime}{ }_{2} \\
a^{2} \mathrm{D}_{3}-x^{4} \mathrm{D}^{\prime}{ }^{3} \\
z^{2} \mathrm{P}_{1}-c^{2} \mathrm{P}_{2}^{\prime}{ }_{2}\end{array}$ & & & & \\
\hline $\begin{array}{r}22.56 \\
86.49 \\
2,890.40 \\
2,901.48 \\
09.05\end{array}$ & 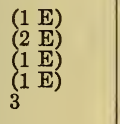 & $\begin{array}{l}10 \mathrm{IV} \\
15 \mathrm{III} \\
3 \mathrm{IV} \\
6 \mathrm{III} \\
20 \mathrm{II}\end{array}$ & $\begin{array}{r}35,418.4 \\
34,634.0 \\
587.2 \\
455.1 \\
247.7\end{array}$ & $\begin{array}{l}z^{2} \mathrm{P}_{1}-c^{2} \mathrm{P}^{\prime}{ }_{1} \\
z^{2} \mathrm{P}_{2}-c^{2} \mathrm{P}^{\prime}{ }_{2} \\
z^{2} \mathrm{P}_{2}-c^{2} \mathrm{P}_{1}^{\prime}{ }_{1} \\
z^{2} \mathrm{P}_{1}-(2,2,1) \\
a^{2} \mathrm{D}_{2}-x^{2} \mathrm{D}^{\prime}{ }_{3}\end{array}$ & & & & \\
\hline $\begin{array}{l}29.00 \\
48.39 \\
64.96 \\
74.59 \\
84.25\end{array}$ & $\begin{array}{l}(1 \mathrm{E}) \\
5 \\
5 \\
6 \\
10\end{array}$ & $\begin{array}{l}30 \mathrm{II} \\
30 \mathrm{II} \\
35 \mathrm{II} \\
50 \mathrm{II}\end{array}$ & $\begin{array}{r}34,131.4 \\
33,906.9 \\
717.5 \\
608.2 \\
499.5\end{array}$ & $\begin{array}{l}z^{2} \mathrm{P}_{1}-d^{4} \mathrm{D}_{1} \\
a^{2} \mathrm{D}_{2}-x^{2} \mathrm{D}^{\prime}{ }_{2} \\
a^{2} \mathrm{D}_{3}-x^{2} \mathrm{D}_{3}^{\prime}{ }_{3} \\
a^{2} \mathrm{D}_{2}-x^{2} \mathrm{~F}_{3} \\
a^{2} \mathrm{D}_{3}-x^{2} \mathrm{~F}_{4}\end{array}$ & 1 & & $-3 ?$ & \\
\hline $\begin{array}{r}95.26 \\
2,996.94 \\
3,005.26 \\
18.95 \\
21.74\end{array}$ & $\begin{array}{l}1 \\
2 \\
1 \\
(2 \mathrm{E}) \\
2\end{array}$ & $\begin{array}{l}10 \text { III } \\
20 \text { III } \\
12 \text { III } \\
6 ? \text { III } \\
15 \text { II }\end{array}$ & $\begin{array}{l}376.4 \\
357.7 \\
265.4 \\
114.5 \\
083.9\end{array}$ & $\begin{array}{l}a^{2} \mathrm{D}_{3}-x^{2} \mathrm{D}_{2}^{\prime} \\
a^{2} \mathrm{D}_{2}-y^{4} \mathrm{D}_{3}^{\prime}{ }_{3} \\
a^{2} \mathrm{D}_{2}-y^{4} \mathrm{D}_{2}^{\prime} \\
z^{2} \mathrm{P}_{1}-c^{2} \mathrm{~S}_{1} \\
a^{2} \mathrm{D}_{3}-y^{4} \mathrm{D}_{4}^{\prime}\end{array}$ & & & $-2 m$ & \\
\hline $\begin{array}{r}22.28 \\
38.46 \\
45.36 \\
3,096.57 \\
3,108.86\end{array}$ & $\begin{array}{l}2 \\
(1 \mathrm{E}) \\
4 \\
(1 \mathrm{E}) \\
(2 \mathrm{E})\end{array}$ & $\begin{array}{l}12 \text { II } \\
4 \text { III A } \\
20 \text { II } \\
3 \text { III } \\
5 \text { III }\end{array}$ & $\begin{array}{r}33,077.9 \\
32,901.9 \\
827.3 \\
284.5 \\
156.8\end{array}$ & $\begin{array}{l}a^{2} \mathrm{D}_{3}-x^{2} \mathrm{~F}_{3} \\
a^{2} \mathrm{D}_{3}-z^{2} \mathrm{G}_{4}^{\prime} \\
a^{2} \mathrm{D}_{3}-y^{4} \mathrm{D}^{\prime}{ }_{3} \\
z^{2} \mathrm{P}_{2}-c^{2} \mathrm{~S}^{\prime}{ }_{2} \\
z^{2} \mathrm{P}_{1}-b^{2} \mathrm{~S}_{1}\end{array}$ & & & $0 \mathrm{~m}$ & \\
\hline $\begin{array}{l}11.80 \\
52.67\end{array}$ & $\stackrel{1}{(2 \mathrm{E})}$ & $\begin{array}{l}15 \text { III } \\
15 \text { n III }\end{array}$ & $\begin{array}{l}32,126.5 \\
31,710.0\end{array}$ & $\begin{array}{l}z^{2} \mathrm{P}_{1}-e^{2} \mathrm{D}_{2} \\
z^{2} \mathrm{P}_{2}-e^{2} \mathrm{D}_{3}\end{array}$ & & & $2 ? \mathrm{~m}$ & \\
\hline $\begin{array}{l}55.62 \\
72.85 \\
85.93\end{array}$ & $\begin{array}{l}\left(\begin{array}{llll}\mathrm{E} & \& & \mathrm{H}\end{array}\right) \\
(1 \mathrm{E} \& \mathrm{H}) \\
(1 \mathrm{E})\end{array}$ & $\begin{array}{l}3 \text { III A } \\
4 \text { III A } \\
2 \text { III A }\end{array}$ & $\begin{array}{l}680.4 \\
508.3 \\
379.0\end{array}$ & $\begin{array}{l}a^{2} \mathrm{D}_{2}-y^{4} \mathrm{~F}_{3} \\
a^{2} \mathrm{D}_{2}-y^{4} \mathrm{~F}_{2} \\
a^{2} \mathrm{D}_{3}-y^{4} \mathrm{~F}_{4}\end{array}$ & . & $\bullet$ & & \\
\hline $\begin{array}{r}91.34 \\
3,194.37 \\
3,209.35 \\
3,251.29 \\
3,337.84\end{array}$ & $\begin{array}{l}2 \\
(2 \mathrm{E}) \\
(2 \mathrm{E}) \\
(2 \mathrm{E}) \\
1\end{array}$ & $\begin{array}{l}15 \text { III } \\
6 \text { III } \\
3 \text { III A }\end{array}$ & $\begin{array}{r}326.0 \\
296.3 \\
31,150.0 \\
30,748.2 \\
29,950.9\end{array}$ & $\begin{array}{l}z^{2} \mathrm{P}_{2}-b^{2} \mathrm{~S}_{1} \\
z^{2} \mathrm{P}_{2}-e^{2} \mathrm{D}_{2} \\
a^{2} \mathrm{D}_{3}-y^{4} \mathrm{~F}_{3} \\
z^{4} \mathrm{~F}_{3}-c^{2} \mathrm{P}^{\prime}{ }_{2} \\
z^{4} \mathrm{~F}_{4}-\left(3_{4}\right)\end{array}$ & & & & \\
\hline $\begin{array}{l}40.37 \\
54.57 \\
54.81 \\
58.94 \\
64.78\end{array}$ & $\begin{array}{l}\left(\begin{array}{ll}3 & \mathrm{E}\end{array}\right) \\
(2 \mathrm{E}) \\
(1 \mathrm{E} \& \mathrm{H}) \\
(2 \mathrm{E}) \\
1\end{array}$ & $\left\{\begin{array}{l}7 \text { III } \\
10 \text { d? III } \\
5 \text { III } \\
7 \text { III }\end{array}\right.$ & $\left\{\begin{array}{l}928.2 \\
801.5 \\
799.4 \\
762.8 \\
711.1\end{array}\right.$ & $\begin{array}{l}z^{2} \mathrm{D}^{\prime}{ }_{3}-c^{2} \mathrm{P}^{\prime}{ }_{2}{ }^{2} \mathrm{D}^{2} \mathrm{D}_{2}-c^{2} \mathrm{P}^{\prime}{ }_{1} \\
z^{4} \mathrm{~F}_{2}-d^{4} \mathrm{D}_{2} \\
z^{4} \mathrm{~F}_{3}-d^{4} \mathrm{D}_{3} \\
z^{4} \mathrm{~F}_{2}-d^{4} \mathrm{D}_{1}\end{array}$ & 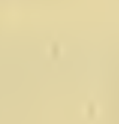 & & & \\
\hline $\begin{array}{r}77.72 \\
88.59 \\
89.81 \\
3,397.04 \\
3,408.17\end{array}$ & $\begin{array}{l}(2 \mathrm{E}) \\
1 \\
(2 \mathrm{E} \& \mathrm{H}) \\
2 \\
(1 \mathrm{E} \& \mathrm{H})\end{array}$ & $\begin{array}{l}6 \text { III } \\
15 \text { III } \\
5 \text { IV? } \\
15 \text { III }\end{array}$ & $\begin{array}{l}597.3 \\
502.3 \\
491.7 \\
429.0 \\
350.1\end{array}$ & $\begin{array}{l}z^{2} \mathrm{D}^{\prime}{ }_{3}-\left(3_{4}\right) \\
z^{4} \mathrm{~F}_{3}-d^{4} \mathrm{D}_{2} \\
z^{4} \mathrm{~F}_{4}-d^{4} \mathrm{D}_{4} \\
z^{4} \mathrm{~F}_{5}^{-}-\left(3_{4}\right) \\
z^{4} \mathrm{D}_{2}^{\prime}-c^{2} \mathrm{P}_{1}^{\prime}\end{array}$ & i & & & \\
\hline $\begin{array}{r}12.47 \\
31.01 \\
50.95 \\
54.17 \\
3,484.06\end{array}$ & $\begin{array}{l}2 \\
(2 \mathrm{E} \& \mathrm{H}) \\
2 \\
(2 \mathrm{E} \& \mathrm{H})\end{array}$ & $\begin{array}{l}12 \mathrm{III} \\
6 \mathrm{~V} \\
12 \mathrm{III} \\
10 \mathrm{III}\end{array}$ & $\begin{array}{r}295.9 \\
29,137.6 \\
28,969.2 \\
942.2 \\
28,694.0\end{array}$ & $\begin{array}{l}z^{4} \mathrm{~F}_{4}-d^{4} \mathrm{D}_{3} \\
z^{2} \mathrm{D}^{\prime} 3^{3}-d^{4} \mathrm{D}_{4} \\
z^{4} \mathrm{~F}_{5}-d^{4} \mathrm{D}_{4} \\
z^{4} \mathrm{~F}_{4}-c^{4} \mathrm{D}_{3} \\
a^{2} \mathrm{D}_{2}-z^{4} \mathrm{G}^{\prime}{ }_{3}\end{array}$ & & & & \\
\hline
\end{tabular}


Table 6.-Classified lines of $Y t I-C o n t i n u e d$

\begin{tabular}{|c|c|c|c|c|c|c|c|c|}
\hline \multirow{2}{*}{$\lambda$} & \multirow{2}{*}{$\underset{M}{\text { Intensity }}$} & \multirow{2}{*}{$\begin{array}{l}\text { Intensity } \\
\text { Class K }\end{array}$} & \multirow{2}{*}{$\nu$} & \multirow{2}{*}{ Terms } & \multicolumn{2}{|c|}{ Zeeman effect } & \multicolumn{2}{|c|}{ Solar intensity } \\
\hline & & & & & Observed & Calculated & Disk & Spot \\
\hline $\begin{array}{r}3,484.89 \\
85.73 \\
3,498.94 \\
3,501.95 \\
10.54\end{array}$ & $\begin{array}{l}2 \\
4 \\
2 \\
2 \\
1\end{array}$ & $\begin{array}{l}40 \text { III } \\
10 \mathrm{n} \mathrm{III} \\
2 \mathrm{IV} \\
8 \mathrm{III}\end{array}$ & $\begin{array}{r}28,687.1 \\
680.2 \\
571.9 \\
547.4 \\
477.5\end{array}$ & 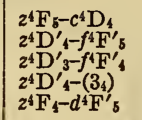 & $(0.00) 1.15$ & $(0.12) 1.17 \mathrm{~A}^{1} \mathrm{ur}$ & $-2 \mathrm{~N} ?$ & \\
\hline 11.19 & 2 & $15 \mathrm{III}$ & 472.2 & $2^{4} D_{2}^{\prime}-f^{4} F_{3}^{\prime}$ & & & & \\
\hline 12.89 & 3 & $20 \mathrm{III}$ & 458.5 & $\left\{\begin{array}{l}z^{4} \overline{\mathrm{F}}_{3}-d^{4} \mathrm{~F}_{4}^{\prime} \\
a^{2} \mathrm{D}_{3}-z^{4} \mathrm{G}_{4}^{\prime}\end{array}\right.$ & $(-) 1.50 \mathrm{~A}^{2}$ & $(0.20) 1.50 \mathrm{~A}^{2} \mathrm{ur}$ & & \\
\hline $\begin{array}{l}21.54 \\
31.71 \\
36.03\end{array}$ & $\begin{array}{l}1 \\
3 \\
1\end{array}$ & $6 \mathrm{III}$ & $\begin{array}{l}388.6 \\
306.8 \\
272.2\end{array}$ & $\begin{array}{c}z^{4} \mathrm{~F}_{2}-d^{4} \mathrm{~F}^{\prime}{ }^{3} \\
2^{4} \mathrm{D}^{\prime}{ }^{1}-f^{4} \mathrm{~F}_{2} ? \\
z^{4} \mathrm{D}^{\prime}{ }_{4}-f^{4} \mathrm{~F}_{4}^{\prime}\end{array}$ & & & 1 & \\
\hline $\begin{array}{l}38.52 \\
51.80 \\
52.70\end{array}$ & $\begin{array}{l}2 \\
2 \\
10\end{array}$ & $\begin{array}{l}8 \mathrm{III} ? \\
40 \mathrm{I}\end{array}$ & $\begin{array}{l}252.4 \\
146.7 \\
139.6\end{array}$ & $\begin{array}{l}z^{4} \mathrm{D}^{\prime}{ }_{3}-f^{4} \mathrm{~F}^{\prime}{ }^{3} \\
z^{4} \mathrm{~F}_{2}-d^{4} \mathrm{~F}^{\prime}{ }^{2} \\
a^{2} \mathrm{D}_{2}-x^{2} \mathrm{P}_{2}\end{array}$ & $\begin{array}{r}(0.80) 0.45 \\
1.00,1.54\end{array}$ & $\begin{array}{l}(0.27,0.80) \\
0.53,1.06,1.60\end{array}$ & & \\
\hline $\begin{array}{l}58.76 \\
71.43\end{array}$ & $\begin{array}{l}2 \\
4\end{array}$ & $\begin{array}{l}5 \mathrm{II} \\
10 \mathrm{II}\end{array}$ & $\begin{array}{l}28,091.7 \\
27,992.0\end{array}$ & $\begin{array}{l}z^{4} F_{3}-d^{4} F^{\prime}{ }^{3} \\
z^{4} F_{4}-d^{4} F^{\prime}{ }_{4}\end{array}$ & (-) 1.22 & $(0.00) 1.24$ & & \\
\hline $\begin{array}{r}76.05 \\
89.68 \\
91.09 \\
92.92 \\
3,595.27\end{array}$ & $\begin{array}{l}5 \\
2 \\
1 \\
25 \\
1\end{array}$ & $\begin{array}{l}12 \text { III } \\
5 ? \text { III? } \\
200 \mathrm{II}\end{array}$ & $\begin{array}{l}955.8 \\
849.7 \\
838.8 \\
824.6 \\
806.4\end{array}$ & $\begin{array}{l}z^{4} \mathrm{~F}_{5}-d^{4} \mathrm{~F}^{\prime}{ }^{4} \\
z^{4} \mathrm{~F}_{3}-d^{4} \mathrm{~F}^{\prime}{ }_{2} \\
z^{4} \mathrm{D}^{\prime}{ }_{3}-c^{4} D_{3} \\
a^{2} \mathrm{D}_{2}-x^{2} \mathrm{P}_{1} \\
z^{4} \mathrm{D}^{\prime}{ }_{4}-c^{4} \mathrm{D}_{4}\end{array}$ & $\begin{array}{l}(-) 1.30 \\
(0.00) 0.84\end{array}$ & $\begin{array}{l}(0.00) 1.33 \\
(0.07) 0.73,0.87\end{array}$ & $0 \mathrm{~m}$ & \\
\hline $\begin{array}{r}3,618.78 \\
20.95 \\
3,639.28 \\
65.75 \\
3,692.53\end{array}$ & $\begin{array}{l}1 \\
50 \\
1 \\
1 \\
3\end{array}$ & $\begin{array}{l}400 \mathrm{II} \\
8 \mathrm{III}\end{array}$ & $\begin{array}{r}625.8 \\
609.3 \\
470.2 \\
271.8 \\
27,074.0\end{array}$ & $\begin{array}{l}z^{4} \mathrm{~F}^{4}-d^{4} \mathrm{~F}^{\prime}{ }^{3} \\
a^{2} \mathrm{D}_{3}-x^{2} \mathrm{P}_{2} \\
z^{4} \mathrm{~F}_{5}-d^{4} \mathrm{~F}^{\prime} \\
z^{2} \mathrm{D}^{\prime}{ }^{3}-d^{4} \mathrm{~F}^{\prime}{ }^{\prime}{ }^{3} \\
z^{4} \mathrm{D}_{4}^{\prime}{ }_{4}-d^{4} \mathrm{~F}_{5}^{\prime}{ }_{5}\end{array}$ & $\begin{array}{l}(0.00) 1.10 \mathrm{~A}^{1} \\
(-) 1.39\end{array}$ & $\begin{array}{l}(0.10) 1.10 \mathrm{~A}^{1} \mathrm{ur} \\
(0.12) 1.50 \mathrm{~A}^{2} \mathrm{ur}\end{array}$ & -1 & \\
\hline $\begin{array}{r}3,718.12 \\
21.40 \\
38.62 \\
49.89 \\
3,787.16\end{array}$ & $\begin{array}{l}2 \\
3 \\
2 \\
2 \\
(2 \mathrm{E} \& \mathrm{H})\end{array}$ & $\begin{array}{l}5 \mathrm{IV} \\
4 \mathrm{III}\end{array}$ & $\begin{array}{r}26,887.7 \\
864.0 \\
740.3 \\
659.9 \\
26,397.5\end{array}$ & $\begin{array}{l}z^{4} \mathrm{D}^{\prime}{ }_{3}-d^{4} \mathrm{~F}^{\prime}{ }^{4} \\
b^{2} \mathrm{D}_{2}-v^{2} \mathrm{~F}_{3} \\
z^{4} \mathrm{D}^{\prime}{ }_{2}-d^{4} \mathrm{~F}^{\prime}{ }^{3} \\
z^{4} \mathrm{D}^{\prime}{ }_{1}-d^{4} \mathrm{~F}^{\prime}{ }_{2} \\
a^{4} \mathrm{~F}^{\prime}{ }_{3}-y^{4} \mathrm{P}_{3}\end{array}$ & & & & \\
\hline $\begin{array}{r}3,887.76 \\
3,892.41 \\
3,900.27 \\
18.24 \\
3,987.49\end{array}$ & $\begin{array}{l}2 \\
(2 \mathrm{E}) \\
(1 \mathrm{E}) \\
2 \\
2\end{array}$ & $\begin{array}{l}4 \mathrm{III} \\
4 \mathrm{III}\end{array}$ & $\begin{array}{r}25,714.5 \\
683.8 \\
632.0 \\
514.5 \\
25,071.4\end{array}$ & $\begin{array}{l}z^{2} \mathrm{P}_{2}-\left(1_{3}\right) \\
z^{4} \mathrm{P}_{1}-d^{4} \mathrm{D}_{1} \\
z^{4} \mathrm{P}_{2}-d^{4} \mathrm{D}_{1} \\
a^{4} \mathrm{~F}^{\prime}{ }_{2}-w^{2} \mathrm{D}^{\prime}{ }_{2} \\
z^{2} \mathrm{P}_{2}-d^{2} \mathrm{D}_{3}\end{array}$ & $(-) 1.00$ & $(0.10) 1.10 \mathrm{~A}^{1} \mathrm{ur}$ & & \\
\hline $\begin{array}{r}4,029.84 \\
39.83 \\
47.64 \\
77.38 \\
80.93\end{array}$ & $\begin{array}{l}2 \\
20 \\
40 \\
100 \\
2\end{array}$ & $\begin{array}{l}3 \mathrm{IV} \\
60 \mathrm{II} \\
80 \mathrm{II} \\
300 \mathrm{II} \\
5 \mathrm{III}\end{array}$ & $\begin{array}{r}24,807.9 \\
746.6 \\
698.8 \\
518.7 \\
497.3\end{array}$ & $\begin{array}{l}a^{2} \mathrm{~F}_{4}^{\prime}-v^{2} \mathrm{D}_{3}^{\prime} \\
a^{2} \mathrm{D}_{2}-y^{2} \mathrm{D}_{3}^{\prime}{ }_{3} \\
a^{2} \mathrm{D}_{2}-y^{2} \mathrm{P}_{1} \\
a^{2} \mathrm{D}_{2}-y^{2} \mathrm{~F}_{3} \\
z^{4} \mathrm{~F}_{2}-c^{4} \mathrm{~F}_{2}^{\prime}\end{array}$ & $\begin{array}{l}(-0.96 \\
(w) 1.40 \mathrm{~A}^{2} \\
(0.00) 0.84 \\
(0.00) 1.00\end{array}$ & $\begin{array}{l}(0.06) 1.07 \mathrm{~A}^{1} \mathrm{ur} \\
(0.30) 1.50 \mathrm{~A}^{2} \mathrm{ur} \\
(0.07) 0.73,0.87 \\
(0.04) 0.90 \mathrm{~A}^{2} \mathrm{ur}\end{array}$ & $\begin{array}{l}-1 \mathrm{~m} \\
0 \mathrm{~N} \\
1 \mathrm{Ndm}\end{array}$ & $\begin{array}{l}0 \mathrm{~m} \\
1 \\
\mathrm{~lm}\end{array}$ \\
\hline 81. 22 & 3 & $9 \mathrm{II}$ & 495.6 & $a^{2} \mathrm{G}_{5}-v^{2} \mathrm{~F}_{4}$ & & & & \\
\hline $4,083.71$ & 50 & $100 \mathrm{II}$ & 480.6 & $a^{2} \mathrm{D}_{2}-y^{2} \mathrm{P}_{2}$ & $\left\{\begin{array}{l}(0.76) 0.53 \\
1.01,1.51\end{array}\right.$ & $\begin{array}{l}(0.27,0.80) \\
0.53,1.06,1.60\end{array}$ & $\{1 \mathrm{~m}$ & $1 \mathrm{~m}$ \\
\hline $\begin{array}{r}4,102.38 \\
4,106.39 \\
10.80\end{array}$ & $\begin{array}{l}150 \\
3 \\
3\end{array}$ & $\begin{array}{l}350 \mathrm{II} \\
5 \mathrm{III}\end{array}$ & $\begin{array}{l}369.2 \\
345.4 \\
319.3\end{array}$ & $\begin{array}{l}a^{2} \mathrm{D}_{3}-y^{2} \mathrm{~F}_{4} \\
a^{2} \mathrm{G}_{4}-v^{2} \mathrm{~F}_{3} \\
z^{4} \mathrm{~F}_{3}-c^{4} \mathrm{~F}_{3}^{\prime}\end{array}$ & $(0.00) 1.08$ & $(0.06) 1.07 \mathrm{~A}^{1} \mathrm{ur}$ & 0 & 2 \\
\hline $\begin{array}{l}28.31 \\
42.86 \\
57.63 \\
58.12\end{array}$ & $\begin{array}{l}150 \\
100 \\
5 \\
(2 \mathrm{E} \& \mathrm{H})\end{array}$ & $\begin{array}{l}300 \mathrm{II} \\
200 \mathrm{II} \\
6 \mathrm{III}\end{array}$ & $\begin{array}{r}216.2 \\
131.2 \\
045.4 \\
24,042.6\end{array}$ & $\begin{array}{l}a^{2} D_{3}-y^{2} D^{\prime}{ }_{3} \\
a^{2} D_{2}-y^{2} D^{\prime}{ }_{2} \\
2^{4} F_{4}-c^{4} F^{\prime}{ }_{4} \\
2^{4} P_{2}-e^{2} D_{3}\end{array}$ & $\begin{array}{l}(0.22) 1.15 \\
(0.00) 0.82 \\
(-0.51,0.85)\end{array}$ & $\begin{array}{l}(0.00) 1.20] \\
(0.00) 0.80 \\
(0.17 .0 .51 .0 .86)\end{array}$ & $\begin{array}{l}-1 \\
-1\end{array}$ & $\begin{array}{l}1 \\
0\end{array}$ \\
\hline 67.52 & 50 & $100 \mathrm{II}$ & $23,988.4$ & $a^{2} \mathrm{D}_{3}-y^{2} \mathrm{~F}_{3}$ & $\left\{\begin{array}{l}0.40,0.74,1.08, \\
1.40,1.73\end{array}\right.$ & $\begin{array}{l}0.34,0.69,1.03 \\
1.37,1.72\end{array}$ & 1 & $1 \mathrm{~m}$ \\
\hline $\begin{array}{r}74.14 \\
4,191.28 \\
4,213.01 \\
13.54 \\
17.80\end{array}$ & $\begin{array}{l}30 \\
1 \\
(1 / 2 \mathrm{E}) \\
2 \\
5\end{array}$ & $\begin{array}{l}100 \mathrm{II} \\
6 \mathrm{IV} \\
5 \mathrm{III} ? \\
10 \mathrm{III}\end{array}$ & $\begin{array}{l}950.3 \\
852.4 \\
729.3 \\
726.4 \\
702.4\end{array}$ & $\begin{array}{l}a^{2} \mathrm{D}_{3}-y^{2} \mathrm{P}_{2} \\
z^{4} \mathrm{~F}_{4}-c^{4} \mathrm{~F}^{\prime}{ }_{3} \\
z^{4} \mathrm{~F}_{5}-c^{4} \mathrm{~F}^{\prime}{ }_{5} \\
z^{4} \mathrm{~F}_{2}-b^{4} \mathrm{D}_{3} \\
z^{2} \mathrm{P}_{1}-c^{2} \mathrm{D}_{3}\end{array}$ & $\begin{array}{l}(0.00) 1.10 \mathrm{~A}^{1} \\
(-) 1.06\end{array}$ & $(0.07) 0.73,0.87_{t}$ & - & -1 \\
\hline $\begin{array}{r}20.62 \\
29.20 \\
31.30 \\
32.54 \\
4,235.94\end{array}$ & $\begin{array}{l}10 \\
2 \\
(1 \mathrm{E} \& \mathrm{H}) \\
2 \\
20\end{array}$ & $\begin{array}{l}30 \mathrm{III} \\
2 \mathrm{III} \\
1 \mathrm{III} \\
100 \mathrm{II}\end{array}$ & $\begin{array}{r}686.6 \\
638.5 \\
626.8 \\
619.9 \\
23,600.9\end{array}$ & $\begin{array}{l}z^{4} \mathrm{~F}_{2}-a^{4} \mathrm{G}_{3} \\
z^{4} \mathrm{D}_{3}^{\prime}-d^{4} \mathrm{P}^{\prime}{ }_{2} \\
z^{2} \mathrm{P}_{1}-c^{4} \mathrm{P}_{2}^{\prime} \\
z^{4} \mathrm{~F}_{3}-b^{4} \mathrm{D}_{4} \\
a^{2} \mathrm{D}_{3}-y^{2} \mathrm{D}_{2}\end{array}$ & $\begin{array}{c}\text { (w?) } 1.03 \mathrm{~B} ? \\
=\end{array}$ & $(0.13) 0.70 \mathrm{~A}^{2} \mathrm{ur}$ & m & m \\
\hline
\end{tabular}


TABle 6.-Classified lines of Yt I-Continued

\begin{tabular}{|c|c|c|c|c|c|c|c|c|}
\hline \multirow{2}{*}{$\lambda$} & \multirow{2}{*}{$\underset{\mathbf{M}}{\text { Intensity }}$} & \multirow{2}{*}{$\begin{array}{c}\text { Intensity } \\
\text { Class K }\end{array}$} & \multirow{2}{*}{$\nu$} & \multirow{2}{*}{ Terms } & \multicolumn{2}{|c|}{ Zeeman effect } & \multicolumn{2}{|c|}{ Solar intensity } \\
\hline & & & & & Observed ${ }^{\circ}$ & Calculated & Disk & Spot \\
\hline $\begin{array}{r}4,237.12 \\
49.87 \\
50.36 \\
51.19 \\
54.35\end{array}$ & $\begin{array}{l}\left.\left(\begin{array}{l}1 \\
\mathbf{E}\end{array}\right) \mathrm{H}\right) \\
1 \\
10 \\
1\end{array}$ & $\begin{array}{l}1 \mathrm{III} \\
1 \mathrm{III} \\
40 \mathrm{III}\end{array}$ & $\begin{array}{r}23,594.3 \\
523.5 \\
520.8 \\
516.2 \\
498.8\end{array}$ & $\begin{array}{l}z^{4} \mathrm{~F}_{2}-b^{4} \mathrm{D}_{2} \\
2^{4} \mathrm{~F}_{5}-c^{4} \mathrm{~F}^{\prime}{ }_{4} \\
z^{2} \mathrm{~F}_{2}-b^{4} \mathrm{D}_{1} \\
2^{4} \mathrm{~F}_{3}-a^{4} \mathrm{G}_{4} \\
2^{2} \mathrm{D}^{\prime}{ }_{3}-c^{4} \mathrm{~F}^{\prime}{ }_{3}\end{array}$ & $(0.00) 0.96$ & $(0.04) 0.93 \mathrm{~A}^{1} \mathrm{ur}$ & & \\
\hline $\begin{array}{l}66.89 \\
68.84 \\
72.12 \\
74.18 \\
75.50\end{array}$ & $\begin{array}{l}1 \\
(1 \mathrm{E} \& \mathrm{H}) \\
2 \\
1 \\
1(\mathrm{E} \& \mathrm{H})\end{array}$ & $\begin{array}{l}1 \text { III } \\
2 \text { III } \\
1 \text { III A }\end{array}$ & $\begin{array}{l}429.7 \\
419.0 \\
401.0 \\
389.7 \\
382.5\end{array}$ & $\begin{array}{l}z^{4} \mathrm{~F}_{3}-b^{4} \mathrm{D}_{3} \\
z^{2} \mathrm{D}^{\prime}{ }_{2}-c^{4} \mathrm{~F}^{\prime}{ }_{3} \\
z^{4} \mathrm{D}^{\prime}{ }_{4}-d^{4} \mathrm{P}^{\prime}{ }_{3} \\
2^{4} \mathrm{~F}_{3}-a^{4} \mathrm{G}_{3} \\
2^{2} \mathrm{P}_{1}-c^{4} \mathrm{P}^{\prime}{ }_{1}\end{array}$ & $(0.00) 1.14$ & $(0.17) 1.21 \mathrm{~A}^{1} \mathrm{ur}$ & & \\
\hline $\begin{array}{r}88.20 \\
91.04 \\
4,292.79\end{array}$ & 2 & $2 \underset{\mathrm{III}}{(0 \mathrm{H} \mathrm{R})}$ & $\begin{array}{l}313.3 \\
297.8 \\
288.3\end{array}$ & $\begin{array}{l}z^{2} \mathrm{P}_{1}-b^{2} \mathrm{P}_{2}^{\prime} \\
z^{4} \mathrm{~F}_{3}-b^{4} \mathrm{D}_{2} \\
z^{2} \mathrm{~F}_{4}-d^{4} \mathrm{D}_{4}\end{array}$ & $(-) 0.85$ & $(0.12) 0.90 \mathrm{~A}^{1} \mathrm{ur}$ & & \\
\hline $\begin{array}{r}4,292.79 \\
4,302.30 \\
14.92\end{array}$ & 20 & $\begin{array}{l}50 \mathrm{III} \\
(1 \mathrm{H} \mathrm{N} \mathrm{R})\end{array}$ & $\begin{array}{l}288.3 \\
236.9 \\
168.9\end{array}$ & $\begin{array}{l}z^{4} \mathrm{~F}_{4}-a^{4} \mathrm{G}_{5} \\
a^{4} \mathrm{~F}^{\prime}{ }_{3}-x^{2} \mathrm{D}_{3}^{\prime}\end{array}$ & $(0.52 \mathrm{w}) 1.04 \mathrm{~A}^{2}$ & $(0.08) 1.05 \mathrm{~A}^{1} \mathrm{ur}$ & $2 \mathrm{~m}$ & $2 \mathrm{~m}$ \\
\hline $\begin{array}{l}16.30 \\
17.87 \\
18.21 \\
30.78 \\
37.30\end{array}$ & $\begin{array}{l}2 \\
1 \\
1 \\
5 \\
2\end{array}$ & $\begin{array}{l}3 \text { III } \\
10 \text { III } \\
2 \text { III }\end{array}$ & $\begin{array}{l}161.5 \\
153.1 \\
152.1 \\
084.1 \\
049.4\end{array}$ & $\begin{array}{l}z^{2} \mathrm{P}_{2}-c^{4} \mathrm{P}^{\prime}{ }_{3} \\
z^{4} \mathrm{~F}_{4}-b^{4} \mathrm{D}_{4} \\
a^{2} \mathrm{~F}^{\prime}-y^{2} \mathrm{G}^{\prime}{ }_{4} \\
z^{2} \mathrm{P}_{1}-b^{2} \mathrm{P}^{\prime}{ }_{1} \\
z^{4} \mathrm{~F}_{4}-a^{4} \mathrm{G}_{4}\end{array}$ & $\begin{array}{l}(0.00) 0.73 \\
(-) 1.13 \mathrm{~B}\end{array}$ & $\begin{array}{l}(0.00) 0.67 \\
(0.70) 1.11 \mathrm{~B} \mathrm{ur}\end{array}$ & & \\
\hline $\begin{array}{l}44.64 \\
46.14\end{array}$ & $\begin{array}{l}3 \\
1\end{array}$ & 4 III & $\begin{array}{r}010.4 \\
23,002.5\end{array}$ & $\begin{array}{l}z^{4} \mathrm{D}^{\prime}{ }_{1}-c^{4} \mathrm{~F}^{\prime}{ }^{2} \\
a^{4} \mathrm{P}^{\prime}{ }_{3}-y^{2} \mathrm{G}^{\prime}\end{array}$ & $(-) 0.83 \mathrm{~A}^{2}$ & $(0.20) 0.20,0.60$ & & \\
\hline 48.78 & 25 & $60 \mathrm{III}$ & $22,988.5$ & $z^{4} \mathrm{~F}_{5}-a^{4} \mathrm{G}_{6}$ & $(0.00) 1.10$ & $(0.09) 1.14 \mathrm{~A}^{1} \mathrm{ur}$ & $-1 N ?$ & $\mathrm{~m}$ \\
\hline 52.33 & 2 & 5 III & 969.8 & 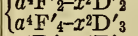 & \}$(-) 1.27$ & $(0.04) 1.25 \mathrm{~A}^{2} \mathrm{ur}$ & & \\
\hline 52.68 & 4 & $4 \mathrm{III}$ & 967.9 & $2^{4} \mathrm{D}_{2}^{\prime}-c^{4} \mathrm{~F}_{3}^{\prime}$ & $(-) 0.96$ & $(0.12) 0.90 \mathrm{~A}^{1} \mathrm{ur}$ & & \\
\hline $\begin{array}{l}53.63 \\
57.73 \\
61.18 \\
66.03\end{array}$ & $\begin{array}{l}2 \\
6 \\
1 \\
5\end{array}$ & $\begin{array}{l}10 \mathrm{III} \\
10 \mathrm{III}\end{array}$ & $\begin{array}{l}962.9 \\
941.3 \\
923.2 \\
897.7\end{array}$ & $\begin{array}{l}z^{4} \mathrm{~F}_{4}-b^{4} \mathrm{D}_{3} \\
z^{4} \mathrm{D}^{\prime}{ }_{3}-c^{4} \mathrm{~F}^{\prime}{ }_{4} \\
z^{4} \mathrm{~F}_{4}-a^{4} \mathrm{G}_{3} \\
z^{2} \mathrm{P}_{2}-c^{2} \mathrm{D}_{3}\end{array}$ & $\begin{array}{l}(-) 0.98 \\
(0.00) 1.18\end{array}$ & $\begin{array}{l}\text { (0.13) } 1.07 \mathrm{~A}^{1} \mathrm{ur} \\
(0.10) 1.10 \mathrm{~A}^{1} \mathrm{ur}\end{array}$ & -2 & \\
\hline 70.97 & 1 & $1 \mathrm{III}$ & 871.8 & $z^{2} \mathrm{P}_{2}-c^{2} \mathrm{D}_{2}$ & $(-) 0.98$ & $\left\{\begin{array}{l}(0.27,0.80) 0.53, \\
1.06,1.60\end{array}\right.$ & & \\
\hline $\begin{array}{l}75.61 \\
79.34 \\
84.80\end{array}$ & $\begin{array}{l}3 \\
3 \\
2\end{array}$ & $\begin{array}{l}8 \text { III } \\
6 \text { III }\end{array}$ & $\begin{array}{l}847.6 \\
828.1 \\
799.7\end{array}$ & $\begin{array}{l}z^{4} \mathrm{D}_{4}^{\prime}{ }_{4}-c^{4} \mathrm{~F}^{\prime}{ }_{5} \\
a^{4} \mathrm{~F}^{\prime}{ }_{3}-x^{2} \mathrm{D}^{\prime}{ }_{2} \\
z^{2} \mathrm{D}^{\prime}{ }_{3}-b^{4} \mathrm{D}_{4}\end{array}$ & $(-) 1.19 \mathrm{~A}^{2}$ & $(0.16) 1.20 \mathrm{~A}^{2} \mathrm{ur}$ & & \\
\hline 85.48 & 2 & $3 \mathrm{III}$ & 796.1 & $z^{2} \mathrm{P}_{2}-c^{4} \mathrm{P}_{2}^{\prime}$ & $(-) 1.36$ & $\left\{\begin{array}{l}(0.20,0.60) 1.13, \\
1.53,1.93\end{array}\right.$ & & \\
\hline 94.01 & 2 & $3 \mathrm{III}$ & 751.9 & $a^{4} \mathrm{~F}_{4}^{\prime}-x^{2} \mathrm{~F}_{4}$ & $(-) 1.17 \mathrm{~B}$ & $(0.12) 1.19 \mathrm{~B} \mathrm{ur}$ & & \\
\hline $\begin{array}{r}94.67 \\
4,397.79 \\
4,401.13 \\
02.38 \\
04.85\end{array}$ & $\begin{array}{l}2 \\
(2 \mathrm{E} \& \mathrm{H}) \\
2 \\
3 \\
3\end{array}$ & 2 III & $\begin{array}{l}748.5 \\
732.3 \\
715.1 \\
708.6 \\
695.9\end{array}$ & $\begin{array}{l}z^{4} \mathrm{D}^{\prime}{ }_{3}-c^{4} \mathrm{~F}^{\prime}{ }_{3}{ }^{2} \mathrm{~F}^{\prime}{ }_{4}-y^{2} \mathrm{G}^{\prime}{ }_{5} \\
z^{4} \mathrm{~F}_{5}-a^{4} \mathrm{G}_{5} \\
z^{2} \mathrm{P}_{1}-a^{4} \mathrm{D}_{2} \\
z^{2} \mathrm{D}^{\prime}{ }_{3}-a^{4} \mathrm{G}_{4}\end{array}$ & & & & \\
\hline $\begin{array}{l}09.71 \\
15.37 \\
17.44 \\
21.74 \\
32.92\end{array}$ & $\begin{array}{l}1 \\
2 \\
2 \\
1 \\
1\end{array}$ & $\begin{array}{l}3 \text { III } \\
2 \text { III } \\
1 \text { IV } \\
1 \text { IV }\end{array}$ & $\begin{array}{l}670.9 \\
641.8 \\
631.2 \\
609.2 \\
552.2\end{array}$ & $\begin{array}{l}a^{4} \mathrm{~F}^{\prime}{ }_{2}-x^{2} \mathrm{~F}_{3} \\
z^{4} \mathrm{D}^{\prime}{ }_{4}-c^{4} \mathrm{~F}^{\prime}{ }_{4} \\
z^{4} \mathrm{~F}_{5}-b^{4} \mathrm{D}_{4} \\
z^{2} \mathrm{D}^{\prime}{ }_{3}-b^{4} \mathrm{D}_{3} \\
z^{2} \mathrm{P}_{2}-c^{4} \mathrm{P}_{1}^{\prime}\end{array}$ & $\begin{array}{l}\text { (w) } 1.23 \mathrm{~B} \mathrm{w} \\
\text { (-) } 1.22\end{array}$ & $\begin{array}{l}(0.52) 1.33 \mathrm{~B}{ }^{1} \mathrm{rr} \\
(0.12) 1.16 \mathrm{~A}^{1} \mathrm{ur}\end{array}$ & & \\
\hline $\begin{array}{l}36.13 \\
37.34 \\
43.66 \\
45.31 \\
46.63\end{array}$ & $\begin{array}{l}1 \\
5 \\
7 \\
3 \\
8\end{array}$ & $\begin{array}{l}10 \mathrm{III} \\
15 \mathrm{III} \\
3 \mathrm{IV} \\
15 \mathrm{III}\end{array}$ & $\begin{array}{l}535.8 \\
529.7 \\
497.7 \\
489.3 \\
482.6\end{array}$ & $\begin{array}{l}a^{4} \mathrm{~F}^{\prime}{ }^{3}-y^{4} \mathrm{D}^{\prime}{ }_{4} \\
a^{4} \mathrm{~F}^{3}{ }^{3}-x^{2} \mathrm{~F}_{3} \\
a^{4} \mathrm{~F}^{\prime}{ }_{5}-x^{2} \mathrm{~F}_{4} \\
z^{2} \mathrm{D}^{\prime}{ }_{2}-a^{4} \mathrm{G}_{3} \\
z^{2} \mathrm{P}_{2}-b^{2} \mathrm{P}_{2}^{\prime}\end{array}$ & 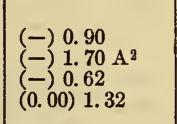 & $\begin{array}{l}(0.35) 0.94 \mathrm{~B} \mathrm{ur} \\
(0.24) 1.66 \mathrm{~A}^{2} \mathrm{ur} \\
(0.16) 0.40 \mathrm{~A}^{1} \mathrm{ur} \\
(0.00) 1.33\end{array}$ & $-2 m$ & \\
\hline $\begin{array}{l}59.01 \\
63.53 \\
75.72 \\
76.96\end{array}$ & $\begin{array}{l}1 \\
1(\mathrm{E} \& \mathrm{H}) \\
10 \\
10\end{array}$ & $\begin{array}{l}20 \text { III } \\
25 \text { III }\end{array}$ & $\begin{array}{l}420.2 \\
397.5 \\
336.5 \\
330.3\end{array}$ & $\begin{array}{l}a^{4} \mathrm{~F}^{\prime}{ }_{2}-y^{4} \mathrm{D}^{\prime}{ }_{3} \\
2^{2} \mathrm{D}^{\prime}{ }_{2}-b^{4} \mathrm{D}_{2} \\
a^{4} \mathrm{~F}^{\prime}{ }_{4}-y^{4} \mathrm{D}^{\prime}{ }_{4} \\
a^{4} \mathrm{~F}^{\prime}{ }_{4}-x^{2} \mathrm{~F}_{3}\end{array}$ & $\begin{array}{l}(0.50) 1.29 \mathrm{~B} \text { w } \\
(-) 1.66 \mathrm{~A}^{2}\end{array}$ & $\begin{array}{l}(0.53) 1.33 \mathrm{~B} \mathrm{ur} \\
(0.38) 1.72 \mathrm{~A}^{2} \mathrm{ur}\end{array}$ & $-2 \mathrm{~N}$ & \\
\hline 77.45 & 10 & $25 \mathrm{III}$ & 327.9 & $a^{4} \mathrm{~F}_{2}^{\prime}{ }_{2}-\mathrm{D}^{4} \mathrm{D}_{2}^{\prime}$ & $\left\{\begin{array}{l}(-) \\
1.54\end{array}\right.$ & $\left\{\begin{array}{l}(0.40,1.20) \\
0.80,1.60\end{array}\right.$ & \}$-1 N$ & -1 \\
\hline $\begin{array}{l}78.99 \\
84.44 \\
87.28 \\
87.47 \\
91.75\end{array}$ & $\begin{array}{l}1 \\
2 \\
8 \\
15 \\
3\end{array}$ & $\begin{array}{l}2 \text { IV } \\
5 \text { II? } \\
20 \text { III } \\
40 \text { III } \\
5 \text { III }\end{array}$ & $\begin{array}{l}320.2 \\
293.1 \\
279.0 \\
278.0 \\
256.8\end{array}$ & $\begin{array}{l}b^{2} \mathrm{D}_{3}-y^{2} \mathrm{G}^{\prime}{ }_{4} \\
a^{2} \mathrm{~F}^{\prime}{ }^{3}-w^{2} \mathrm{~F}_{4} ? \\
a^{4} \mathrm{~F}^{\prime}{ }_{3}-y^{4} \mathrm{D}^{\prime}{ }_{3} \\
a^{4} \mathrm{~F}^{\prime}{ }^{\prime}-y^{4} \mathrm{D}^{\prime}{ }_{1} \\
a^{4} \mathrm{~F}^{\prime}{ }_{5}-z^{2} \mathrm{G}_{5}{ }_{5}\end{array}$ & $\begin{array}{l}(-) 1.06 \\
(\mathrm{w}) 1.20 \mathrm{~B} \mathrm{w} \\
(-) 0.60\end{array}$ & $\begin{array}{l}(0.28) 1.50 \mathrm{~A}^{2} \mathrm{ur} \\
(0.69) 1.20 \mathrm{~B} \mathrm{ur} \\
(0.20) 0.20,0.60\end{array}$ & $\begin{array}{l}-1 \\
-1\end{array}$ & $\begin{array}{l}-1 \\
-1\end{array}$ \\
\hline $\begin{array}{r}4,492.42 \\
4,505.95 \\
13.58 \\
14.02 \\
4,522.05\end{array}$ & $\begin{array}{l}3 \\
25 \\
3 \\
5 \\
2\end{array}$ & $\begin{array}{l}4 \text { III } \\
50 \text { II } \\
4 \text { III } \\
8 \text { III } \\
3 \text { III }\end{array}$ & $\begin{array}{r}253.5 \\
186.7 \\
149.3 \\
147.0 \\
22,107.7\end{array}$ & $\begin{array}{l}z^{2} \mathrm{P}_{2}-b^{2} \mathrm{P}_{1}^{\prime} \\
a^{4} F^{\prime}{ }_{3}-y^{4} \mathrm{D}^{\prime}{ }_{2} \\
a^{2} \mathrm{~F}^{\prime}{ }_{3}-y^{4} \mathrm{P}_{3} \\
a^{4} \mathrm{P}_{2}^{\prime}-y^{4} \mathrm{P}_{3} \\
z^{4} \mathrm{D}_{1}^{\prime} b^{4} \mathrm{D}_{2}\end{array}$ & $\begin{array}{l}\text { (-) } 1.48 \\
(0.00) 0.90 \\
(-) 1.24 \\
(-) 1.31 \\
(-) 1.79\end{array}$ & 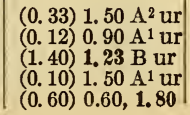 & $\begin{array}{l}-2 ? \\
-1 ?\end{array}$ & $\begin{array}{r}-2 \\
0\end{array}$ \\
\hline
\end{tabular}


TABLE 6.-Classified lines of $Y t I$-Continued

\begin{tabular}{|c|c|c|c|c|c|c|c|c|}
\hline \multirow{2}{*}{$\lambda$} & \multirow{2}{*}{$\begin{array}{l}\text { Intensity } \\
\text { IM }\end{array}$} & \multirow{2}{*}{$\begin{array}{l}\text { Intensity } \\
\text { Class K }\end{array}$} & \multirow{2}{*}{$\nu$} & \multirow{2}{*}{ Terms } & \multicolumn{2}{|c|}{ Zeeman effect } & \multicolumn{2}{|c|}{ Solar intensity } \\
\hline & & & & & Observed & Calculated & Disk & Spot \\
\hline $\begin{array}{r}4,527.25 \\
27.80 \\
28.10 \\
33.50 \\
34.09\end{array}$ & $\begin{array}{l}40 \\
30 \\
2 \\
1 \\
3\end{array}$ & $\begin{array}{l}80 \text { II } \\
50 \text { II } \\
3 \text { III } \\
1 \text { IV } \\
3 \text { III }\end{array}$ & $\begin{array}{r}22,082.3 \\
079.6 \\
078.1 \\
051.8 \\
049.0\end{array}$ & $\begin{array}{l}a^{4} \mathrm{~F}^{\prime}{ }_{5}-y^{4} \mathrm{D}^{\prime}{ }_{4} \\
a^{4} \mathrm{~F}^{\prime}{ }_{4-y^{4}} \mathrm{D}^{\prime}{ }_{3} \\
z^{4} \mathrm{D}^{\prime}{ }_{2}-b^{4} \mathrm{D}_{3} \\
z^{2} \mathrm{P}_{2}-a^{4} \mathrm{D}_{3} \\
z^{4} \mathrm{D}^{\prime}{ }_{3}-b^{4} \mathrm{D}_{4}\end{array}$ & $\begin{array}{l}(0.00) 1.15 \mathrm{~A}^{1} \\
(0.00) 1.05 \mathrm{~A}^{1}\end{array}$ & $\begin{array}{l}(0.12) 1.17 \mathrm{~A}^{1} \mathrm{ur} \\
(0.12) 1.07 \mathrm{~A}^{1} \mathrm{ur}\end{array}$ & $\underset{\mathrm{m}}{\mathrm{m}}$ & $\underset{\mathrm{m}}{\mathrm{m}}$ \\
\hline $\begin{array}{l}35.31 \\
37.16 \\
39.60 \\
44.31 \\
5 \frac{1}{2} .46\end{array}$ & $\begin{array}{l}1 \\
2 \\
1 \\
6 \\
2\end{array}$ & $\begin{array}{l}2 \text { III } \\
1 \text { III } \\
10 \text { III } \\
2 \text { III }\end{array}$ & $\begin{array}{r}038.2 \\
034.0 \\
22,022.2 \\
21,999.4 \\
950.4\end{array}$ & $\begin{array}{l}2^{4} \mathrm{D}^{\prime}{ }^{\prime}-a^{4} \mathrm{G}_{3} \\
2^{4} \mathrm{D}_{1}^{\prime}{ }_{1}^{4} \mathrm{D}_{1} \\
a^{4} \mathrm{P}^{\prime}{ }_{1}-w^{2} \mathrm{P}_{2} \\
a^{4} \mathrm{P}^{\prime}{ }^{\prime}-y^{4} \mathrm{P}_{3} \\
a^{4} \mathrm{P}_{2}^{\prime}{ }_{2}-w^{2} \mathrm{P}_{1}\end{array}$ & . & in & & \\
\hline $\begin{array}{l}55.30 \\
59.37 \\
61.8\end{array}$ & $\begin{array}{l}2 \\
7 \\
2\end{array}$ & $10 \mathrm{III}$ & $\begin{array}{l}946.3 \\
926.7 \\
915.0\end{array}$ & $\begin{array}{l}z^{4} \mathrm{D}^{\prime}{ }_{2}-b^{4} \mathrm{D}_{2} \\
a^{4} \mathrm{P}^{\prime}{ }_{1}-y^{4} \mathrm{P}_{2} \\
a^{4} \mathrm{P}_{2}^{\prime}-w^{2} \mathrm{P}_{2}\end{array}$ & $(-) 1.03,2.04$ & $(0.47) 1.26,2.20$ & $-3 ?$ & \\
\hline $\begin{array}{l}64.94 \\
70.67\end{array}$ & $\begin{array}{l}2 \\
2\end{array}$ & $\begin{array}{l}2 \text { IV? } \\
\text { 3n III }\end{array}$ & $\begin{array}{l}900.0 \\
872.5\end{array}$ & $\begin{array}{l}a^{4} F^{\prime}{ }_{5}-z^{2} G^{\prime}{ }_{4} \\
z^{4} \mathrm{D}_{2}^{\prime}{ }_{2}-b^{4} \mathrm{D}_{1}\end{array}$ & & & & \\
\hline $\begin{array}{l}73.56 \\
78.87 \\
81.32\end{array}$ & $\begin{array}{l}6 \\
1 \\
2\end{array}$ & $\begin{array}{l}10 \text { III } \\
1 \text { III } \\
6 \text { II A }\end{array}$ & $\begin{array}{l}858.7 \\
833.3 \\
821.7\end{array}$ & $\begin{array}{l}z^{4} \mathrm{D}^{\prime}{ }_{3}-b^{4} \mathrm{D}_{3} \\
z^{4} \mathrm{D}^{\prime}{ }_{4}-a^{4} \mathrm{G}_{5} \\
a^{2} F^{\prime}{ }_{3}-y^{4} \mathrm{P}_{2}\end{array}$ & $(-) 1.20$ & $(0.00) 1.38$ & & \\
\hline 81.77 & 1 & $3 \operatorname{III} \mathrm{A}$ & 819.5 & $\left\{\begin{array}{l}a^{4} \mathrm{P}^{\prime}{ }_{2}-y^{4} \mathrm{P}_{2} \\
z^{4} \mathrm{D}^{\prime}{ }_{3}-a^{4} \mathrm{G}_{3} ?\end{array}\right.$ & & 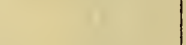 & & \\
\hline 82.16 & 1 & $2 \mathrm{III}$ & 817.7 & $a^{4} \mathrm{P}_{1}^{\prime}-y^{4} \mathrm{P}_{1}$ & & & & \\
\hline $\begin{array}{r}4,596.55 \\
4,601.28 \\
04.80 \\
13.00 \\
36.50\end{array}$ & $\begin{array}{l}10 \\
1 \\
6 \\
2 \\
(1 \mathrm{E} \& \mathrm{H})\end{array}$ & $\begin{array}{l}12 \text { III } \\
2 \text { III } \\
10 \text { III } \\
4 \text { III } \\
1 \text { IV }\end{array}$ & $\begin{array}{l}749.4 \\
727.0 \\
710.4 \\
671.8 \\
562.0\end{array}$ & $\begin{array}{l}z^{4} \mathrm{D}^{\prime}{ }^{\prime}-b^{4} \mathrm{D}_{4} \\
z^{4} \mathrm{D}^{\prime}{ }_{3}-b^{4} \mathrm{D}_{2} \\
a^{4} \mathrm{P}^{\prime}{ }^{2}{ }^{4} y^{4} \mathrm{P}_{1} \\
a^{4} \mathrm{P}^{\prime}{ }^{3}-y^{4} \mathrm{P}_{2} \\
z^{2} \mathrm{P}_{1}-b^{4} \mathrm{P}_{2}^{\prime}{ }_{2}\end{array}$ & $\begin{array}{l}(-) 1.34 \\
(-) 1.26\end{array}$ & $\begin{array}{l}(0.00) 1.43 \\
(0.47) 1.26,2.20\end{array}$ & -1 & -1 \\
\hline $\begin{array}{l}43.69 \\
52.13 \\
53.78 \\
54.47 \\
56.03\end{array}$ & 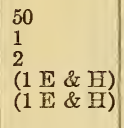 & $\begin{array}{l}150 \mathrm{I} \\
4 \mathrm{III}\end{array}$ & $\begin{array}{l}528.6 \\
489.5 \\
481.9 \\
478.7 \\
471.5\end{array}$ & $\begin{array}{l}a^{2} \mathrm{D}_{2}-z^{2} \mathrm{~F}_{3} \\
z^{4} \mathrm{P}_{2}-d^{4} \mathrm{P}^{\prime}{ }_{3} \\
b^{2} \mathrm{D}_{2}-y^{4} \mathrm{P}_{3} \\
z^{4} \mathrm{P}_{1}-d^{4} \mathrm{P}^{\prime}{ }_{2} \\
z^{4} \mathrm{~F}_{2}-d^{2} \mathrm{D}_{2}\end{array}$ & $(0.00) 0.89$ & $(0.04) 0.90 \mathrm{~A}^{2} \mathrm{ur}$ & $-I N$ & 2 \\
\hline $\begin{array}{l}58.32 \\
66.38\end{array}$ & $\begin{array}{l}10 \\
1\end{array}$ & $\begin{array}{l}12 \text { III } \\
3 \text { III }\end{array}$ & $\begin{array}{l}461.0 \\
423.9\end{array}$ & $b^{2} \mathrm{D}_{3}-w^{2} \mathrm{~F}_{4}$ & $(0.00) 0.94$ & $(0.06) 1.07 \mathrm{~A}^{1} \mathrm{ur}$ & & \\
\hline 66.84 & 2 & $3 \mathrm{III}$ & 421.8 & $\left\{\begin{array}{l}a^{4} \mathrm{P}^{\prime}{ }_{2}-z^{4} \mathrm{~S}^{\prime}{ }_{2} \\
z^{2} \mathrm{~F}_{4}-d^{4} \mathrm{~F}^{\prime}{ }_{3}\end{array}\right.$ & & & & \\
\hline $\begin{array}{l}67.47 \\
71.87\end{array}$ & $(1 \mathrm{E} \& \mathrm{H})$ & 8 III & $\begin{array}{l}418.9 \\
398.7\end{array}$ & $a^{2} \mathrm{P}_{1}-v^{2} \mathrm{D}_{2}^{\prime}$ & $(-) 0.84$ & $(0.07) 0.90 \mathrm{~A}^{2} \mathrm{ur}$ & & \\
\hline $\begin{array}{l}74.84 \\
78.35 \\
89.77 \\
91.97 \\
96.81\end{array}$ & $\begin{array}{l}45 \\
2 \\
3 \\
(1 \mathrm{E}) \\
6\end{array}$ & $\begin{array}{l}125 \text { I } \\
3 \text { III } \\
4 \text { III } \\
1 \text { III A } \\
8 \text { III }\end{array}$ & $\begin{array}{l}385.1 \\
369.1 \\
317.1 \\
307.1 \\
285.1\end{array}$ & $\begin{array}{l}a^{2} \mathrm{D}_{3}-z^{2} \mathrm{~F}_{4} \\
z^{4} \mathrm{P}_{3}-d^{4} \mathrm{P}^{\prime}{ }_{3} \\
b^{2} \mathrm{D}_{3}-y^{4} \mathrm{P}_{3} \\
z^{4} \mathrm{P}_{3}-d^{4} \mathrm{P}^{\prime}{ }^{2} \\
b^{2} \mathrm{D}_{2}-w^{2} \mathrm{P}_{1}\end{array}$ & $\begin{array}{l}(0.00) 1.06 \\
(0.00) 0.97\end{array}$ & $(0.07) 0.73,0.87$ & -2 & 1 \\
\hline $\begin{array}{r}4,699.24 \\
4,701.00 \\
04.64 \\
08.84 \\
18.95\end{array}$ & $\begin{array}{l}(2 \mathrm{E}) \\
1 \\
(1 \mathrm{E}) \\
1 \\
(1 \mathrm{E} \& \mathrm{H})\end{array}$ & $\begin{array}{l}1 \text { III? } \\
2 \text { III } \\
3 \text { III ? }\end{array}$ & $\begin{array}{l}274.1 \\
266.2 \\
249.7 \\
230.7 \\
185.3\end{array}$ & $\begin{array}{l}a^{4} \mathrm{P}^{\prime}{ }_{3}-z^{4} \mathrm{~S}^{\prime}{ }_{2} \\
a^{2} \mathrm{P}^{\prime}{ }_{2}-v^{2} \mathrm{D}^{\prime}{ }_{3} \\
b^{2} \mathrm{D}_{2}-w^{2} \mathrm{P}_{2} \\
a^{4} \mathrm{P}^{\prime}{ }_{1}-w^{2} \mathrm{D}^{\prime}{ }_{2} \\
z^{4} \mathrm{~F}_{3}-d^{2} \mathrm{D}_{3}\end{array}$ & & 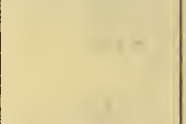 & & \\
\hline 25.84 & 2 & 3 III & 154.4 & $\left\{\begin{array}{l}z^{2} \mathrm{~F}_{4}-e^{2} \mathrm{D}_{3} ? \\
b^{2} \mathrm{D}_{2}-y^{4} \mathrm{P}_{2}\end{array}\right.$ & & & & \\
\hline $\begin{array}{l}28.52 \\
32.37 \\
41.40 \\
52.79\end{array}$ & $\begin{array}{l}10 \\
3 \\
5 \\
10\end{array}$ & $\begin{array}{l}20 \text { III? } \\
3 \text { III? } \\
8 \text { III } \\
12 \text { III }\end{array}$ & $\begin{array}{l}142.4 \\
125.2 \\
084.9 \\
034.4\end{array}$ & $\begin{array}{l}z^{2} \mathrm{P}_{1}-a^{2} \mathrm{~S}_{1} \\
a^{2} \mathrm{~F}_{3}^{\prime}-w^{2} \mathrm{D}_{2}^{\prime} \\
b^{2} \mathrm{D}_{3}-w^{2} \mathrm{P}_{2} \\
a^{2} \mathrm{~F}^{\prime}{ }_{3}-x^{4} \mathrm{D}_{4}^{\prime}\end{array}$ & $\begin{array}{l}(0.67) 1.29 \\
(0.00) 1.04 \\
(0.00) 1.90 \mathrm{~A}^{2} \mathrm{w}\end{array}$ & $\begin{array}{l}(0.67) 1.33 \\
(0.10) 1.10 \mathrm{~A}^{1} \mathrm{ur} \\
(0.19+) 2.14 \mathrm{~A}^{2} \\
\mathrm{ur}\end{array} \mid$ & $\mathrm{m}$ & \\
\hline 59.07 & $(1 \mathrm{E} \& \mathrm{H})$ & & $21,006.6$ & $z^{2} \mathrm{P}_{2}-b^{4} \mathrm{P}_{3}^{\prime}$ & {$[(0.50,0.80)$} & $(0.17,0.51,0.86)$ & - & 0 \\
\hline 60.98 & 30 & $40 \mathrm{I}$ & $20,998.2$ & $a^{2} \mathrm{D}_{3}-z^{2} \dot{\mathrm{F}}_{3}$ & $\left\{\begin{array}{l}0.40,0.72,1.04 \\
1.36,1.69\end{array}\right.$ & $\begin{array}{l}0.34,0.69,1.03, \\
1.37,1.72\end{array}$ & & \\
\hline $\begin{array}{l}62.99 \\
80.18 \\
81.04\end{array}$ & $\begin{array}{l}1(\mathrm{E} \& \mathrm{H}) \\
(1 \mathrm{E}) \\
10\end{array}$ & $\begin{array}{l}1 \mathrm{III} \\
10 \mathrm{III}\end{array}$ & $\begin{array}{l}989.4 \\
913.9 \\
910.1\end{array}$ & $\begin{array}{l}b^{2} \mathrm{D}_{3}-y^{4} \mathrm{P}_{2} \\
a^{4} \mathrm{P}^{\prime}{ }^{\prime}-x^{4} \mathrm{D}^{\prime}{ }_{2} \\
a^{4} \mathrm{~F}^{\prime}{ }_{4}-y^{4} \mathrm{~F}_{6}\end{array}$ & $(0.00) 1.45 \mathrm{~A}^{2}$ & $(0.12) 1.50 \mathrm{~A}^{2} \mathrm{ur}$ & $-3 ?$ & \\
\hline $\begin{array}{r}86.89 \\
4,799.30 \\
4,804.31 \\
04.81\end{array}$ & $\begin{array}{l}10 \\
15 \\
3 \\
5\end{array}$ & $\begin{array}{l}10 \text { III } \\
15 \text { III } \\
4 \text { III } \\
6 \text { III }\end{array}$ & $\begin{array}{l}884.6 \\
830.5 \\
808.8 \\
806.7\end{array}$ & $\begin{array}{l}a^{4} \mathrm{P}^{\prime}{ }_{3}-x^{4} \mathrm{D}^{\prime}{ }_{4} \\
a^{4} \overline{\mathrm{F}}^{\prime}{ }_{3}-y^{4} \mathrm{~F}_{4} \\
a^{2} \mathrm{~F}^{\prime}{ }_{3}-x^{4} \mathrm{D}_{2}^{\prime}{ }_{2} \\
a^{4} \mathrm{P}^{\prime}{ }_{2}-x^{4} \mathrm{D}_{2}^{\prime}\end{array}$ & (w) $1.52 \mathrm{~A}^{2}$ & $(0.20) 1.50 \mathrm{~A}^{2} \mathrm{ur}$ & & \\
\hline $4,819.64$ & 7 & 10 III & $20,742.7$ & $a^{4} \mathrm{~F}^{\prime}{ }_{2}-y^{4} \mathrm{~F}_{3}$ & $\left\{\begin{array}{l}(0.31,0.95) \\
0.64,1.28,1.93\end{array}\right.$ & $\begin{array}{l}(0.31,0.94) 0.09 \\
0.71,1.34,1.97\end{array}$ & $-3 ?$ & \\
\hline
\end{tabular}


TABLE 6.-Classified lines of $Y t I$-Continued

\begin{tabular}{|c|c|c|c|c|c|c|c|c|}
\hline \multirow{2}{*}{$\lambda$} & \multirow{2}{*}{$\underset{\mathrm{M}}{\text { Intensity }}$} & \multirow{2}{*}{$\begin{array}{c}\text { Intensity } \\
\text { Class K }\end{array}$} & \multirow{2}{*}{$\nu$} & \multirow{2}{*}{ Terms } & \multicolumn{2}{|c|}{ Zeeman effect } & \multicolumn{2}{|c|}{ Solar intensity } \\
\hline & & & & & Observed & Calculated & Disk & Spot \\
\hline $\begin{array}{r}4,821.62 \\
22.12\end{array}$ & $\begin{array}{l}1 \\
6\end{array}$ & $\begin{array}{l}2 \mathrm{IV} \\
8 \mathrm{III}\end{array}$ & $\begin{array}{l}734.1 \\
732.0\end{array}$ & $\begin{array}{l}a^{2} \mathrm{~F}^{\prime}{ }_{3}-x^{4} \mathrm{D}^{\prime}{ }_{3} \\
a^{4} \mathrm{P}^{\prime}{ }_{2}-x^{4} \mathrm{D}^{\prime}{ }_{3}\end{array}$ & (w) 0.90 & $(0.27) 1.10 \mathrm{~A}^{1} \mathrm{ur}$ & & \\
\hline 39.14 & 2 & 3 III & 659.3 & $a^{4} \mathrm{P}_{3}^{\prime}-x^{4} \mathrm{D}_{2}^{\prime}$ & $(0.34,0.96)-$ & $\{(0.20,0.60) 1.00$, & & \\
\hline $\begin{array}{l}39.87 \\
45.67\end{array}$ & $\begin{array}{l}60 \\
50\end{array}$ & $\begin{array}{l}60 \mathrm{II} \\
50 \mathrm{II}\end{array}$ & $\begin{array}{l}656.0 \\
631.2\end{array}$ & $\begin{array}{l}a^{4} F^{\prime}{ }^{\prime}-y^{4} F_{5} \\
a^{4} F_{4}^{\prime}{ }_{4}-y^{4} F_{4}\end{array}$ & $\begin{array}{l}(0.00) 1.30 \\
(0.00) 1.20\end{array}$ & $\begin{array}{l}(0.00) 1.33 \\
(0.00) 1.24\end{array}$ & $m^{-2}$ & $\bar{m}^{-1}$ \\
\hline $\begin{array}{l}52.69 \\
54.28\end{array}$ & $\begin{array}{l}40 \\
1\end{array}$ & $\begin{array}{l}50 \text { II } \\
4 \text { III }\end{array}$ & $\begin{array}{l}601.4 \\
594.6\end{array}$ & $\begin{array}{l}a^{4} \mathrm{~F}^{\prime}{ }^{\prime}-y^{4} \mathrm{~F}_{3} \\
a^{4} \mathrm{P}^{\prime}{ }_{1}-x^{4} \mathrm{D}^{\prime}{ }^{2}\end{array}$ & & $(0.00) 1.03$ & - & -2 \\
\hline $\begin{array}{l}56.70 \\
59.84 \\
79.64\end{array}$ & $\begin{array}{l}2 \\
25 \\
(2 \mathrm{E})\end{array}$ & $\begin{array}{l}3 \text { III } \\
40 \text { II } \\
4 \text { III }\end{array}$ & $\begin{array}{l}584.4 \\
571.1 \\
487.6\end{array}$ & $\begin{array}{l}a^{4} \mathbf{P}^{\prime}{ }^{3}-x^{4} D^{\prime}{ }^{4} \\
a^{4} F^{\prime}{ }^{2}-y^{4} F_{2} \\
a^{4} P^{\prime}{ }_{2}-x^{4} D^{\prime}{ }_{1}\end{array}$ & $\begin{array}{l}\text { (w?) } 1.28 \\
(0.00) 0.40 \\
(0.70) 2.28\end{array}$ & $\begin{array}{l}(0.45) 1.48 \mathrm{~B} \text { ur } \\
(0.00) 0.40 \\
(0.81) 0.81,2.60\end{array}$ & $\mathrm{~m}$ & $\mathrm{~m}$ \\
\hline $\begin{array}{l}86.28 \\
86.65\end{array}$ & $\begin{array}{l}3 \\
3\end{array}$ & $\begin{array}{l}4 \text { III } \\
3 \text { III }\end{array}$ & $\begin{array}{l}459.8 \\
458.2\end{array}$ & $\begin{array}{l}b^{2} \mathrm{D}_{3}-w^{2} \mathrm{D}_{3}^{\prime} \\
b^{2} \mathrm{D}_{2}-w^{2} \mathrm{D}_{2}^{\prime}\end{array}$ & $\begin{array}{l}(0.00) 1.24 \\
(0.00) \\
0.84\end{array}$ & $\begin{array}{l}(0.00) 1.20 \\
(0.00) \\
0.80\end{array}$ & & \\
\hline $4,893.44$ & 8 & 6 III A & 429.8 & $a^{4} F^{\prime}{ }_{3}-y^{4} F_{2}$ & $\left\{\begin{array}{l}(0.32,0.95) \\
0.70,1.30,1.90\end{array}\right.$ & $\begin{array}{c}(0.31,0.94) 0.09 \\
0.71,1.34,1.97\end{array}$ & & \\
\hline $\begin{array}{l}06.11 \\
09.00\end{array}$ & $\begin{array}{l}8 \\
2\end{array}$ & $\begin{array}{l}6 \text { III A } \\
2 \text { III }\end{array}$ & $\begin{array}{l}377.1 \\
365.1\end{array}$ & $\begin{array}{l}a^{4} \mathrm{~F}^{\prime}{ }_{5}-y^{4} \mathrm{~F}_{4} \\
z^{2} \mathrm{D}^{\prime}{ }_{3}-d^{2} \mathrm{D}_{3}\end{array}$ & $\begin{array}{l}(0.00) 1.50 \mathrm{~A}^{2} \\
(0.00) 1.18\end{array}$ & $\begin{array}{l}(0.12) 1.50 \AA^{2} \text { ur } \\
(0.00) 1.20\end{array}$ & & \\
\hline $\begin{array}{l}21.86 \\
26.31\end{array}$ & $\begin{array}{l}10 \\
1\end{array}$ & $\begin{array}{l}10 \text { III A } \\
\text { tr III A }\end{array}$ & $\begin{array}{l}311.8 \\
293.5\end{array}$ & $\begin{array}{l}{ }^{2} \mathrm{P}_{2}-a^{2} \mathrm{~S}_{1} \\
b^{2} \mathrm{D}_{3}-w^{2} \mathrm{D}_{2}^{\prime}\end{array}$ & $(0.35) 0.95,1.66$ & $(0.33) 1.00,1.67$ & & \\
\hline 28.22 & 2 & $1 \mathrm{III}$ & 285.6 & $\left\{\begin{array}{l}z^{2} \mathrm{D}_{2}^{\prime}-d^{2} \mathrm{D}_{3} \\
y^{2} \mathrm{P}_{1}-(2,1)\end{array}\right.$ & & & & \\
\hline $\begin{array}{l}30.94 \\
48.54\end{array}$ & $\begin{array}{l}3 \\
1\end{array}$ & $\underset{t r I V}{2 \text { III }}$ & $\begin{array}{l}274.5 \\
202.4\end{array}$ & $\begin{array}{l}z^{2} \mathrm{D}_{2}^{\prime}{ }_{2}-d^{2} \mathrm{D}_{2} \\
b^{2} \mathrm{D}_{3}-x^{4} \mathrm{D}_{4}^{\prime}\end{array}$ & $(0.00) 0.83$ & $(0.00) 0.80$ & & \\
\hline $\begin{array}{r}74.30 \\
4,977.54 \\
5,003.67 \\
06.96 \\
70.20\end{array}$ & $\begin{array}{l}6 \\
2\end{array}$ & $\begin{array}{l}10 \mathrm{III} ? \\
(0 \mathrm{H} \mathrm{N} \mathrm{R}) \\
(0 \mathrm{H} \mathrm{N} \mathrm{R}) \\
10 \mathrm{IV} \\
5 \mathrm{~V}\end{array}$ & $\begin{array}{r}097.7 \\
20,084.7 \\
19,979.8 \\
966.6 \\
717.6\end{array}$ & $\begin{array}{l}a^{2} G_{5}-y^{2} G_{5}^{\prime} \\
a^{2} G_{4}-y^{2} G_{5}^{\prime} \\
a^{2} G_{5}-y^{2} G_{4}^{\prime} \\
a^{2} G_{4}-y^{2} G_{4}^{\prime} \\
z^{4} P_{3}-b^{4} D_{4}\end{array}$ & $\begin{array}{l}(0.00) 1.08 \\
(0.00) 0.87\end{array}$ & $\begin{array}{l}(0.00) 1.11 \\
(0.00) 0.89\end{array}$ & & \\
\hline $5,088.18$ & 1 & $3 \mathrm{~V}$ & $\begin{array}{r}647.9 \\
493.8\end{array}$ & ${ }_{24}^{4} \mathrm{P}_{2}-b^{4} \mathrm{D}_{3}$ & & & & \\
\hline $\begin{array}{r}5,135.20 \\
5,228.56 \\
37.20\end{array}$ & $\begin{array}{l}1 \\
4 \\
2\end{array}$ & $\begin{array}{l}25 \mathrm{III} \\
(0 \mathrm{H} N \mathrm{~N})\end{array}$ & $\begin{array}{l}493.8 \\
468.0 \\
120.4 \\
088.9\end{array}$ & $\begin{array}{l}a^{2} \mathrm{~F}_{5}-z^{2} \mathrm{U}_{6} \\
a^{2} \mathrm{G}_{5}-2^{2} \mathrm{~F}_{4} \\
a^{2} \mathrm{G}_{5}-z^{2} \mathrm{H}_{5}\end{array}$ & $(0.00) 1.02$ & $(0.02) 1.04 \mathrm{~A}^{1} \mathrm{ur}$ & & \\
\hline $\begin{array}{r}40.80 \\
5,283.68 \\
5,325.84 \\
69.78\end{array}$ & $\begin{array}{l}4 \\
2 \\
1\end{array}$ & $15 \mathrm{IV}$ & $\begin{array}{r}19,075.8 \\
18,921.0 \\
771.2 \\
617.6\end{array}$ & $\begin{array}{l}a^{2} \mathrm{G}_{4}-z^{2} \mathrm{H}_{5} \\
a^{2} \mathrm{~F}^{\prime}{ }_{3}-x^{2} \mathrm{D}_{3}^{\prime} \\
a^{4} \mathrm{P}_{3}^{\prime} x_{3}^{2} \mathrm{D}_{3}^{\prime} \\
a^{2} \mathrm{D}_{3}-z^{4} \mathrm{P}_{3}\end{array}$ & $(0.00) 0.94$ & $(0.02) 0.94 \mathrm{~A}^{2} \mathrm{ur}$ & & \\
\hline 75.87 & 1 & & 596.5 & $z^{4} \mathrm{~F}_{3}-b^{2} \mathrm{P}_{2}^{\prime}$ & & & & \\
\hline $\begin{array}{r}80.62 \\
88.41 \\
5,390.82 \\
5,417.02\end{array}$ & $\begin{array}{l}5 \\
1 \\
2 \\
2\end{array}$ & $3 \mathrm{IV} ?$ & $\begin{array}{l}580.1 \\
553.2 \\
545.0 \\
455.2\end{array}$ & $\begin{array}{l}a^{2} F^{\prime}{ }_{3}-x^{2} D^{\prime}{ }_{2} \\
a^{4} \mathrm{P}^{\prime}{ }_{3}-x^{2} \mathrm{~F}_{4} \\
z^{4} \mathrm{~F}_{4}-c^{2} \mathrm{D}_{3} \\
z^{2} \mathrm{D}_{3}^{\prime}{ }_{3}-c^{4} \mathrm{P}_{3}^{\prime}\end{array}$ & & 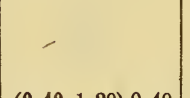 & & \\
\hline 24. 36 & 5 & $20 \mathrm{III}$ & 430.2 & $a^{4} \mathrm{P}_{3}^{\prime}-x^{2} \mathrm{D}_{2}^{\prime}$ & $\left\{\begin{array}{l}(0.22,0.66) \text { 䖝 } \\
1.78 \mathrm{~A}^{2}\end{array}\right.$ & $\begin{array}{c}(0.40,1.20) 0.40 \\
1.20,2.00,2.89\end{array}$ & & \\
\hline 38.24 & 20 & $50 \mathrm{II}$ & 383.2 & $a^{2}{F^{\prime}}_{4}{ }^{2}-x^{2} \mathrm{D}^{\prime}{ }_{3}$ & $(0.00) 1.09 \mathrm{~B} ?$ & $(0.06) 1.07 \mathrm{~A}^{1} \mathrm{ur}$ & & \\
\hline 66.46 & 50 & $300 \mathrm{II}$ & 288.3 & $\left\{\begin{array}{l}a^{4} \mathrm{~F}^{\prime} \sigma^{4} \mathrm{G}^{\prime} 6 \\
z^{4} \mathrm{~F}_{2}-a^{4} \mathrm{D}_{2}\end{array}\right.$ & $(0.00) 1.10$ & $(0.09) 1.16 \mathrm{~A}^{1} \mathrm{ur}$ & $\mathrm{m}$ & $\mathrm{m}$ \\
\hline $\begin{array}{r}68.47 \\
69.19 \\
91.45 \\
93.16 \\
5,495.59 \\
5,503.34 \\
03.47 \\
13.66\end{array}$ & $\begin{array}{l}10 \\
1 \\
(3 \mathrm{E}) \\
5 \\
2 \\
2 \\
10 \\
5\end{array}$ & $\begin{array}{l}40 \text { III } \\
1 \text { V } \\
15 \text { III } \\
8 \text { III } \\
100\left\{\begin{array}{l}5 \text { III } \\
25 \text { II } \\
20 \text { II }\end{array}\right.\end{array}$ & $\begin{array}{l}281.6 \\
279.2 \\
205.1 \\
199.4 \\
191.4 \\
165.8 \\
165.4 \\
131.8\end{array}$ & $\begin{array}{l}a^{8} \mathrm{~F}^{\prime}{ }_{3}-x^{2} \mathrm{~F}_{3} \\
a^{4} \mathrm{P}^{\prime}{ }_{2}-x^{2} \mathrm{~F}_{3} \\
y^{2} \mathrm{P}_{2}-b^{2} \mathrm{~S}_{1} \\
z^{4} \mathrm{~F}_{3}-a^{4} \mathrm{D}_{1} \\
z^{2} \mathrm{D}^{\prime}{ }_{3}-c^{2} \mathrm{D}_{3} \\
z^{4} \mathrm{~F}_{3}-a^{4} \mathrm{D}_{3} \\
a^{2} \mathrm{~F}^{\prime}{ }_{1}-x^{2} \mathrm{~F}_{4} \\
a^{4} \mathrm{P}^{\prime}{ }_{3}-x^{2} \mathrm{~F}_{3}\end{array}$ & $\begin{array}{l}(0.39) 1.04 \\
(0.22) 0.62 \\
(0.00) 1.15 \\
(0.65)-\end{array}$ & $\begin{array}{l}(0.00) 0.86 \\
(0.20) 0.20,0,60 \\
(0.00) 1.14\end{array}$ & & \\
\hline $\begin{array}{l}21.59 \\
26.74\end{array}$ & 10 & $\begin{array}{l}30 \mathrm{II}+\mathrm{E} \\
2 \mathrm{IV}\end{array}$ & $\begin{array}{l}105.7 \\
088.8\end{array}$ & $\begin{array}{l}a^{2} \mathrm{~F}_{3}^{\prime}-z^{2} \mathrm{G}^{\prime}{ }_{4} \\
b^{2} \mathrm{D}_{3}-x^{2} \mathrm{D}_{3}^{\prime}\end{array}$ & & & $-1 \mathrm{~m}$ & $\mathrm{~m}$ \\
\hline $\begin{array}{l}27.54 \\
27.75 \\
40.61\end{array}$ & $\begin{array}{l}40 \\
(1 \mathrm{E})\end{array}$ & 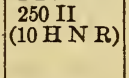 & $\begin{array}{l}086.2 \\
085.5 \\
043.6\end{array}$ & $\begin{array}{l}a^{4} \mathrm{~F}^{\prime}{ }^{\prime}-z^{4} \mathrm{G}^{\prime}{ }_{5} \\
z^{2} \mathrm{D}^{\prime}{ }^{\prime} 2-c^{2} \mathrm{D}_{2} \\
a^{4} \mathrm{P}^{\prime}{ }_{1-y^{4} \mathrm{D}^{\prime}{ }_{2}}\end{array}$ & $(0.00) 1.03 \mathrm{~A}^{1}$ & $(0.08) 1.05 \mathrm{~A}^{1} \mathrm{ur}$ & $-2 d b$ & $-1 b$ \\
\hline $\begin{array}{r}41.64 \\
44.61 \\
51.00 \\
56.43 \\
5,567.75\end{array}$ & $\begin{array}{l}2 \\
15 \\
1 \\
5 \\
5\end{array}$ & $\begin{array}{l}3 \mathrm{III} \\
25 \mathrm{III}+\mathrm{E} \\
20 \mathrm{I} \mathrm{A} \\
15 \mathrm{II}\end{array}$ & $\begin{array}{r}040.2 \\
030.5 \\
18,009.8 \\
17,992.2 \\
17,955.6\end{array}$ & $\begin{array}{l}z^{4} \mathrm{~F}_{4}-a^{4} \mathrm{D}_{4} \\
a^{2} \mathrm{~F}^{-} a_{3}-y^{4} \mathrm{D}^{\prime}{ }_{3} \\
z^{2} \mathrm{D}^{\prime}-c^{4} \mathrm{P}^{\prime}{ }_{2} \\
z^{4} \mathrm{~F}_{3}-a^{4} \mathrm{D}_{2} \\
a^{4} \mathrm{P}^{\prime}-z^{2} \mathrm{G}_{4}^{\prime}\end{array}$ & $(0.00) 0.09$ & $(0.12) 0.90 \mathrm{~A}^{1} \mathrm{ur}$ & $\begin{array}{l}-2 \mathrm{~m} \\
-3\end{array}$ & $\begin{array}{l}-1 m \\
-2\end{array}$ \\
\hline
\end{tabular}


TABLE 6.-Classified lines of $Y t I$-Continued

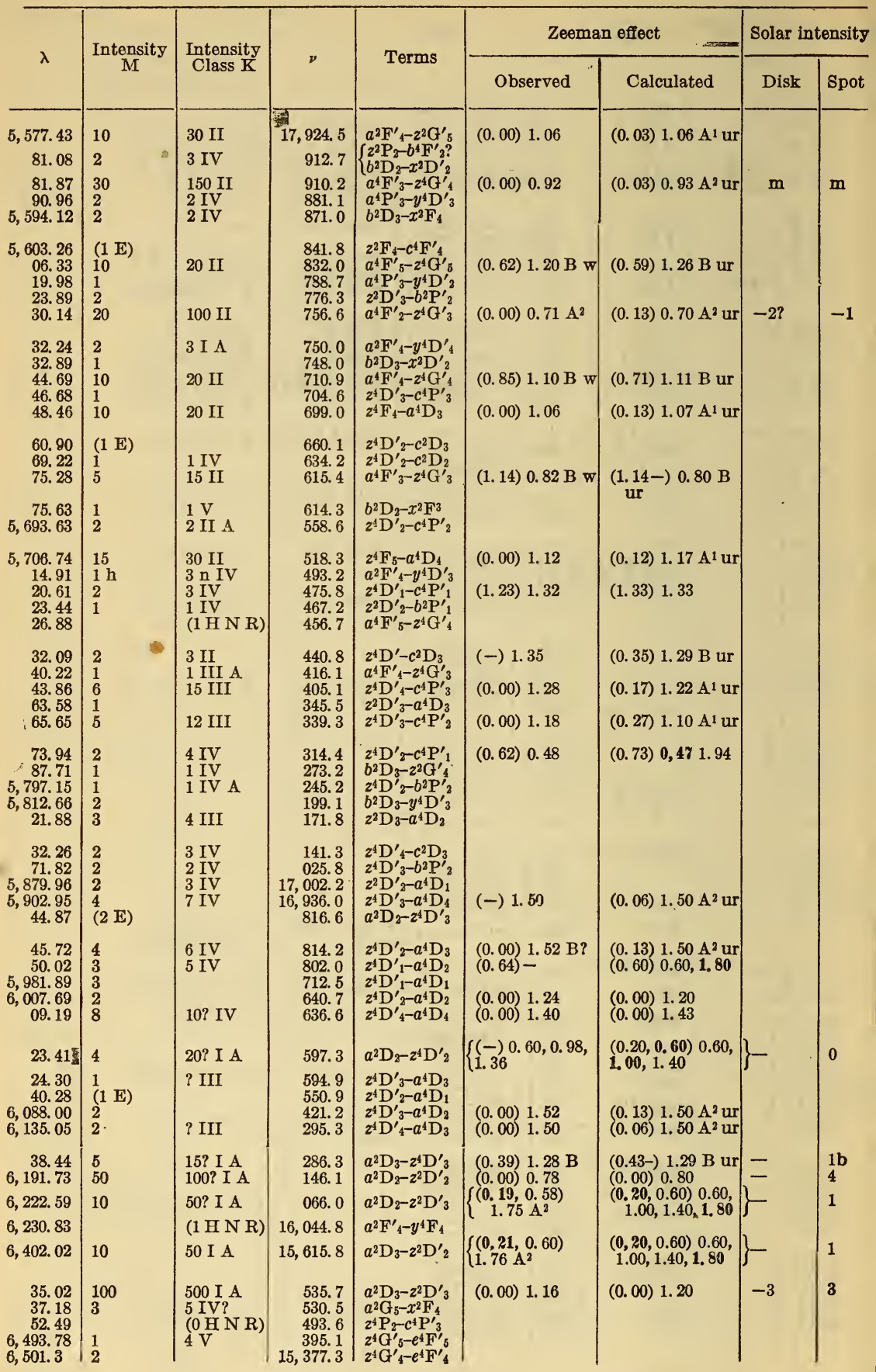




\section{TABLE 6.-Classified lines of $Y t I-$ Continued}

\begin{tabular}{|c|c|c|c|c|c|c|c|c|}
\hline \multirow{2}{*}{$\lambda$} & \multirow{2}{*}{$\begin{array}{c}\text { Intensity } \\
\text { M. }\end{array}$} & \multirow{2}{*}{$\begin{array}{c}\text { Intensity } \\
\text { Class K }\end{array}$} & \multirow{2}{*}{$\nu$} & \multirow{2}{*}{ Terms } & \multicolumn{2}{|c|}{ Zeeman effect } & \multicolumn{2}{|c|}{ Solar intensity } \\
\hline & & & & & Observed & Calculated & Disk & Spot \\
\hline $\begin{array}{r}6,503.05 \\
33.59 \\
44.19\end{array}$ & 15 & $\begin{array}{l}(0 \mathrm{H} \mathrm{N} \mathrm{R}) \\
35 \text { III } \\
(0 \mathrm{H} \mathrm{N} \mathrm{R})\end{array}$ & $\begin{array}{r}15,373.2 \\
289.6 \\
276.5\end{array}$ & $\begin{array}{l}z^{4} \mathrm{P}_{3}-c^{4} \mathrm{P}^{\prime}{ }_{3} \\
a^{2} \mathrm{G}_{5}-z^{2} \mathrm{G}^{\prime}{ }_{5} \\
a^{2} \mathrm{G}_{4}-z^{2} \mathrm{G}_{5}^{\prime}\end{array}$ & $(0.00) 1.12$ & $(0.00) 1.11$ & $-2 m$ & $-2 m$ \\
\hline 57.40 & 10 & $30 \mathrm{IA}$ & 245.7 & $a^{2} \mathrm{D}_{2}-z^{4} \mathrm{~F}_{3}$ & $(0.00) 1.37 \mathrm{~A}^{2}$ & $\left\{\begin{array}{c}(0.11+) 1.37- \\
A^{2} \text { ur }\end{array}\right.$ & \} & -1 \\
\hline 76.86 & 5 & $6 \mathrm{III}$ & 200.6 & 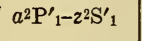 & $(-) 1.40$ & $(0.67) 1.33$ & & \\
\hline $\begin{array}{r}6,584.89 \\
6,595.04 \\
6,616.59 \\
22.50 \\
36.48\end{array}$ & $\begin{array}{l}2 \\
1 \\
(1 \mathrm{E}) \\
1 \\
2\end{array}$ & $\begin{array}{l}5 \text { II A } \\
2 \text { IV? } \\
2 \text { IV? }\end{array}$ & $\begin{array}{l}182.1 \\
158.7 \\
109.4 \\
095.9 \\
064.1\end{array}$ & $\begin{array}{l}a^{2} \mathrm{D}_{3}-z^{4} \mathrm{~F}_{4} \\
z^{2} \mathrm{~F}_{4}-\left(1_{3}\right) \\
z^{4} \mathrm{P}_{3}-c^{2} D_{3} \\
a^{2} \mathrm{G}_{4}-x^{2} \mathrm{~F}_{3} \\
z^{4} G^{\prime}{ }^{-}-e^{4} \mathrm{~F}^{\prime}{ }_{3}\end{array}$ & & & & \\
\hline $\begin{array}{l}50.60 \\
64.38\end{array}$ & $\begin{array}{l}2 \\
2\end{array}$ & $\begin{array}{l}8 \mathrm{IV} \\
3 \mathrm{IV} ?\end{array}$ & $\begin{array}{r}032.1 \\
15,001.0\end{array}$ & $\begin{array}{l}a^{2} \mathrm{P}^{\prime}{ }_{2}-z^{2} \mathrm{~S}^{\prime}{ }_{1} \\
z^{4}{\mathrm{G}^{\prime}}_{5}-e^{4} \mathrm{~F}^{\prime}{ }_{4}\end{array}$ & $(0.20) 1.04$ & (0.33) 1.00, 1.67 & & \\
\hline 87.57 & 25 & $80 \mathrm{I}$ & $14,949.0$ & $a^{2} \mathrm{D}_{2}-z^{4} \mathrm{~F}_{2}$ & $(0.52) 0.63 \mathrm{~B}$ & $\left\{\begin{array}{l}(0.20,0.60) 0.20 \\
0.60,1.00\end{array}\right.$ & \} & 0 \\
\hline $\begin{array}{r}91.82 \\
6,694.75\end{array}$ & $\begin{array}{l}2 \\
1\end{array}$ & $\begin{array}{l}2 \mathrm{~V} \\
1 \mathrm{~V}\end{array}$ & $\begin{array}{l}939.5 \\
933.0\end{array}$ & $\begin{array}{l}z^{4} \mathrm{G}_{6}^{\prime}-e^{4} \mathrm{~F}^{\prime}{ }^{\prime} \\
a^{2} \mathrm{G}_{5}-z^{2} \mathrm{G}^{\prime}{ }_{4}\end{array}$ & & & & \\
\hline $\begin{array}{r}6,700.71 \\
13.20 \\
35.98\end{array}$ & $\begin{array}{l}15 \\
4 \\
5\end{array}$ & $\begin{array}{l}20 \text { III } \\
6 \text { III } \\
7 \text { III }\end{array}$ & $\begin{array}{l}919.7 \\
891.9 \\
841.6\end{array}$ & $\begin{array}{l}a^{2} \mathrm{G}^{4}-z^{2} \mathrm{G}^{\prime}{ }_{4} \\
z^{2} \mathrm{~F}_{3}-d^{2} \mathrm{D}_{2} \\
a^{2} \mathrm{P}_{2}^{\prime}{ }_{2}-x^{2} \mathrm{D}_{3}^{\prime}\end{array}$ & $(0.00) 0.89$ & $(0.00) 0.89$ & & \\
\hline $\begin{array}{l}6,793.71 \\
6,803.14\end{array}$ & $\begin{array}{l}25 \\
1\end{array}$ & $80 \mathrm{I}$ & $\begin{array}{l}715.4 \\
695.0\end{array}$ & $\begin{array}{l}a^{2} \mathrm{D}_{3}-z^{4} \mathrm{~F}_{3} \\
z^{4} \mathrm{P}_{3}-b^{2} \mathrm{P}_{2}^{\prime}\end{array}$ & (-) $1.10 \mathrm{~B}$ & (0.34) $1.11 \mathrm{~B}$ ur & $0 N ?$ & 1 \\
\hline 15. 16 & 2 & $5 \mathrm{IV}$ & 669.1 & $a^{2} \mathrm{P}_{1}^{\prime}-x^{2} \mathrm{D}_{2}^{\prime}$ & & & & \\
\hline $\begin{array}{r}45.24 \\
6,887.23 \\
6,908.26 \\
33.55\end{array}$ & $\begin{array}{l}5 \\
2 \\
2 \\
2\end{array}$ & $\begin{array}{l}10 \text { III } \\
8 \text { IV } \\
2 \text { III } \\
6 \text { II A }\end{array}$ & $\begin{array}{l}604.7 \\
515.6 \\
471.3 \\
418.7\end{array}$ & $\begin{array}{l}z^{4} \mathrm{P}_{3}-a^{4} \mathrm{D}_{4} \\
z^{2} \mathrm{~F}_{4}-d^{2} \mathrm{D}_{3} \\
z^{4} \mathrm{~F}_{2}-b^{4} F^{\prime}{ }_{3} \\
a^{2} \mathrm{D}_{3}-z^{4} F_{2}\end{array}$ & $\begin{array}{l}(-) 1.18 \\
(-) 0.99\end{array}$ & $\begin{array}{l}(0.18) 1.22 \mathrm{~A}^{1} \mathrm{ur} \\
(0.06) 1.07 \mathrm{~A}^{1} \mathrm{ur}\end{array}$ & -2 ? & \\
\hline $\begin{array}{r}41.79 \\
50.32 \\
58.08 \\
6,979.88 \\
7.008 .97\end{array}$ & $\begin{array}{l}1 \\
4 \\
2 \\
4 \\
3\end{array}$ & $\begin{array}{l}8 \mathrm{IV} \\
4 \mathrm{IA} \\
8 \mathrm{~V} ? \\
5 \mathrm{~V}\end{array}$ & $\begin{array}{l}401.5 \\
383.9 \\
367.8 \\
323.0 \\
263.5\end{array}$ & $\begin{array}{l}z^{4} \mathrm{G}^{\prime}{ }^{3}-d^{4} \mathrm{~F}^{\prime}{ }_{2} \\
z^{4} \mathrm{P}_{2}-a^{4} \mathrm{D}_{3} \\
z^{4} \mathrm{~F}_{3}-b^{4} \mathrm{~F}^{\prime}{ }_{4} \\
z^{4} \mathrm{~F}_{2}-b^{4} \mathrm{~F}^{\prime}{ }_{2} \\
z^{4} \mathrm{P}_{3}-a^{4} \mathrm{D}_{3}\end{array}$ & & & & \\
\hline $\begin{array}{r}09.93 \\
35.18 \\
52.94 \\
54.28 \\
7,075.17\end{array}$ & $\begin{array}{l}2 \\
3 \\
4 \\
3 \\
2\end{array}$ & $\begin{array}{l}4 \mathrm{~V} \\
5 \mathrm{IV} \\
10 \mathrm{III} \\
4 \mathrm{~V}\end{array}$ & $\begin{array}{l}261.6 \\
210.3 \\
174.6 \\
171.9 \\
130.0\end{array}$ & $\begin{array}{l}z^{4} \mathrm{P}_{1}-a^{4} \mathrm{D}_{2} \\
z^{4} \mathrm{P}_{2}-a^{4} \mathrm{D}_{2} \\
z^{4} \mathrm{~F}_{3}-b^{4} \mathrm{~F}^{\prime}{ }_{3} \\
z^{4} \mathrm{P}_{1}-a^{4} \mathrm{D}_{1} \\
z^{4} \mathrm{~F}_{4}-b^{4} \mathrm{~F}_{5}^{\prime}\end{array}$ & & t & & \\
\hline $\begin{array}{r}7,127.92 \\
91.66 \\
7,195.95 \\
7,295.10 \\
7,303.2\end{array}$ & $\begin{array}{l}3 \mathrm{~h} ? \\
5 \\
2 \\
2 \\
1\end{array}$ & $\begin{array}{l}2 \mathrm{III} ? \\
10 \mathrm{III}\end{array}$ & $\begin{array}{r}14,025.5 \\
13,901.2 \\
892.9 \\
707.8 \\
688.9\end{array}$ & $\begin{array}{l}z^{4} \mathrm{~F}_{3}-b^{4} \mathrm{~F}^{\prime}{ }^{\prime} \\
z^{4} \mathrm{~F}_{4}-b^{4} \mathrm{~F}^{\prime}{ }_{4}^{4} \\
x^{4} \mathrm{D}_{4}^{\prime}-e^{4} \mathrm{P}^{\prime}{ }_{3} \\
z^{4} \mathrm{~F}_{4}-b^{4} \mathrm{~F}^{\prime}{ }_{3} \\
a^{2} \mathrm{G}_{5}-y^{4} \mathrm{~F}_{5}\end{array}$ & & & & \\
\hline $\begin{array}{r}46.47 \\
7,398.80 \\
7,455.20 \\
72.2\end{array}$ & $\begin{array}{l}10 \\
4 \\
2 \\
2\end{array}$ & $10 \mathrm{III}$ & $\begin{array}{l}608.2 \\
512.0 \\
409.8 \\
379.3\end{array}$ & $\begin{array}{l}z^{4} \mathrm{~F}_{5}-b^{4} \mathrm{~F}^{\prime}{ }_{5} \\
a^{4} \mathrm{P}^{\prime}{ }^{\prime}-z^{4} \mathrm{G}^{\prime}{ }_{4} \\
a^{2} \mathrm{G}_{5}-y^{4} \mathrm{~F}_{4} \\
z^{4} \mathrm{~F}_{5}-b^{4} \mathrm{~F}_{4}^{\prime}\end{array}$ & & & & \\
\hline 78.8 & 2 & & 367.5 & $\left\{\begin{array}{l}a^{2} \mathrm{~F}^{\prime}{ }_{3}-z^{4} \mathrm{G}^{\prime}{ }_{3} \\
a^{4} \mathrm{~F}^{\prime}{ }_{5-y^{2}} \mathrm{~F}_{4}\end{array}\right.$ & & & & \\
\hline \begin{tabular}{r|}
86.4 \\
$7,494.90$ \\
$7,563.13$ \\
$7,622.94$ \\
52.89
\end{tabular} & $\begin{array}{l}1 \\
5 \\
10 \\
5 \\
3\end{array}$ & $5 \mathrm{~V}$ & $\begin{array}{l}353.9 \\
338.7 \\
218.4 \\
114.7 \\
063.4\end{array}$ & $\begin{array}{l}z^{2} \mathrm{D}^{\prime}{ }_{3}-b^{4} \mathrm{~F}^{\prime}{ }_{3}{ }^{2} \\
z^{4} \mathrm{P}_{2}-b^{4} \mathrm{P}^{\prime}{ }_{3} \\
z^{4} \mathrm{P}_{3}-b^{4} \mathrm{P}^{\prime}{ }_{3} \\
z^{4} \mathrm{P}_{1}-b^{4} \mathrm{P}_{2}^{\prime} \\
z^{4} \mathrm{P}_{2}-b^{4} \mathrm{P}_{2}^{\prime}{ }_{2}\end{array}$ & 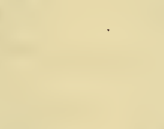 & & & \\
\hline $\begin{array}{r}7,689.49 \\
7,719.89 \\
24.08 \\
88.42 \\
7,796.32\end{array}$ & $\begin{array}{l}2 \\
6 \\
5 \\
3 \\
4\end{array}$ & $2 \mathrm{2} \mathrm{V}$ & $\begin{array}{r}13,001.2 \\
12,950.0 \\
943.0 \\
836.1 \\
823.1\end{array}$ & $\begin{array}{l}z^{4} \mathrm{P}_{1}-b^{4} \mathrm{P}^{\prime}{ }_{1} \\
z^{4} \mathrm{P}_{2}-b^{4} \mathrm{P}_{1}^{\prime}{ }_{1} \\
z^{4} \mathrm{P}_{3}-b^{4} \mathrm{P}_{2}^{\prime} \\
z^{4} \mathrm{D}_{1}^{\prime}{ }_{1}-b^{4} \mathrm{~F}^{\prime}{ }_{2} \\
z^{4} \mathrm{D}_{2}^{\prime}-b^{4} \mathrm{~F}_{3}^{\prime}{ }_{3}\end{array}$ & & & & \\
\hline $\begin{array}{r}7,802.52 \\
12.16 \\
23.94 \\
55.52 \\
7,870.04\end{array}$ & $\begin{array}{l}2 \\
5 \\
1 \\
7 \\
2\end{array}$ & & $\begin{array}{r}812.9 \\
797.0 \\
777.8 \\
726.4 \\
12,702.9\end{array}$ & $\begin{array}{l}a^{2} \mathrm{~F}^{\prime}{ }_{3}-x^{2} \mathrm{P}_{2} \\
z^{4} \mathrm{D}_{3}^{\prime}{ }_{3}-b^{4} \mathrm{~F}^{\prime}{ }_{4} \\
y^{4} \mathrm{P}_{3}-e^{4} \mathrm{P}_{3}^{\prime} \\
z^{4} \mathrm{D}_{4}^{\prime}-b^{4} \mathrm{~F}_{5}^{\prime} \\
z^{2} \mathrm{~F}_{3}-c^{2} \mathrm{D}_{2}\end{array}$ & & & & \\
\hline
\end{tabular}


TABLE 6.-Classified lines of $Y t I$-Continued

\begin{tabular}{|c|c|c|c|c|c|c|c|c|}
\hline \multirow{2}{*}{$\lambda$} & \multirow{2}{*}{$\begin{array}{c}\text { Intensity } \\
\mathbf{M}\end{array}$} & \multirow{2}{*}{$\begin{array}{c}\text { Intensity } \\
\text { Class K }\end{array}$} & \multirow{2}{*}{ - $\quad \nu$} & \multirow{2}{*}{ Terms } & \multicolumn{2}{|c|}{ Zeeman effect } & \multicolumn{2}{|c|}{ Solar inteusity } \\
\hline & & & & & Observed & Calculated & Disk & Spot \\
\hline $\begin{array}{l}7,887.51 \\
7,999.33 \\
8,025.60 \\
8,134.9 \\
8,211.71\end{array}$ & $\begin{array}{l}2 \\
3 \\
3 \\
3 \\
4\end{array}$ & & $\begin{array}{r}12,675.0 \\
497.6 \\
456.7 \\
289.3 \\
174.4\end{array}$ & $\begin{array}{l}z^{4} \mathrm{D}^{\prime}{ }_{2}-b^{4} \mathrm{~F}^{\prime}{ }^{\prime}{ }^{4} \mathrm{D}^{\prime}{ }_{-1}-b^{4} \mathrm{~F}^{\prime}{ }_{4} \\
y^{4} \mathrm{~F}_{4}-e^{4} \mathrm{~F}^{\prime}{ }_{4} \\
y^{2} \mathrm{D}_{2}^{\prime}-d^{2} \mathrm{D}_{2} \\
y^{2} \mathrm{~F}_{4}-\left(1_{3}\right)\end{array}$ & & & & \\
\hline $\begin{array}{l}8,231.23 \\
8,329.61 \\
8,344.43 \\
8,365.64 \\
8,450.36\end{array}$ & $\begin{array}{l}2 \\
5 \\
10 \\
4 \\
8\end{array}$ & & $\begin{array}{r}145.5 \\
12,002.1 \\
11,980.8 \\
950.4 \\
830.6\end{array}$ & $\begin{array}{l}b^{2} \mathrm{D}_{2}-x^{2} \mathrm{P}_{2} \\
y^{4} \mathrm{~F}_{5}-d^{4} \mathrm{~F}^{\prime}{ }_{5} \\
b^{2} \mathrm{D}_{3}-x^{2} \mathrm{P}_{2} \\
y^{2} \mathrm{P}_{2}-d^{2} \mathrm{D}_{3} \\
b^{2} \mathrm{D}_{2}-x^{2} \mathrm{P}_{1}\end{array}$ & & & & \\
\hline $\begin{array}{l}8,475.64 \\
8,52.94 \\
8,556.04 \\
8,575.77 \\
8,627.9\end{array}$ & $\begin{array}{l}3 \\
4 \\
2 \mathrm{~d} ? \\
2 \\
1\end{array}$ & & $\begin{array}{l}795.3 \\
721.6 \\
684.4 \\
657.6 \\
587.2\end{array}$ & $\begin{array}{l}y^{4} \mathrm{~F}_{4}-d^{4} \mathrm{~F}^{\prime}{ }_{4} \\
y^{2} \mathrm{P}_{1}-d^{2} \mathrm{D}_{2} \\
y^{2} \mathrm{D}^{\prime} \mathrm{D}_{3}-\mathrm{d}^{2} \mathrm{D}_{3} \\
y^{4} \mathrm{~F}_{3}-d^{4} \mathrm{~F}^{\prime} \\
y^{4} \mathrm{~F}_{2}-d^{4} \mathrm{~F}^{\prime}{ }_{2}\end{array}$ & & 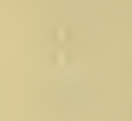 & & \\
\hline $\begin{array}{l}8,800.62 \\
9,231.58 \\
9,494.81\end{array}$ & $\begin{array}{l}10 \\
8 \\
2\end{array}$ & & $\begin{array}{l}11,359.7 \\
10,829.4 \\
10,529.2\end{array}$ & $\begin{array}{l}a^{2} \mathrm{D}_{2}-z^{2} \mathrm{P}_{2} \\
a^{2} \mathrm{D}_{3}-z^{2} \mathrm{P}_{2} \\
a^{2} \mathrm{D}_{2}-z^{2} \mathrm{P}_{1}\end{array}$ & & & & \\
\hline
\end{tabular}

TABLE 7.-Unclassified lines of Yt I

\begin{tabular}{|c|c|c|c|c|c|c|c|}
\hline$\lambda$ & Intensity & Temp. class & $\nu$ & $\lambda$ & Intensity & Temp. class & $\nu$ \\
\hline $\begin{array}{r}2,826.29 \\
2,835.78 \\
3,044.84 \\
47.11 \\
47.41\end{array}$ & 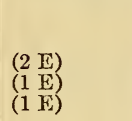 & $\begin{array}{l}1 \text { IV A } \\
1 \text { IV } \\
5 \text { III } \\
3 \text { III } \\
3 \text { III }\end{array}$ & $\begin{array}{r}35,371.7 \\
35,253.3 \\
32,832.9 \\
808.5 \\
805.2\end{array}$ & $\begin{array}{r}4,300.34 \\
15.48 \\
22.30 \\
29.89 \\
4,387.74\end{array}$ & $\begin{array}{l}2 \\
2 \\
2 \\
1 \\
4\end{array}$ & $\begin{array}{l}2 \text { III } \\
2 \text { III } \\
2 \text { III } \\
1 \text { III } \\
8 \text { III }\end{array}$ & $\begin{array}{r}23,247.4 \\
165.9 \\
129.3 \\
23,088.8 \\
22,784.4\end{array}$ \\
\hline $\begin{array}{r}56.33 \\
59.50 \\
72.32 \\
76.49 \\
3,091.70\end{array}$ & 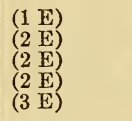 & $\begin{array}{l}2 ? \mathrm{III} ? \\
4 \mathrm{III} \\
5 \mathrm{III} \\
5 \mathrm{III} \\
15 \mathrm{III}\end{array}$ & $\begin{array}{l}709.5 \\
675.6 \\
539.3 \\
495.2 \\
335.3\end{array}$ & $\begin{array}{r}4,465.27 \\
72.78 \\
4,473.88 \\
4,564.37 \\
4,590.77\end{array}$ & $\begin{array}{l}3 \\
2 \\
5 \\
2 \\
(3 \mathrm{E})\end{array}$ & $\begin{array}{l}1 \mathrm{IV} \\
2 \mathrm{n} \mathrm{IV} \\
7 \mathrm{III} \\
4 \mathrm{III} \\
1 \mathrm{~V}\end{array}$ & $\begin{array}{r}388.8 \\
351.2 \\
22,345.7 \\
21,902.7 \\
21,776.8\end{array}$ \\
\hline $\begin{array}{r}3,103.69 \\
14.27 \\
3,171.69 \\
3,252.28 \\
75.56\end{array}$ & 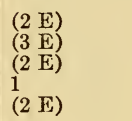 & $\begin{array}{l}4 \mathrm{IV} \\
2 \mathrm{III} \\
4 \mathrm{III} \mathrm{A} \\
8 \mathrm{III} \\
2 \mathrm{IV}\end{array}$ & $\begin{array}{r}210.4 \\
32,101.0 \\
31,519.8 \\
30,738.8 \\
520.4\end{array}$ & $\begin{array}{l}4,658.88 \\
4,670.82 \\
4,863.11 \\
4,912.05 \\
4,950.64\end{array}$ & $\begin{array}{l}3 \\
2 \\
(2 \mathrm{E}) \\
1 \\
3\end{array}$ & $\begin{array}{l}6 \text { III } \\
4 \text { III A } \\
1 \text { III } \\
\text { tr III A ? } \\
2 \text { III }\end{array}$ & $\begin{array}{r}458.4 \\
21,403.5 \\
20,557.2 \\
352.4 \\
20,193.8\end{array}$ \\
\hline $\begin{array}{r}78.43 \\
3,287.21 \\
3,335.20 \\
44.53 \\
82.83\end{array}$ & $\begin{array}{l}(2 \mathrm{E}) \\
(3 \mathrm{E}) \\
(2 \mathrm{E}) \\
(2 \mathrm{E}) \\
(1 / 2 \mathrm{E})\end{array}$ & $\begin{array}{l}5 \mathrm{III} \mathrm{A} \\
4 \mathrm{III} \\
8 \mathrm{n} \mathrm{V} \\
4 \mathrm{n} \mathrm{V} \\
5 \mathrm{~V}\end{array}$ & $\begin{array}{r}493.6 \\
30,412.2 \\
29,974.6 \\
891.0 \\
552.6\end{array}$ & $\begin{array}{l}5,072.19 \\
5,686.62 \\
5,713.81 \\
6,251.05 \\
6,338.14\end{array}$ & $\begin{array}{l}3 \\
(1 \mathrm{E}) \\
1 \mathrm{~h} \\
3 \\
3\end{array}$ & $\begin{array}{l}5 \mathrm{IV} \\
1 \mathrm{IV} ? \\
3 \mathrm{n} \mathrm{IV} \\
5 \mathrm{I}\end{array}$ & $\begin{array}{r}19,709.9 \\
17,580.3 \\
17,496.6 \\
15,992.9 \\
773.1\end{array}$ \\
\hline $\begin{array}{r}83.06 \\
3,394.97 \\
3.414 .5 \\
24.16 \\
31.67\end{array}$ & $\begin{array}{l}(1 \mathrm{E}) \\
(2 \mathrm{E} \& \mathrm{H}) \\
(2 \mathrm{E} \& \mathrm{H}) \\
(2 \mathrm{E} \& \mathrm{H})\end{array}$ & $\begin{array}{l}7 \mathrm{~V} \\
4 \mathrm{III} \\
8 \mathrm{n} \text { III } \\
7 \text { III } \\
5 \text { III }\end{array}$ & $\begin{array}{l}550.6 \\
446.9 \\
278.5 \\
195.9 \\
132.0\end{array}$ & $\begin{array}{l}6,462.59 \\
7,536.73 \\
7,698.00 \\
8,165.5 \\
8,258.5\end{array}$ & $\begin{array}{l}2 \\
3 \\
4 \\
2 \\
2\end{array}$ & $8 \mathrm{III}$ & $\begin{array}{r}15,469.4 \\
13,264.2 \\
12,986.8 \\
243.2 \\
105.4\end{array}$ \\
\hline $\begin{array}{r}33.79 \\
37.95 \\
71.7 \\
3,473.18 \\
3,500.60\end{array}$ & $\begin{array}{l}(2 \mathrm{E} \& \mathrm{H}) \\
1 \\
1 \\
(2 \mathrm{E} \& \mathrm{H}) \\
1\end{array}$ & $\begin{array}{l}6 \mathrm{n} \mathrm{IV} \\
8 \mathrm{nIV} \\
10 \mathrm{nV} ? \\
5 \mathrm{III} \\
7 \mathrm{III}\end{array}$ & $\begin{array}{r}114.0 \\
29,078.8 \\
28,796.1 \\
783.8 \\
558.4\end{array}$ & $\begin{array}{l}8,297.07 \\
8,326.40 \\
8,330.92 \\
8,443.28 \\
8,552.42\end{array}$ & $\begin{array}{l}2 \\
1 \\
1 \\
1 \\
1\end{array}$ & & $\begin{array}{r}049.2 \\
006.7 \\
12,000.2 \\
11,840.5 \\
689.4\end{array}$ \\
\hline $\begin{array}{r}06.47 \\
3,587.75 \\
3,904.59 \\
3,955.08 \\
4,224.25\end{array}$ & $\begin{array}{l}(2 \mathrm{E} \& \mathrm{H}) \\
5 \\
2 \\
3 \\
3\end{array}$ & $\begin{array}{l}6 \text { III } \\
20 \text { III } \\
5 \text { III } \\
5 \text { III } \\
4 \text { III }\end{array}$ & $\begin{array}{l}28,510.6 \\
27,864.7 \\
25,603.7 \\
25,276.8 \\
23,666.2\end{array}$ & $\begin{array}{l}8,658.4 \\
8,759.24 \\
8,831.2 \\
9,392.7\end{array}$ & $\begin{array}{l}1 \\
1 \\
1 \\
1\end{array}$ & & $\begin{array}{r}546.3 \\
413.4 \\
11,320.4 \\
10,643.6\end{array}$ \\
\hline
\end{tabular}




\section{THEORETICAL INTERPRETATION OF THE SPARK SPECTRA AND COMPARISON WITH SCANDIUM}

In our paper on scandium spectra we gave a summary of the theoretical advances which correlate the observed spectral terms with the electron configurations in the atoms producing them. There is no necessity for repeating this, especially since the subject has since been discussed in detail in Hund's notable monograph.

A. Yt III. The relatively simple spectrum of doubly ionized yttrium has already been discussed; there is only one valency electron, and its various orbits are responsible for the doublet terms as in the arc spectrum of an alkali. Analysis of the Yt III spectrum shows that the $4 d$ orbit is most firmly bound, $5 s, 5 p$, and $4 f$ being next in order.

A comparison of Yt III and Sc III ${ }^{20}$ is given in Table 8.

TABLE 8.-Comparison of Yt III and Sc III

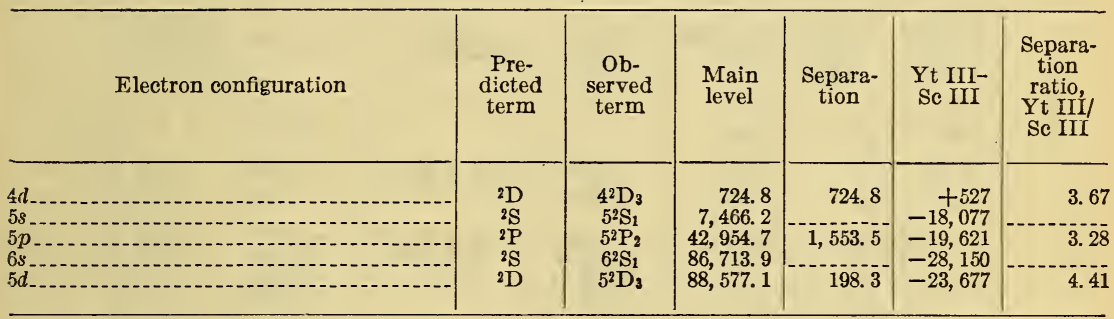

The corresponding terms in scandium have total quantum numbers smaller by one unit. The difference of binding for the lowest $d$ and $s$ electrons is less for Yt III than for Sc III. The higher s-electron is more firmly bound than the $d$ in both cases.

B. Yt II. The singly ionized yttrium atom has two valency electrons, and the spectral terms (atomic energy states) corresponding to their various configurations are readily obtained by adding an electron to the lower electron configurations of $\mathrm{Yt}^{++}$. The addition of a $5 \mathrm{~s}$ electron to the normal $4 d$ state should give the terms ${ }^{3} \mathrm{D}$ and ${ }^{1} \mathrm{D}$; that of a $4 d$ electron to the same state produces ${ }^{3} \mathrm{~F}^{\prime},{ }^{3} \mathrm{P}^{\prime},{ }^{1} \mathrm{G},{ }^{1} \mathrm{D},{ }^{1} \mathrm{~S}$, while adding a $5 s$ electron to one already present gives ${ }^{1} \mathrm{~S}$ alone. These terms account completely for the observed terms of the low set (leaving one ${ }^{1} \mathrm{~S}$ term to be discovered). Since their configurations contain no $p$ or $f$ electrons, these terms are all "even" and do not combine among themselves. The addition of a $5 p$ electron to the $4 d$ and $5 s$ states of $\mathrm{Yt}^{++}$gives the middle set of terms, exactly as observed, which are "odd" and combine with the lowest terms and also with the higher "even" terms arising from several configurations.

20 Russell and Lang, Astrophys J., 66, p. 20; 1927. 
Table 9 gives the predicted terms corresponding to each configuration and the observed terms with which they have been identified, and also a comparison with Sc II similar to that made for Sc III. The identification of the lower and middle levels is obvious - the wide separation of $z^{3} \mathrm{P}$ showing that it belongs to the $s p$ configuration. Among the higher terms the pentads coming from $4 d .5 d$ are recognizable by the strength of their combinations and the relative intensities of the different multiplets. The terms $b^{3} \mathrm{D}, c^{1} \mathrm{D}$, and $c^{1} \mathrm{~S}$ are determined by the series relations discussed below; $a^{3} \mathrm{~S}$ and $d^{3} \mathrm{D}$ are placed because of the strength of their combinations with $z^{3} \mathrm{P}(s p)$ and also by the narrow separation of the latter which belongs to a series converging toward the single limit $5^{2} \mathrm{~S}$ of $\mathrm{Yt}$ III. The wide separation of $b^{3} \mathrm{P}^{\prime}$, as well as its high level, assigns it to $(5 p)^{2}$, and the term $f^{1} \mathrm{D}$ is too high to belong to any but this configuration. The assignment of $e^{1} \mathrm{D}$ is uncertain, but it fits in well where it is placed.

TABLE 9.-Predicted and observed terms in the $Y t I I$ spectrum

\begin{tabular}{|c|c|c|c|c|c|c|}
\hline $\begin{array}{l}\text { Electron } \\
\text { configu- } \\
\text { ration }\end{array}$ & $\begin{array}{l}\text { Pre- } \\
\text { dicted } \\
\text { term }\end{array}$ & $\begin{array}{c}\text { Observed } \\
\text { term }\end{array}$ & $\begin{array}{l}\text { Main } \\
\text { level }\end{array}$ & $\begin{array}{c}\text { Level } \\
\text { separations }\end{array}$ & Yt II-Sc II & $\begin{array}{l}\text { Ratio of } \\
\text { separation } \\
\text { Yt II/Sc II }\end{array}$ \\
\hline$(5 s)^{2}$ & ${ }^{1} \mathrm{~S}_{0}$ & $a^{1} \mathrm{~S}_{0}$ & 0.0 & & $-11,736$ & \\
\hline $5 s \cdot 4 d$ & ${ }^{3 \mathrm{D}}$ & $\begin{array}{l}a^{3} \mathrm{D}_{3} \\
a^{1} \mathrm{D}_{2}\end{array}$ & $\begin{array}{l}1,449.9 \\
3,296.3\end{array}$ & $404.8 ; 204.9$ & $\begin{array}{r}+1,272 \\
+755\end{array}$ & 3.42 \\
\hline$(4 d)^{2}$ & $\begin{array}{l}{ }^{3} \mathrm{~F}^{\prime} \\
{ }^{3} \mathrm{P}^{\prime} \\
{ }^{1} \mathrm{G} \\
{ }^{1} \mathrm{D} \\
{ }^{1} \mathrm{~S}\end{array}$ & $\begin{array}{l}a^{3} \mathrm{~F}^{\prime}{ }_{4} \\
a^{3} \mathrm{P}^{\prime}{ }_{2} \\
a^{1} \mathrm{G}_{4} \\
b^{1} \mathrm{D}_{2} \\
?\end{array}$ & $\begin{array}{r}8,743.4 \\
14,098.2 \\
15,683.0 \\
14,832.9\end{array}$ & $\begin{array}{r}415.4 ; 324.9 \\
79.8 ; 134.9\end{array}$ & $\begin{array}{l}+3,756 \\
+1,944 \\
+1,422 \\
+3,888\end{array}$ & $\begin{array}{l}4.00 \\
2.66\end{array}$ \\
\hline $5 s \cdot 5 p$ & ${ }_{1 \mathrm{P}}^{3 \mathrm{P}}$ & $\begin{array}{l}z^{3} \mathrm{P}_{2} \\
y^{1} \mathrm{P}_{1}\end{array}$ & $\begin{array}{l}24,647.2 \\
44,568.2\end{array}$ & $870.9 ; 331.2$ & $\begin{array}{l}-14,698 \\
-11,148\end{array}$ & 3. 50 \\
\hline $4 d \cdot 5 p$ & $\begin{array}{l}{ }^{3} \mathrm{P} \\
{ }^{3} \mathrm{D}^{\prime} \\
{ }^{3} \mathrm{~F} \\
{ }^{1} \mathrm{P} \\
{ }^{1} \mathrm{D}^{\prime} \\
{ }^{1} \mathrm{~F}\end{array}$ & $\begin{array}{l}y^{3} \mathrm{P}_{2} \\
z^{3} \mathrm{D}_{3}^{\prime} \\
z^{3} \mathrm{~F}_{4} \\
2^{1} \mathrm{P}_{1} \\
z^{1} \mathrm{D}_{2}^{\prime}{ }_{2} \\
z^{1} \mathrm{~F}_{3}\end{array}$ & $\begin{array}{l}32,283.6 \\
29,214.1 \\
28,394.3 \\
27,516.7 \\
26,147.2 \\
33,336.7\end{array}$ & $\begin{array}{l}\text { 159. } 5 ; 75.3 \\
484.0 ; 134.8 \\
862.1 ; 305.3\end{array}$ & $\begin{array}{r}+2,460 \\
+1,053 \\
+553 \\
-3,299 \\
+66 \\
+987\end{array}$ & $\begin{array}{l}2.79 \\
2.54 \\
2.94\end{array}$ \\
\hline $58 \cdot 68$ & $\begin{array}{l}{ }^{3} \mathrm{~S} \\
{ }^{1} \mathrm{~S}\end{array}$ & $\begin{array}{l}a^{3} \mathrm{~S}_{1} \\
c^{1} \mathrm{~S}_{0}\end{array}$ & $\begin{array}{l}58,261.7 \\
61,367.3\end{array}$ & 1) & & \\
\hline $4 d \cdot 6 s$ & ${ }^{3} \mathrm{D}$ & $\begin{array}{l}b^{3} \mathrm{D}_{3} \\
c^{1} \mathrm{D}_{2}\end{array}$ & $\begin{array}{l}55,645.4 \\
55,724.9\end{array}$ & $613.4 ; 76.4$ & $\begin{array}{l}-2,098 \\
-2,527\end{array}$ & 3.59 \\
\hline $5 s \cdot 5 d$ & $\begin{array}{l}{ }^{3} \mathrm{D} \\
{ }^{D} \mathrm{D}\end{array}$ & $\begin{array}{l}d^{3} \mathrm{D}_{3} \\
e^{1} \mathrm{D}_{2}\end{array}$ & $\begin{array}{l}65,275.0 \\
62,495.2\end{array}$ & $86.3 ; 57.2$ & & \\
\hline $4 d \cdot 5 d$ & $\begin{array}{l}{ }^{3} \mathrm{G} \\
{ }^{3} \mathrm{~F}^{\prime} \\
{ }^{3} \mathrm{D} \\
{ }^{3} \mathrm{P} \\
{ }^{3} \mathrm{~S} \\
{ }^{1} \mathrm{G} \\
{ }^{1} \mathrm{~F}^{\prime} \\
{ }^{1} \mathrm{D} \\
{ }^{1} \mathrm{P}^{\prime} \\
{ }^{1} \mathrm{~S}_{0}\end{array}$ & $\begin{array}{l}a^{3} \mathrm{G}_{3} \\
b^{3} \mathrm{~F}^{\prime}{ }_{4} \\
c^{3} \mathrm{D}_{3} \\
c^{3} \mathrm{P}^{\prime}{ }_{2} \\
b^{3} \mathrm{~S}_{1} \\
b^{1} \mathrm{G}_{4} \\
a^{1} \mathrm{~F}^{\prime}{ }_{3} \\
d^{1} \mathrm{D}_{2} \\
a^{1} \mathrm{P}_{1}^{\prime} \\
b^{1} \mathrm{~S}_{0}\end{array}$ & $\begin{array}{l}59,900.4 \\
61,934.1 \\
59,327.1 \\
64,597.1 \\
61,200.2 \\
63,350.0 \\
58,533.0 \\
60,535.1 \\
59,715.4 \\
59,615.8 ?\end{array}$ & $\begin{array}{l}428.6 ; 292.9 \\
283.6 ; 313.8 \\
380.5 ; 226.9 \\
334.4 ; 159.8\end{array}$ & $\begin{array}{r}-557 \\
-1,593 \\
-674 \\
-108 \\
+129 \\
-1,886 \\
-995 \\
-3,831 \\
-685 \\
-5,328 ?\end{array}$ & $\begin{array}{l}3.81 \\
3.88 \\
4.79 \\
5.50\end{array}$ \\
\hline$(5 p)^{2}$ & $\begin{array}{l}{ }^{3} \mathrm{P}^{\prime} \\
f_{1 \mathrm{~S}}^{1} \mathrm{D}\end{array}$ & $\begin{array}{l}b^{3} \mathrm{P}^{\prime}{ }_{2} \\
f^{1} \mathrm{D}_{2} \\
?\end{array}$ & $\begin{array}{l}59,669.6 \\
70,223.2\end{array}$ & $522.4 ; 371.5$ & $-16,919$ & 2. 58 \\
\hline
\end{tabular}

The comparison of the levels and separations with those in Sc II is of much interest. The separations are always much wider in Yt II, the mean of 13 ratios being 3.54 as against 3.78 for Yt III 
compared with Sc III. In comparing the levels it should be remembered that they are measured from different origins ${ }^{1} \mathrm{~S}_{0}$ for $\mathrm{Yt}$ II and ${ }^{3} \mathrm{D}_{1}$ for Sc II. In discussing them it will be well to use similar origins, which may be done by subtracting 1,272 from the tabular values, so that the ${ }^{3} \mathrm{D}_{3}$ levels agree. The mean differences in level for the various electron configurations are then found to be:

\begin{tabular}{|c|c|c|c|c|c|}
\hline $\begin{array}{l}\text { Configu- } \\
\text { ration }\end{array}$ & $\begin{array}{l}\text { Difference } \\
\text { Yt II-Sc II }\end{array}$ & $\begin{array}{l}\text { Number } \\
\text { of cases }\end{array}$ & $\begin{array}{l}\text { Configu- } \\
\text { ration }\end{array}$ & $\begin{array}{l}\text { Difference } \\
\text { Yt II-Sc II }\end{array}$ & $\begin{array}{l}\text { Number } \\
\text { of cases }\end{array}$ \\
\hline $\begin{array}{l}s^{2} \\
s p \\
p^{2} \\
d s\end{array}$ & $\begin{array}{r}-13,008 \\
-14,195 \\
-18,191 \\
-259\end{array}$ & $\begin{array}{l}1 \\
2 \\
1 \\
2\end{array}$ & $\begin{array}{r}d^{2} \\
d p \\
d \cdot s \\
d \cdot d\end{array}$ & $\begin{array}{r}-1,480 \\
-969 \\
-3,584 \\
-2,411\end{array}$ & $\begin{array}{r}4 \\
6 \\
12 \\
29\end{array}$ \\
\hline
\end{tabular}

1 The symbols $d \cdot s$ and $d \cdot d$ denote configurations with one electron excited to a higher quantum state. 2 The term $b^{1 S}$ in $\mathrm{Yt}$ II has been omitted from the last mean. It gives such a discordant difference as to make its reality very doubtful.

For the configurations which contain a $d$-electron the relative levels are much the same in $\mathrm{Sc}^{+}$and $\mathrm{Yt}^{+}$except that those involving an excited electron are a little lower in the latter because of the greater ease of ionization. Those configurations which do not contain a $d$-electron are very much lower in $\mathrm{Yt}^{+}$. This is obviously a consequence of the fact that the $s$ and $p$ states of $\mathrm{Yt}^{+++}$, from which these configurations are derived, are lower compared with the $d$ state than in $\mathrm{Sc}^{++}$. The differences in the two first spark spectra, which involves a change in the nature of the normal terms or states of the ions, could, therefore, have been predicted from the second spark spectra.

\section{A NOTE ON Sc II}

The considerations just developed have led to a change in one of the conclusions expressed in our previous paper regarding the interpretation of the terms of Sc II. The term $a^{1} \mathrm{~S}_{0}$ at the level 11,736.35 was then attributed to the configuration $(3 d)^{2}$, while the similar term arising from $(4 s)^{2}$ was not found. The ${ }^{1} \mathrm{P}$ term from $4 s 4 p$ was also missing. Comparison with yttrium suggests that the observed ${ }^{1} \mathrm{~S}$ term belongs to the $s^{2}$ configuration and is homologous with the lowest energy level of Yt II. If so, it should give a strong combination with the term ${ }^{1} \mathrm{P}(s p)$. This term has now been identified at the level 55,715.52; it combines with $a^{1} \mathrm{~S}_{0}$ and $b^{1} \mathrm{D}$ to give the one remaining strong spark line $(2273 \mathrm{~A})$ and a faint one recorded by Exner and Haschek, as follows:

\begin{tabular}{|c|c|c|c|c|c|}
\hline \multicolumn{2}{|c|}{ Terms } & $\nu$ calculated & $\nu$ observed & $\lambda$ observed & Intensity \\
\cline { 1 - 2 }$b^{1} \mathrm{P}_{2}$ & $55,715.52$ & & & & \\
$a^{1} \mathrm{~S}_{0}$ & $11,736.35$ & $43,979.17$ & $43,979.17$ & $2,273.10$ & 3 \\
$b^{1} \mathrm{D}_{2}$ & $10,944.51$ & $44,771.0$ & $44,769.7$ & $2,232.96$ & 1 \\
\hline
\end{tabular}


For the stronger line Exner and Haschek's wave length is $0.08 \mathrm{~A}$ greater than Meggers's. If a corresponding correction is applied to the fainter line, the agreement with the computed position is exact. These terms have been used in calculating the differences entered in the appropriate places in Table 9.

It is now clear that the Sc II and Yt II spectra are exactly analogous; every line in the first has its correlative in the second. The only difference in the observed spectra is found in the apparent absence in Sc II of the terms coming from the configuration $s \cdot s$ and $s \cdot d$. These terms are already high in Yt II, and should be fully 12,000 frequency units higher in Sc II; their combinations will be faint and far in the ultra-violet.

\section{INTERPRETATION OF THE TERMS OF Yt I}

The presence of three electrons in neutral yttrium atoms greatly increases the number of possible terms and thus accounts for the greater complexity of this spectrum. The possible electron configurations and corresponding terms are tabulated in Table 10, where a summary of the observed terms, their main levels and level separations, are also given. In cases where the assignment is uncertain the observed term is preceded by a question mark. The comparison with the levels and separations in Sc I is made as before. A few terms which were not found in the Sc I spectrum have been identified in the Yt I spectrum, and vice versa, but in general there is a remarkably close correspondence of the terms of the two spectra.

The interpretation of the low even levels is simple. The $d s^{2}$ configuration gives the normal state, $a^{2} \mathrm{D}_{1}$, while $d^{2} s$ gives ${ }^{4} \mathrm{~F}^{\prime},{ }^{4} \mathrm{P}^{\prime}$, ${ }^{2} \mathrm{D},{ }^{2} \mathrm{~F}^{\prime},{ }^{2} \mathrm{P}^{\prime}$ and a higher ${ }^{2} \mathrm{D}$. The ${ }^{2} \mathrm{~S}$ term which should be produced by this configuration has not been identified. The same is true for Sc I.

The middle terms are also easy to classify. Most of them should be arranged in triads, combining strongly with one of the low terms, and all these triads are clearly present, except for the ${ }^{2} \mathrm{P}$ term combining with $a^{2} \mathrm{P}^{\prime}$. The configuration $s^{2} p$ should give an isolated ${ }^{2} \mathrm{P}$ term, which we may expect to be low (since no $d$ electron is involved) and of wide separation. This is evidently $z^{2} \mathrm{P}$. All the odd terms are now accounted for. 
TABLE 10.-Predicted and observed terms in the Yt I spectrum

\begin{tabular}{|c|c|c|c|c|c|c|}
\hline $\begin{array}{l}\text { Electron } \\
\text { configuration }\end{array}$ & $\begin{array}{l}\text { Predicted } \\
\text { terms }\end{array}$ & $\begin{array}{c}\text { Observed } \\
\text { terms }\end{array}$ & Main level & Level separations & Yt I-Sc I & $\begin{array}{l}\text { Ratio of } \\
\text { separations } \\
\text { Yt I/Sc I }\end{array}$ \\
\hline$(5 s)^{2} .4 d$ & ${ }^{2} \mathrm{D}$ & $a^{2} \mathrm{D}_{3}$ & 530.5 & 530.5 & +362 & 3.16 \\
\hline \multirow[t]{2}{*}{$5 s(4 d)^{2}$} & $\begin{array}{l}{ }^{4} \mathrm{~F}^{\prime} \\
{ }^{4} \mathrm{P}\end{array}$ & $\begin{array}{l}a^{4} F^{\prime}{ }^{5} \\
a^{4} \mathrm{P}^{\prime}{ }_{3}\end{array}$ & $\begin{array}{l}11,532.1 \\
15,476.7\end{array}$ & $\begin{array}{l}254.1,199.4,141.2 \\
147.7,107.3\end{array}$ & -1.45 & 3.78 \\
\hline & $\begin{array}{l}{ }^{2} \mathrm{G} \\
{ }^{2} \mathrm{~F}^{\prime} \\
{ }^{2} \mathrm{D} \\
{ }^{2} \mathrm{P}^{\prime} \\
{ }^{\prime} \mathrm{S}\end{array}$ & $\begin{array}{l}a^{2} \mathrm{C}_{5} \\
a^{2} \mathrm{~F}^{\prime}{ }_{4} \\
b^{2} \mathrm{D}_{3} \\
a^{2} \mathrm{P}^{\prime}\end{array}$ & $\begin{array}{l}18,499.2 \\
15,864.4 \\
16,158.8 \\
19,406.1\end{array}$ & $\begin{array}{l}-13.2 \\
537.6 \\
164.8 \\
168.4\end{array}$ & $\begin{array}{r}-1,738 \\
+822 \\
-854\end{array}$ & $\begin{array}{r}4.68 \\
2.75 \\
(-13.3) \\
(2.10 ?)\end{array}$ \\
\hline \multirow[t]{2}{*}{$(4 d)^{3}$} & $\begin{array}{l}{ }^{4} \mathrm{~F}^{\prime} \\
{ }^{4} \mathrm{P}^{\prime}\end{array}$ & $\begin{array}{l}b^{4} \mathrm{~F}^{\prime}{ }^{5} \\
b^{4} \mathrm{P}^{\prime}{ }_{3}\end{array}$ & $\begin{array}{l}29,842.6 \\
32,366.3\end{array}$ & $\begin{array}{l}229.0,193.3,148.3 \\
275.3,113.5\end{array}$ & $\begin{array}{l}-4,064 \\
-4,206\end{array}$ & $\begin{array}{l}4.00 \\
4.80\end{array}$ \\
\hline & $\begin{array}{l}{ }^{2} \mathrm{H}^{\prime} \\
{ }^{2} \mathrm{G} \\
{ }^{2} \mathrm{~F}^{\prime} \\
{ }^{2} \mathrm{D} \\
{ }^{2} \mathrm{P}^{\prime}\end{array}$ & & & - & & \\
\hline$(5 s)^{2} .5 p$ & ${ }^{2} \mathrm{P}$ & $z^{2} \mathrm{P}_{2}$ & $11,359.7$ & 830.5 & $-7,495$ & 5.76 \\
\hline \multirow[t]{3}{*}{ ss.4d.5p. } & $\begin{array}{l}{ }^{4 F} \\
{ }^{4} \mathrm{D} \\
{ }^{4} \mathrm{P}\end{array}$ & $\begin{array}{l}z^{4} \mathrm{~F}_{5} \\
z^{4} D^{\prime{ }^{\prime}} \\
z^{4} \mathrm{P}_{3}\end{array}$ & $\begin{array}{l}16,234.5 \\
17,116.3 \\
19,148.0\end{array}$ & $\begin{array}{l}522.1,466.6,296.8 \\
299.7,219.3,161.5 \\
120.5,51.2\end{array}$ & $\begin{array}{l}+208 \\
+906 \\
+577\end{array}$ & $\begin{array}{l}3.63 \\
\begin{array}{l}3.39 \\
2.55\end{array}\end{array}$ \\
\hline & $\begin{array}{l}2 \mathrm{~F} \\
{ }_{2 \mathrm{P}}^{\prime}\end{array}$ & $\begin{array}{l}z^{2} \mathrm{~F}_{4} \\
z^{2} D^{\prime}{ }^{3} \\
z^{\prime 2} \mathrm{P}_{2}\end{array}$ & $\begin{array}{l}21,915.4 \\
16,068.0 \\
24,480.6\end{array}$ & $\begin{array}{l}386.8 \\
-80.1 \\
-218.2\end{array}$ & $\begin{array}{r}+830 \\
+43 \\
-176\end{array}$ & $\begin{array}{r}(7.28) \\
(1.07) \\
(\infty)\end{array}$ \\
\hline & 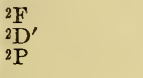 & $\begin{array}{l}y^{2} \mathrm{~F}_{4} \\
y^{2}{ }^{\prime}{ }^{\prime} \\
x^{2} \mathrm{P}_{2}\end{array}$ & $\begin{array}{l}24,899.5 \\
24,746.6 \\
28,139.6\end{array}$ & $\begin{array}{l}380.7 \\
615.4 \\
315.1\end{array}$ & $\begin{array}{r}+2,315 \\
-268 \\
-2,567\end{array}$ & $\begin{array}{l}2.72 \\
4.16 \\
2.36\end{array}$ \\
\hline \multirow[t]{7}{*}{$(1 d)^{2} .5 p$} & $\begin{array}{l}{ }^{4} \mathrm{G}^{\prime} \\
4^{4} \mathrm{~F} \\
{ }^{4} \mathrm{D}^{\prime}\end{array}$ & $\begin{array}{l}z^{4} \mathrm{G}^{\prime} 6 \\
y^{4} F_{5}^{5} \\
y^{4} \mathrm{D}^{\prime} 4\end{array}$ & $\begin{array}{l}29,820.4 \\
32,188.1 \\
33,614.5\end{array}$ & $\begin{array}{l}456.2,375.3,294.9 \\
278.9,229.2,171.6 \\
256.9,92.3,40.9\end{array}$ & $\begin{array}{l}+517 \\
+837 \\
+863\end{array}$ & $\begin{array}{l}\text { 4. } 00 \\
\text { 4. } 37 \\
\text { 3. } 50\end{array}$ \\
\hline & $\begin{array}{l}{ }^{4} \mathrm{D}^{\prime} \\
{ }_{4}^{4} \mathrm{~S}^{\prime}\end{array}$ & $\begin{array}{l}x^{4} \mathrm{D}_{4}^{\prime} \\
y^{4}{ }_{3} \\
z^{2} \mathrm{~S}_{2}^{\prime}\end{array}$ & $\begin{array}{l}36,361.3 \\
37,476.0 \\
36,750.8\end{array}$ & $\begin{array}{l}300.3,-74.7,319.0 \\
327.5,109.0\end{array}$ & $\begin{array}{l}(?) \\
(?) \\
(?)\end{array}$ & (?) \\
\hline & $\begin{array}{l}{ }^{2} \mathrm{H} \\
{ }^{2} \mathrm{G} \\
{ }^{\prime} \mathrm{G} \\
{ }^{\mathrm{F}}\end{array}$ & $\begin{array}{l}z^{2} \mathrm{H}_{8} \\
y^{2} \mathrm{G}^{\prime} \\
v^{2} \mathrm{~F}_{4}\end{array}$ & $\begin{array}{l}37,967.2 \\
38,590.7 \\
42,994.8\end{array}$ & $\begin{array}{l}379.0 \\
117.7 \\
136.9\end{array}$ & $\begin{array}{r}-1,282 \\
-827 \\
+3,106\end{array}$ & $\begin{array}{r}3.95 \\
3.83 \\
(17.3)\end{array}$ \\
\hline & $\begin{array}{l}{ }^{2} \mathrm{G}^{\prime} \\
{ }^{\mathrm{F}} \\
{ }^{2} \mathrm{D}^{\prime}\end{array}$ & $\begin{array}{l}z^{2} \mathrm{G}^{\prime} 5 \\
x^{2 F_{4}^{4}} \\
x^{2} \mathrm{D}^{\prime}{ }_{3}\end{array}$ & $\begin{array}{l}33,788.8 \\
34,029.8 \\
34,247.7\end{array}$ & $\begin{array}{l}356.5 \\
421.6 \\
340.9\end{array}$ & $\begin{array}{l}+637 \\
+751 \\
+540\end{array}$ & $\begin{array}{l}3.71 \\
3.38 \\
3.70\end{array}$ \\
\hline & $\begin{array}{l}2 \mathrm{~F} \\
2{ }^{2} \mathrm{D}^{\prime} \\
{ }^{2} \mathrm{P}\end{array}$ & $\begin{array}{l}w^{2} \mathrm{~F}_{4} \\
w^{2} \mathrm{D}^{\prime}{ }_{3} \\
w^{2} \mathrm{P}_{2}\end{array}$ & $\begin{array}{l}37,619.8 \\
36,618.5 \\
37,243.7\end{array}$ & $\begin{array}{l}206.9 \\
156.2 \\
-35.6\end{array}$ & $\begin{array}{r}(?) \\
-421 \\
+157\end{array}$ & $\begin{array}{r}(?) \\
(1.57) \\
(0.91)\end{array}$ \\
\hline & $\begin{array}{l}{ }^{2} \mathrm{P}^{\prime} \\
{ }^{2} \mathrm{~S}^{\prime}\end{array}$ & $\begin{array}{l}v^{2} \mathrm{D}^{\prime}{ }_{3} \\
z^{2} \mathrm{~S}_{1}^{\prime}\end{array}$ & $\begin{array}{l}40,572.4 \\
34,438.2\end{array}$ & 36.1 & & \\
\hline & ${ }^{2} \mathrm{P}$ & & & & & \\
\hline$(5 s)^{2} .6 s$ & ${ }^{2} \mathrm{~S}_{1}$ & $a^{2} \mathrm{~S}_{1}$ & $31,671.6$ & & & \\
\hline$(5 s)^{2} .7 \mathrm{~s}$ & ${ }^{2} \mathrm{~S}_{1}$ & $c^{2} \mathrm{~S}_{1}$ & $43,643.9$ & & & \\
\hline$(5 s)^{2} .5 d$ & ${ }^{2} \mathrm{D}$ & $e^{2} \mathrm{D}_{3}$ & $43,069.8$ & 414.0 & & \\
\hline$(5 s)^{2} .6 d$ & ${ }^{2} \mathrm{D}$ & & & & & \\
\hline \multirow[t]{2}{*}{$5 s .4 d .68$} & ${ }^{4} \mathrm{D}$ & $a^{4} \mathrm{D}_{4}$ & $33,752.7$ & $341.2,173.7,89.5$ & -815 & 3.42 \\
\hline & ${ }^{2} \mathbf{D}$ & $d^{2} \mathrm{D}_{3}$ & $36,431.0$ & 10.5 & +703 & (0.14) \\
\hline $58.4 d .7 s$ & ${ }^{4} \mathrm{D}$ & $c^{4} D_{4}$ & $44,922.1$ & 267.1 & & \\
\hline
\end{tabular}




\begin{tabular}{|c|c|c|c|c|c|c|}
\hline $\begin{array}{c}\text { Electron } \\
\text { configuration }\end{array}$ & $\begin{array}{l}\text { Predicted } \\
\text { terms }\end{array}$ & $\begin{array}{c}\text { Observed } \\
\text { terms }\end{array}$ & Main level & Level separations & Yt I-Sc I & $\begin{array}{c}\text { Ratio of } \\
\text { separations } \\
\text { Yt I/Sc I }\end{array}$ \\
\hline$(4 d)^{2} .6 s$ & $\begin{array}{l}{ }^{4 \mathrm{~F}^{\prime}} \\
{ }^{2} \mathrm{P}^{\prime} \\
{ }^{2} \mathrm{G} \\
{ }^{2} \mathrm{~F}^{\prime} \\
2 \mathrm{D} \\
{ }^{2} \mathrm{P}^{\prime} \\
{ }^{2} \mathrm{~S}\end{array}$ & $\begin{array}{l}e^{4} \mathrm{~F}^{\prime}{ }^{3} \\
? e^{4} \mathrm{P}^{\prime \prime}{ }_{3}\end{array}$ & $\begin{array}{l}44,759.6 \\
50,254.0\end{array}$ & $\begin{array}{l}393.9,312.7 ? ? \\
?, ?\end{array}$ & $+2,675$ & \\
\hline $5 s .4 d .5 d$ & $\begin{array}{l}{ }^{4} \mathrm{G} \\
{ }^{4} \mathrm{~F}^{\prime} \\
{ }^{4} \mathrm{D} \\
{ }^{4} \mathrm{P}^{\prime} \\
{ }^{4} \mathrm{~S} \\
\\
{ }^{2} \mathrm{G} \\
{ }^{2} \mathrm{~F}^{\prime} \\
{ }^{2} \mathrm{D} \\
{ }^{2} \mathrm{P}^{\prime} \\
{ }^{2} \mathrm{~S} \\
{ }^{2} \mathrm{G} \\
{ }^{2} \mathrm{~F}^{\prime} \\
{ }^{2} \mathrm{D} \\
{ }^{2} \mathrm{P}^{\prime}\end{array}$ & $\begin{array}{c}a^{4} \mathrm{G}_{6} \\
c^{4} \mathrm{~F}^{\prime}{ }_{5} \\
b^{4} \mathrm{D}_{4} \\
d^{4} \mathrm{P}_{3}^{\prime} \\
.\end{array}$ & $\begin{array}{l}39,233.0 \\
39,963.8 \\
38,865.6 \\
40,517.1\end{array}$ & $\begin{array}{l}283.7,187.3,126.4 \\
206.0,192.8,118.7 \\
190.2,131.7,73.8 \\
62.0, ?\end{array}$ & $\begin{array}{l}-795 \\
-707 \\
-924\end{array}$ & $\begin{array}{l}3.58 \\
3.46 \\
4.01 \\
\end{array}$ \\
\hline \multirow[t]{2}{*}{ 5s. $(5 p)^{2}$} & ${ }^{4} \mathrm{P}^{\prime}$ & $c^{4} \mathrm{P}_{3}^{\prime}$ & $34,521.2$ & $365.4,244.3$ & & \\
\hline & $\begin{array}{l}{ }_{2}^{2} \mathrm{D} \\
{ }_{2}^{2} \mathrm{P}^{\prime}\end{array}$ & $\begin{array}{l}?^{2} c^{2} \mathrm{D}_{3} \\
? b^{2} \mathrm{P}^{\prime} \\
? b^{2} \mathrm{~S}_{1}\end{array}$ & $\begin{array}{l}34,257.4 \\
33,842.3 \\
42,685.5\end{array}$ & $\begin{array}{r}26.2 \\
229.1\end{array}$ & & \\
\hline \multirow[t]{2}{*}{$4 d .(5 p)^{2}$} & $\begin{array}{l}{ }^{4} \mathrm{~F}^{\prime} \\
{ }^{4} \mathrm{D} \\
{ }^{\prime} \mathrm{P}^{\prime}\end{array}$ & $\begin{array}{l}f^{4} \mathrm{~F}^{\prime} s_{3} \\
d^{4} \mathrm{D}_{4}\end{array}$ & $\begin{array}{l}45,796.5 \\
45,204.0\end{array}$ & $\begin{array}{l}408.0,319.2,326.7 ? \\
195.7,260.0, \quad 88.1\end{array}$ & +673 & 3. 48 \\
\hline & 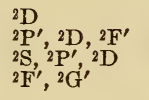 & $? c^{2} P^{\prime} 2$ & $45,994.0$ & 46.5 & & \\
\hline$(4 d)^{2} .5 d$ & $\begin{array}{l}{ }^{4} \mathrm{P}^{\prime},{ }^{4} \mathrm{D},{ }^{4} \mathrm{C}^{\prime} \\
{ }^{4} \mathrm{G},{ }^{4} \mathrm{H}^{\prime},{ }^{4} \mathrm{P}^{\prime} \\
{ }^{4} \mathrm{D},{ }^{4} \mathrm{~F}^{\prime} \\
\\
{ }^{2} \mathrm{D},{ }^{2} \mathrm{~F}^{\prime},{ }^{2} \mathrm{G} \\
{ }^{2} \mathrm{H}^{\prime},{ }^{2 \mathrm{I}},{ }^{2} \mathrm{P}^{\prime} \\
{ }^{2} \mathrm{D},{ }^{2} \mathrm{~F}^{\prime},{ }^{2} \mathrm{G} \\
{ }^{2} \mathrm{H}^{2}{ }^{2 \mathrm{~S}},{ }^{2 \mathrm{P}^{\prime}} \\
{ }^{2} \mathrm{D},{ }^{2} \mathrm{~F}^{2},{ }^{2} \mathrm{G} \\
{ }^{2} \mathrm{D},{ }^{2} \mathrm{~F}^{\prime}\end{array}$ & $? d^{4} \mathrm{~F}^{\prime} 8$ & $44,190.5$ & $486.1,366.9,241.8$ & & \\
\hline
\end{tabular}

Among the high quartet terms those from the configurations $d s .6 s$ and $d s .5 d$ are clearly recognizable by their strong combinations with the $d s p$ triad. The terms arising from $d s .7 s$ and $d^{2} .6 s$ have been identified by series relations; they give very faint lines, and some of the component levels have not been detected. The high term $d^{2} \mathrm{~F}^{\prime}$, which combines with the $d^{2} p$ terms to give fairly strong lines in the deep red may be assigned to $d^{2} .5 d$, though it lies lower than might have been expected. The other terms of this pentad probably give lines in the infra-red. The only other configurations which can give terms near the observed level are $s p^{2}$ and $d p^{2}$. The former gives ${ }^{4} \mathrm{P}^{\prime}$ which should be relatively low since the configuration contains no $d$ electron. This fits $c^{4} \mathrm{P}^{\prime}$. The latter gives ${ }^{4} \mathrm{~F}^{\prime},{ }^{4} \mathrm{D},{ }^{4} \mathrm{P}^{\prime}$, and $f^{4} \mathrm{~F}^{\prime}$ and $d^{4} \mathrm{D}$ may be placed here. All the quartet terms are now identified. 
The higher terms of the doublet system are much less fully developed (as usually happens with the lower multiplicity). The term $d^{2} \mathrm{D}$, which combines with the $d s p$ triad, may be assigned to $d s .6 s$; its separation is much smaller than should be expected, but the combinations appear to assure this interpretation. The configuration $d s .5 d$ probably gives lines just outside the limit of observation in the red. The remaining terms combine most strongly with $z^{2} \mathrm{P}$; three of them, $a^{2} \mathrm{~S}, b^{2} \mathrm{P}^{\prime}, c^{2} \mathrm{D}$, are confirmed by Zeeman patterns. The high term $c^{2} \mathrm{P}^{\prime}$ combines strongly with $z^{2} \mathrm{D}^{\prime}(d s p)$. The remaining levels, though undoubtedly real, are close enough together to make it difficult to arrange them into terms. The isolated lines $3252.28 \mathrm{~A}$, 3152.67 A and 3091.70 A give evidence by their behavior in the furnace that they originate from the $z^{2} \mathrm{P}$ level, but, in the absence of Zeeman effects, they can not be certainly classified.

The configurations which should combine strongly with $s^{2} p$ are $s^{2} \cdot s$, giving ${ }^{2} \mathrm{~S}, s^{2} d\left({ }^{2} \mathrm{D}\right)$, and $s p^{2}\left({ }^{2} \mathrm{P}^{\prime},{ }^{2} \mathrm{D},{ }^{2} \mathrm{~S}\right)$. The term $a^{2} \mathrm{~S}$ may be assigned with confidence to $s^{2} \cdot 6 s$. The level here called $c^{2} \mathrm{~S}$ may be in series with it though the corresponding lines are stronger than might be anticipated, and are inverted in intensity.

The assignment of the remaining levels is not so easy. There are two ${ }^{2} \mathrm{P}^{\prime}$ terms where only one is to be anticipated. The second must come from $d p^{2}$. A "double jump" is involved, but this sometimes gives strong lines. The $d p^{2}$ and $s p^{2}$ configurations should give stronger combinations with $d s p$ than $s^{2} s$ or $s^{2} d$, thus indicating that $c^{2} \mathrm{D}$ and $c^{2} \mathrm{P}^{\prime}$ come from these configurations. The latter lies surprisingly high, but the ${ }^{1} \mathrm{P}(s p)$ term in Yt II is also high. On the other hand, one would not expect a term from $d p^{2}$ to lie nearly as low as $b^{2} \mathrm{P}^{\prime}$. The lower term has, therefore, been provisionally assigned to $s p^{2}$, and the higher to $d p^{2}$. The other levels have been tentatively arranged into the terms $e^{2} \mathrm{D}, b^{2} \mathrm{~S}$, and $c^{2} \mathrm{~S}$, and assigned to $s^{2} d, s p^{2}$, and $s^{2} .7 s$; but these assignments are subject to correction when Zeeman data for the particular lines in question become available.

The intensities of the various combinations are shown in summary fashion in Table 11, which gives the values on Meggers's scale (except those in parenthesis) for the strongest line in each multiplet. The letter $a$ denotes that the multiplet is absent, $i$, that it lies in the infra-red, and $u$ that it is in the ultra-violet. The large number of predicted infra-red lines is noteworthy; some of them should be very strong. 
TABLE 11.-Intensities of strongest lines in Yt I muliplets

QUARTET SYSTEM

\begin{tabular}{|c|c|c|c|c|c|c|c|c|c|c|}
\hline & & \multicolumn{3}{|c|}{$d s p$} & \multicolumn{6}{|c|}{$d^{2} p$} \\
\hline & & $z^{4} \mathrm{~F}$ & $z^{4} \mathrm{D}^{\prime}$ & $z^{4} \mathrm{P}$ & $z^{4} \mathrm{G}^{\prime}$ & $y^{4} F$ & $y^{4} \mathrm{D}^{\prime}$ & $x^{4} \mathrm{D}^{\prime}$ & $\eta^{4} \mathrm{P}$ & $z^{4} \mathrm{~S}^{\prime}$ \\
\hline$d^{2} s$ & $\begin{array}{l}a^{4} \mathrm{~F}^{2} \\
a^{4} \mathrm{P}^{\prime}\end{array}$ & $\mathrm{i}$ & $\mathbf{i}$ & $\mathrm{i}$ & 50 & 60 & $\begin{array}{r}40 \\
2\end{array}$ & $\begin{array}{r}a \\
10\end{array}$ & 6 & 2 \\
\hline$d^{s}$ & $\begin{array}{l}b^{4} \mathrm{~F}^{\prime} \mathrm{F}^{4} \mathrm{P}^{\prime} \mathrm{P}^{\prime}\end{array}$ & 10 & $\begin{array}{l}7 \\
\mathrm{a}\end{array}$ & 10 & $\mathbf{i}$ & $\mathbf{i}$ & $\stackrel{i}{i}$ & $\begin{array}{l}\mathbf{i} \\
\mathbf{i}\end{array}$ & $\mathbf{i}$ & $\mathbf{i}$ \\
\hline $\begin{array}{l}d s .6 s \\
d s .78\end{array}$ & $\begin{array}{l}a^{4} \mathrm{D} \\
c^{4} \mathrm{D}\end{array}$ & $\begin{array}{r}15 \\
2\end{array}$ & $\begin{array}{l}8 \\
1\end{array}$ & $\begin{array}{r}5 \\
\mathrm{a}\end{array}$ & 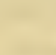 & i & $\begin{array}{l}\mathbf{i} \\
\mathbf{a}\end{array}$ & $\begin{array}{l}i \\
g\end{array}$ & $\begin{array}{l}\mathbf{i} \\
\mathbf{a}\end{array}$ & \\
\hline$d s .5 d$ & $\begin{array}{l}a^{4} \mathrm{G}^{4} \\
c^{4} \mathrm{~F}^{\prime} \\
b^{4} \mathrm{D} \\
d^{4} \mathrm{P}^{\prime}\end{array}$ & $\begin{array}{r}25 \\
5 \\
2\end{array}$ & $\begin{array}{r}1 \\
6 \\
10 \\
2\end{array}$ & $\begin{array}{l}2 \\
2\end{array}$ & $\stackrel{i}{\mathbf{i}}$ & $\begin{array}{l}\mathbf{i} \\
\mathbf{i} \\
\mathbf{i}\end{array}$ & $\begin{array}{l}\mathbf{i} \\
\mathbf{i} \\
\mathbf{i}\end{array}$ & $\begin{array}{l}\mathbf{i} \\
\mathbf{i} \\
\mathbf{i}\end{array}$ & $\begin{array}{l}\mathbf{i} \\
\mathbf{i}\end{array}$ & i \\
\hline$d^{2} . \hat{c} s$ & $\begin{array}{l}e^{4} \mathrm{~F}^{\prime} \\
e^{4} \mathrm{P}^{\prime}\end{array}$ & a & $\begin{array}{l}\mathrm{a} \\
\mathrm{a}\end{array}$ & a & 2 & 3 & $\begin{array}{l}a \\
a\end{array}$ & $\underset{2}{\mathrm{a}}$ & 1 & a \\
\hline$d^{2} .5 d$ & $d^{4} \mathrm{~F}^{\prime}$ & 5 & 3 & & 1 & 5 & $\mathbf{i}$ & a & 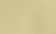 & . \\
\hline$s p^{2}$ & $c^{4} \mathrm{P}^{\prime}$ & & 6 & 0 & & & $\mathbf{i}$ & i & $\mathbf{i}$ & $\mathrm{i}$ \\
\hline$d p^{2}$ & $\int_{d^{*} D}^{4} F^{\prime}$ & $\stackrel{a}{2}$ & $\begin{array}{l}4 \\
a\end{array}$ & (2) & $\mathrm{u}$ & $\begin{array}{l}\mathrm{u} \\
\mathrm{u}\end{array}$ & $\mathrm{u}$ & $\begin{array}{l}\mathrm{a} \\
\mathrm{a}\end{array}$ & $\mathbf{u}$ & \\
\hline
\end{tabular}

DOUBLET SYSTEM

\begin{tabular}{|c|c|c|c|c|c|c|c|c|c|c|c|c|c|c|}
\hline \multirow{2}{*}{, } & & $d s^{2}$ & \multicolumn{4}{|c|}{$d^{2} s$} & \multirow{2}{*}{$\frac{d s .68}{d^{2} \mathrm{D}}$} & \multirow{2}{*}{$\frac{s^{2} \cdot 6 s}{a^{2} \mathrm{~S}}$} & \multirow{2}{*}{$\frac{s^{2} .7 \mathrm{~s}}{c^{2} \mathrm{~S}}$} & \multirow{2}{*}{$\begin{array}{l}s^{2} .5 d \\
e^{2} \mathrm{D} ?\end{array}$} & \multicolumn{3}{|c|}{$s p^{2}$} & \multirow{2}{*}{$\frac{d p^{2}}{c^{2} P^{\prime}}$} \\
\hline & & $a^{2} \mathrm{D}$ & $a^{2} \mathrm{G}$ & $a^{2} F^{\prime}$ & $b^{2} \mathrm{D}$ & $a^{2} \mathrm{P}^{\prime}$ & & & & & $b^{2} \mathrm{P}^{\prime}$ & $c^{2} \mathrm{D}$ & $b^{2} \mathrm{~S} ?$ & \\
\hline$s^{2} p$ & $z^{2} \mathrm{P}$ & 10 & & & $\mathbf{i}$ & i & 2 & 10 & 2 & (2) & 8 & 5 & 2 & 2 \\
\hline & $\begin{array}{c}z^{2} F \\
z^{2} \mathrm{D}^{\prime} \\
y^{2} \mathrm{P}\end{array}$ & $\begin{array}{r}50 \\
100 \\
40\end{array}$ & i & $\stackrel{i}{\mathbf{i}}$ & $\begin{array}{l}\mathbf{i} \\
\mathbf{i} \\
\mathbf{i}\end{array}$ & $\begin{array}{l}\mathbf{i} \\
\mathbf{i} \\
\mathbf{i}\end{array}$ & $\begin{array}{l}4 \\
3 \\
4\end{array}$ & $\mathbf{i}$ & & & $i_{i}^{2}$ & $\begin{array}{l}2 \\
2 \\
i\end{array}$ & $\mathbf{i}$ & $\begin{array}{c}3 \\
a\end{array}$ \\
\hline$d s p$ & $\begin{array}{c}y^{2} \mathrm{~F} \\
y^{2} \mathrm{D}^{\prime} \\
x^{2} \mathrm{P}\end{array}$ & $\begin{array}{r}150 \\
150 \\
50\end{array}$ & i & $\stackrel{i}{\mathbf{i}}$ & $\begin{array}{c}\mathrm{i} \\
\mathrm{i} \\
10\end{array}$ & $\begin{array}{l}\mathbf{i} \\
\mathbf{i} \\
\mathbf{i}\end{array}$ & $\begin{array}{l}\mathrm{i} \\
3 \\
\mathrm{i}\end{array}$ & $\mathbf{i}$ & & & $\underset{\mathbf{i}}{\mathbf{i}}$ & $\begin{array}{l}\mathbf{i} \\
\mathbf{i}\end{array}$ & $\mathrm{a}$ & $\begin{array}{l}a \\
a\end{array}$ \\
\hline & $\begin{array}{c}z^{2} \mathrm{H} \\
y^{2} \mathrm{G}^{\prime} \\
v^{2} \mathrm{~F}\end{array}$ & 3 & $\begin{array}{l}4 \\
6 \\
3\end{array}$ & $\begin{array}{c}\text { (2) } \\
\text { a }\end{array}$ & $3 ?$ & & & & & & & & & \\
\hline$d^{2} p$ & $\begin{array}{c}z^{2} G^{\prime} \\
x^{2} F \\
x^{2} D^{\prime}\end{array}$ & $\begin{array}{r}1 \\
10 \\
5\end{array}$ & $\begin{array}{r}15 \\
3\end{array}$ & $\begin{array}{l}10 \\
10 \\
20\end{array}$ & $\begin{array}{l}1 \\
2 \\
2\end{array}$ & 5 & & & & & & & & \\
\hline & $\begin{array}{c}w^{2} \mathrm{~F} \\
w^{2} \mathrm{D}^{\prime} \\
w^{2} \mathrm{P}\end{array}$ & $\begin{array}{l}\text { (1) } \\
3 \\
\text { (3) }\end{array}$ & 2 & $\frac{2}{3}$ & $\begin{array}{r}10 \\
3 \\
6\end{array}$ & & & & & & & & & \\
\hline & $\begin{array}{l}z^{2} \mathrm{~S}^{\prime} \\
?^{2} \mathrm{P} \\
v^{2} \mathrm{D} \mathrm{D}^{\prime}\end{array}$ & (1) & & 2 & a & 5 & & & & & & & & \\
\hline
\end{tabular}

Table 10 shows the predicted and observed terms and a comparison with the levels and separations of the corresponding terms in scandium. The arc spectra of the two elements are almost as much alike as the others. The levels are nearly the same except that the $a^{3}$ and $s p$ configurations lie lower in yttrium. The ratio of the separations is less regular than in the spark spectra, especially for some of the terms which go to singlet terms in the spark as limits. 
If the ratios given in parenthesis in Table 10 are excluded, the mean for the rest is 3.74 -nearly the same as for the higherstates of ionization.

\section{IONIZATION POTENTIALS OF YTTRIUM ATOMS}

The first two members of series have been identified in the spectrum of yttrium in all three degrees of ionization, and the ionization potentials for three different yttrium atoms, $\mathrm{Yt}, \mathrm{Yt}^{+}, \mathrm{Yt}^{++}$, may thus be determined.

1. For Yt III the application of a simple Rydberg formula to the $5^{2} \mathrm{~S}$ and $6^{2} \mathrm{~S}$ terms gives an ionization potential of 20.79 volts for the normal state $4^{2} \mathrm{D}_{2}$. Bowen and Millikan's value of 20.41 volts, which is derived from the value of the $4 \mathrm{~F}$ term estimated by comparison with $\mathrm{Rb} \mathrm{I}$ and $\mathrm{Sr}$ II, should be much more accurate. If the result of the Rydberg formula is corrected by the empirical method suggested by one of us, ${ }^{21}$ it becomes 20.63 volts-still too high. There is other evidence that this correction which holds good for the first long period of elements should roughly be doubled for the second. A Rydberg formula applied to the ${ }^{4} \mathrm{D}$ and ${ }^{5} \mathrm{D}$ terms gives 21.79 voltsmuch too high, as usual.

2. In Yt II, according to the analysis already made, the terms $b^{3} \mathrm{D}, c^{1} \mathrm{D}$, and $c^{1} \mathrm{~S}$ are in series with the low terms $a^{3} \mathrm{D}, a^{1} \mathrm{D}$, and $a^{1} \mathrm{~S}$, respectively, since they result from the latter when an electron is shifted from a $5 s$ to a $6 s$ state. Applying the Rydberg formula to the various components of these terms, we find the term values given below. These represent the interval between the term component in question and the limit of the series to which each belongs. In order to find the position of the series limit relative to the lowest energy state of $\mathrm{Yt}^{+}$, the observed term values relative to this state must be added. According to Hund's theory, ${ }^{3} \mathrm{D}_{3}$ and ${ }^{1} \mathrm{D}_{2}$ converge to ${ }^{2} \mathrm{D}_{3}$ in Yt III as a limit, and ${ }^{3} \mathrm{D}_{2}$ and ${ }^{3} \mathrm{D}_{1}$ to ${ }^{2} \mathrm{D}_{2}$. To find the difference between the lowest energy levels of the singly and doubly ionized atom, we must, therefore, subtract the difference ${ }^{2} \mathrm{D}_{3}-{ }^{2} \mathrm{D}_{2}=725$, from the sum found in the first two cases and ${ }^{2} \mathrm{~S}_{1}-{ }^{2} \mathrm{D}_{2}=7,466$ from the limit of the ${ }^{1} \mathrm{~S}$ series.

\begin{tabular}{|c|c|c|c|c|c|}
\hline & ${ }^{8} \mathrm{D}_{3}$ & ${ }^{3} \mathrm{D}_{2}$ & ${ }^{8} \mathrm{D}_{1}$ & 1D, & ${ }^{1} \mathrm{~S}_{0}$ \\
\hline Levels above ${ }^{1} \mathrm{~S}_{0}(\mathrm{Yt}$ II $)\left\{\begin{array}{l}(5 s) \ldots \\
(68)\end{array}\right.$ & $\begin{array}{r}1,450 \\
55,645\end{array}$ & $\begin{array}{r}1,045 \\
55,032\end{array}$ & $\begin{array}{r}840 \\
54,956\end{array}$ & $\begin{array}{r}3,296 \\
55,725\end{array}$ & $\begin{array}{r}0 \\
61,367\end{array}$ \\
\hline Distance to series limit $\left\{\begin{array}{l}(5 s) \\
(6 s)\end{array}\right.$ & $\begin{array}{r}100,015 \\
45,820\end{array}$ & $\begin{array}{l}99,711 \\
45,724\end{array}$ & $\begin{array}{l}99,902 \\
55,786\end{array}$ & $\begin{array}{l}97,443 \\
45,014\end{array}$ & $\begin{array}{r}110,300 \\
48,933\end{array}$ \\
\hline $\begin{array}{l}\text { Limit above }{ }^{1} \mathrm{~S}_{0} \\
\text { Limit above }{ }^{2 D_{2}}(\mathrm{Y} t \mathrm{i} \overline{\mathrm{I}})\end{array}$ & $\begin{array}{r}101,465 \\
725\end{array}$ & $\begin{array}{r}100,756 \\
0\end{array}$ & $\begin{array}{r}100,742 \\
0\end{array}$ & $\begin{array}{r}100,739 \\
725\end{array}$ & $\begin{array}{r}110,300 \\
7,466\end{array}$ \\
\hline${ }^{2} \mathrm{D}_{2}-1 \mathrm{~S}_{0}$ & 100,740 & 100,756 & 100,742 & 100,014 & 102,833 \\
\hline
\end{tabular}

21 Russell and Lang, Astrophys. J., 66, p. 247; 1927. 
The five determinations agree excellently. In particular, the distribution of the components of the ${ }^{3} \mathrm{D}$ term between the limits evidently agrees with Hund's predictions. The mean of the five determinations is 101,017 , corresponding to an ionization potential of 12.47 volts. Applying the doubled empirical correction mentioned above, this is reduced to 98,720 (12.11 volts). The round number 100,000 , or an ionization potential of 12.3 volts, is probably very near the truth.

3. In Yt I, $d^{2} \mathrm{D}$ is in series with $a^{2} \mathrm{D}$ and $d^{4} \mathrm{D}$ with $a^{4} \mathrm{D}$ (both converging to $a^{3} \mathrm{D}$ in $\mathrm{Yt} \mathrm{II);} e^{4} \mathrm{~F}^{\prime}$ with $a^{4} \mathrm{~F}^{\prime}$ (limit $a^{3} \mathrm{~F}^{\prime}$ ); $e^{4} \mathrm{P}^{\prime}$ with $a^{4} \mathrm{P}^{\prime}$ (limit $a^{3} \mathrm{P}^{\prime}$ ); and $c^{2} \mathrm{~S}$ with $a^{2} \mathrm{~S}$ (linnit $a^{1} \mathrm{~S}$ ). Treating the components of these terms as before, we find-

\begin{tabular}{|c|c|c|c|c|c|c|c|c|c|}
\hline & ${ }^{2} \mathrm{D}_{3}$ & ${ }^{2} \mathrm{D}_{2}$ & ${ }^{4} \mathrm{D}_{4}$ & ${ }^{4} \mathrm{D}_{3}$ & ${ }^{4} \mathrm{~F}_{6}^{\prime}$ & ${ }_{4}^{4} F_{4}^{\prime}$ & ${ }^{4} \mathrm{~F}_{2}$ & ${ }^{4} \mathrm{P}_{3}^{\prime}$ & ${ }^{2} \mathrm{~S}_{1}$ \\
\hline Levels above ${ }^{2} \mathrm{D}_{2}(\mathrm{Yt} \mathrm{I})$ & $\left\{\begin{array}{r}530 \\
36,431\end{array}\right.$ & $\begin{array}{r}0 \\
36,421\end{array}$ & $\begin{array}{l}33,752 \\
44,922\end{array}$ & $\begin{array}{l}33,411 \\
44,655\end{array}$ & $\begin{array}{l}11,532 \\
44,760\end{array}$ & $\begin{array}{l}11,278 \\
44,366\end{array}$ & $\begin{array}{l}11,079 \\
44,053\end{array}$ & $\begin{array}{l}15,477 \\
50,254\end{array}$ & $\begin{array}{l}31,672 \\
43,643\end{array}$ \\
\hline Distances to limit_. & $\left\{\begin{array}{l}54,690 \\
18,789\end{array}\right.$ & $\begin{array}{l}55,340 \\
18,929\end{array}$ & $\begin{array}{l}21,498 \\
10,328\end{array}$ & $\begin{array}{l}21,610 \\
10,366\end{array}$ & $\begin{array}{l}51,325 \\
18,097\end{array}$ & $\begin{array}{l}51,148 \\
18,060\end{array}$ & $\begin{array}{l}51,079 \\
18,045\end{array}$ & $\begin{array}{l}53,278 \\
18,501\end{array}$ & $\begin{array}{l}22,692 \\
10,721\end{array}$ \\
\hline Limit above ${ }^{2} \mathrm{D}_{2}$ & $\left\{\begin{array}{l}55,220 \\
1,450\end{array}\right.$ & $\begin{array}{r}55,340 \\
1,045\end{array}$ & $\begin{array}{r}55,250 \\
1,450\end{array}$ & $\begin{array}{r}55,021 \\
1,045\end{array}$ & $\begin{array}{r}62,857 \\
8,743\end{array}$ & $\begin{array}{r}62,426 \\
8,328\end{array}$ & $\begin{array}{r}62,158 \\
8,003\end{array}$ & $\begin{array}{l}68,755 \\
14,098\end{array}$ & $\begin{array}{r}54,364 \\
0\end{array}$ \\
\hline$a^{1} \mathrm{~S}_{0}-a^{2} \mathrm{D}_{2-}$ & 53,770 & 54,295 & 53,800 & 53,976 & 54,114 & 54,098 & 54,155 & 54,657 & $54 ; 364$ \\
\hline
\end{tabular}

The general agreement is again good, though the two components of the ${ }^{2} \mathrm{D}$ term are rather discordant. The mean of the nine values is 54,127 , corresponding to an ionization potential of 6.68 volts. Applying the appropriate correction reduces this to 6.5 volts, and this is probably very close to the actual ionization potential of neutral yttrium atoms.

\section{YTTRIUM IN THE SUN}

The evidence for the existence of both neutral and ionized yttrium in the sun's atmosphere is conclusive, but the two sets of lines behave very differently.

The enhanced lines are well represented. Of those which arise (in absorption) from the low levels, $a^{1} \mathrm{~S}, a^{3} \mathrm{D}, a^{1} \mathrm{D}$, all but the faintest, including many intercombinations, are present or else masked by lines of other elements. The strongest lines reach the intensity 3 on Rowland's scale. The next level $a^{3} \mathrm{~F}^{\prime}$ gives lines of maximum intensity 2 ; the higher levels $a^{3} \mathrm{P}^{\prime}, b^{1} \mathrm{D}, a^{1} \mathrm{G}$, none exceeding intensity 0 , but all except the fainter lines are either present in the sun or masked. The lines which arise from transitions between the middle and higher terms and are faint in the arc are almost absent from the solar spectrum; the strongest of all, $z^{3} \mathrm{~F}_{4}-a^{3} \mathrm{G}_{5}$ (3173.07 A), shows no trace, but $z^{3} \mathrm{~F}_{4}-b^{3} \mathrm{D}_{3}(3668.50 \mathrm{~A})$ is faintly present.

In the spot spectrum the lines arising from the three lowest levels are very little affected, being, perhaps, slightly weakened. Those 
coming from $a^{3} \mathrm{~F}^{\prime}$ are decidedly weakened by a unit of Rowland's scale, and the faint lines from $a^{3} \mathrm{P}^{\prime}$ and $b^{1} \mathrm{D}$ are practically obliterated.

The arc lines are at best very faint in the spectrum of the sur's disk, but are strengthened to a remarkable degree in the spots. Of the multiplets arising from the low term $a^{2} \mathrm{D}$ the stronger lines are present or masked in the Fraunhofer spectrum, but the strongest of all, $a^{2} \mathrm{D}_{3}-y^{2} \mathrm{~F}_{4}(4102.38 \mathrm{~A}$ ), is only of intensity 0 . In the spot spectrum these lines are greatly strengthened, the change increasing from two of Rowland's units in the violet to about five in the red. Many of the fainter satellites and intercombinations which are absent from the ordinary solar spectrum appear in that of the spots.

For the next level $a^{4} \mathrm{~F}^{\prime}$ the strong "diagonal" lines of the two principal multiplets, when not masked by other lines, are very feebly' present (with maximum intensity-2) and are moderately strengthened in the spots by about one unit. The weaker lines are absent, even in the spots. About 20 other lines arising from this and from higher levels are attributed to yttrium in the solar spectrum tables. The low-temperature line 5556.43 A is strengthened in the spots, and may really belong to $\mathrm{Yt} \mathrm{I}$. Not one of the others is strengthened, and it is therefore practically certain that the solar lines are of other origin and the agreement in wave length with.yttrium lines is accidental.

The data on yttrium lines identified with solar spectrum lines may be summarized as follows:

\begin{tabular}{|c|c|c|c|c|c|}
\hline & Yt I & Yt II & & Yt I & Yt II \\
\hline $\begin{array}{l}\text { Present in spots only } \\
\text { Unblended } \\
\text { Blended with weaker lines }\end{array}$ & $\begin{array}{l}10 \\
10\end{array}$ & $\begin{array}{r}42 \\
7\end{array}$ & $\begin{array}{l}\text { Doubtful } \\
\text { Total } \\
\text { Not due to yttrium }\end{array}$ & $\begin{array}{r}4 \\
37 \\
22\end{array}$ & $\begin{array}{r}5 \\
72\end{array}$ \\
\hline Masked by other lines............. & 13 & 18 & & & \\
\hline
\end{tabular}

This behavior is exactly what should be expected of an element of moderate abundance and easy ionization. Over the disk almost all the atoms are ionized, and the arc lines are very faint. Over the spots the percentage of ionization, though diminished, is still large, so that the arc lines gain much more in proportion than the enhanced lines lose. The lowered temperature diminishes the relative number of atoms in excited states of higher energy, so that the lines absorbed by such atoms weaken, relatively to those arising from the normal state, for both the neutral and ionized atoms.

Washington and Princeton, October 25. 1928. 